\title{
Effect of Inlet Temperature Non-Uniformity on High-Pressure Turbine Performance
}

\author{
Craig I. Smith \\ Thesis submitted to the Faculty of Graduate and Postdoctoral Studies \\ in partial fulfillment of the requirements for the degree of

\section{MASTER OF APPLIED SCIENCE} \\ in Mechanical Engineering
Ottawa-Carleton Institute for Mechanical and Aerospace Engineering University of Ottawa
Ottawa, Canada

October 2010

(C) 2010 Craig I. Smith 


\begin{abstract}
The temperature of the flow entering a high-pressure turbine stage is inherently non-uniform, as it is produced by several discrete, azimuthally-distributed combustors. In general, however, industrial simulations assume inlet temperature uniformity to simplify the preparation process and reduce computation time. The effects of a non-uniform inlet field on the performance of a commercial, transonic, single-stage, high-pressure, axial turbine with a curved inlet duct have been investigated numerically by performing URANS (Unsteady Reynolds-Averaged Navier-Stokes equations) simulations with the SST (Shear Stress Transport) turbulence model. By adjusting the alignment of the experimentallybased inlet temperature field with respect to the stator vanes, two clocking configurations were generated: a vane-impinging (VI) case, in which each hot streak impinged on a vane; and a mid-pitch (MP) case, in which each hot streak passed between two vanes.

In the $V I$ configuration, the hot streaks produced higher time-averaged heat load on the vanes and lower heat load on the blades. As the hot streaks in the VI case passed over the stator vanes, they also spread spanwise due to the actions of the casing passage vortices and the radial pressure gradient; this resulted in a stream entering the rotor with relatively low temperature variations. The hot streaks in the $M P$ case were convected undisturbed past the relatively cool vane section. Relatively high time-averaged enthalpy values were found to occur on the pressure side of the blades in the $M P$ configuration. The non-uniformity of the time-averaged enthalpy on the blade surfaces was lower in the $V I$ configuration. The flow exiting the rotor section was much less non-uniform in the VI case, but differences in calculated efficiency were not significant.
\end{abstract}


for Claudine and Henri 


\section{Acknowledgments}

I would like to thank my supervisor, Dr. Stavros Tavoularis, for his guidance and assistance with this project, and for the opportunities that he's provided. Recognition goes out to Dr. Dongil Chang for his support and help that was available every day. This research could not have been completed without the financial support received from P\&WC, NSERC, the University of Ottawa, and HPCVL. Thanks goes out to them for their choice to invest in our project over the many others available.

A great amount of gratitude is owed to my family for their support throughout this research.

Finally, I cannot give enough thanks to my friends, both in and outside the university, for encouraging me and providing much needed stress relief. 


\section{Contents}

Nomenclature vii

List of Figures $\quad$ xiv

List of Tables $\quad$ xix

1 Introduction $\quad 1$

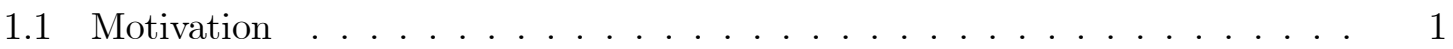

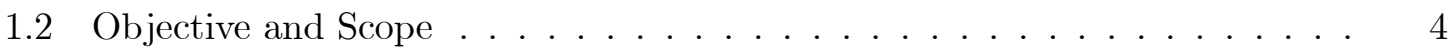

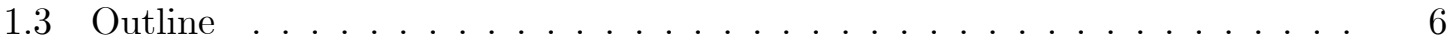

2 Literature Review $\quad 7$

2.1 Inlet Temperature Distribution . . . . . . . . . . . . . . . . . 8

2.2 Hot Streak Clocking . . . . . . . . . . . . . . . . . . . . . . . . 10

2.3 Buoyancy and Centrifugal Effects . . . . . . . . . . . . . . 11

2.4 Published Observations . . . . . . . . . . . . . . . . . . . 12

3 Background on Flows in High-Pressure Turbines $\quad 16$

3.1 Shocks . . . . . . . . . . . . . . . . . . . 17

3.2 Tip Leakage Flow . . . . . . . . . . . . . . . . . . . . . . . . . . . . . . . . . . . . . . . . .

3.3 Secondary Flows . . . . . . . . . . . . . . . . . . . . 21

3.4 Passage Vortices . . . . . . . . . . . . . . . . . . . 25

3.5 Effects of Temperature Nonuniformity on Flow Distribution . . . . . . . . . 27

3.5.1 Substitution Principle . . . . . . . . . . . . . . . 28

3.5.2 Positive and Negative Jets . . . . . . . . . . . . . . . . . 29

3.6 Qualification of Losses, Efficiency and Flow Coefficients . . . . . . . . . . 30

4 Numerical Background $\quad 37$

4.1 Governing Equations . . . . . . . . . . . . . . . . . . . 37

4.1.1 Unsteady Reynolds Averaged Navier-Stokes Equations . . . . . . . . 38

4.1.2 Favre Averaged Navier-Stokes Equations . . . . . . . . . . . . . . . 41

4.1 .3 Choice of CFD Code . . . . . . . . . . . . . . . . . . 42

4.2 Turbulence Modelling . . . . . . . . . . . . . . . . . . . 42 
4.2.1 Spalart-Allmaras (S-A) model . . . . . . . . . . . . . . . . 44

4.2 .2 Standard $k-\varepsilon$ model . . . . . . . . . . . . . . . . . . . 44

4.2 .3 RNG $k-\varepsilon$ model . . . . . . . . . . . . . . . . . . . . 45

4.2.4 Realizable $k-\varepsilon$ model . . . . . . . . . . . . . . . . . . 45

4.2.5 Shear Stress Transport (SST) model . . . . . . . . . . . . . . 46

4.2 .6 Reynolds Stress Model (RSM) . . . . . . . . . . . . . . . . . . 48

4.3 Discretization Schemes . . . . . . . . . . . . . . . . . . . 49

4.3.1 Grid Selection and Spatial Discretization _ . . . . . . . . . 49

4.3 .2 Temporal Discretization . . . . . . . . . . . . . . 54

4.4 Domain Scaling . . . . . . . . . . . . . . . . . . . 55

5 Inlet Temperature Specification $\quad \mathbf{5 7}$

5.1 Definition of Inlet Temperature Field . . . . . . . . . . . . . . . 57

5.1 .1 Spanwise Profile . . . . . . . . . . . . . . . . 58

5.1 .2 Circumferential Variation . . . . . . . . . . . . 59

5.2 Circumferential Positioning of Hot Streaks . . . . . . . . . . . . . . 63

5.3 Implementation . . . . . . . . . . . . . . . . . . . . . . 65

6 Numerical Procedures and Conditions $\quad 66$

6.1 Hardware and Software . . . . . . . . . . . . . . . 66

6.2 Computational Domain and Grid Generation . . . . . . . . . . . . 67

6.2 .1 Grid Verification . . . . . . . . . . . . . 71

6.3 Simulation Conditions . . . . . . . . . . . . . . . . . 72

6.4 Modelling Rotation . . . . . . . . . . . . . . . . . . . 77

6.4.1 Mixing Plane Model . . . . . . . . . . . . . . . . . . . 78

6.4.2 Sliding Mesh Model . . . . . . . . . . . . . . . . . . . . . 79

6.5 Simulation Methodology . . . . . . . . . . . . . . . . . . 80

6.6 Simulation Uncertainty . . . . . . . . . . . . . . . . . . . 83

7 Reference Simulation Results 90

7.1 Simulation Verification . . . . . . . . . . . . . . . . . . . . 91

7.1.1 Comparison Between Steady and Unsteady Simulations . . . . . . . 92

7.2 Coherent Vortices . . . . . . . . . . . . . . . . . . 97

7.2.1 Coherent Structure Identification . . . . . . . . . . . . . . . 98

7.2 .2 Stationary Vortices . . . . . . . . . . . . . . . . . . . . . 101

7.2 .3 Axial Gap Vortices . . . . . . . . . . . . . . . . . . . . . . . . . . . . . . . . . . . 105

7.2 .4 Crown Vortices . . . . . . . . . . . . . . . . . . . . . 110

7.3 Flow Field Characteristics . . . . . . . . . . . . . . . . . . 110

7.3.1 Shock Waves and Potential Fields . . . . . . . . . . . . . . 110

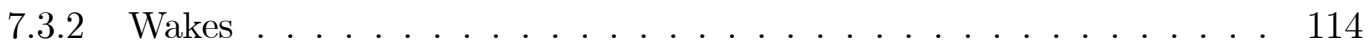

7.4 Summary . . . . . . . . . . . . . . . . . . . . . . . 119 
8 Hot Streak Simulation Results and Discussion $\quad 121$

8.1 In-Flow Property Variation . . . . . . . . . . . . . . . . . . . . . 121

8.2 Surface Property Variation _ . . . . . . . . . . . . . . . . . . . . 131

8.2 .1 Vane Surfaces . . . . . . . . . . . . . . . . . . . . . . 131

8.2 .2 Blade Surfaces . . . . . . . . . . . . . . . . . . . . . 133

8.3 Rotor Inlet and Outlet Properties . . . . . . . . . . . . . . . . . 135

8.4 Spatially-Averaged Properties . . . . . . . . . . . . . . . . 139

8.5 Turbine Efficiency . . . . . . . . . . . . . . . . . . . . 141

9 Conclusions and Recommendations for Future Work 143

9.1 Conclusions . . . . . . . . . . . . . . . . . . . . 143

9.2 Recommendations for Future Work . . . . . . . . . . . . . . . 145 


\section{Nomenclature}

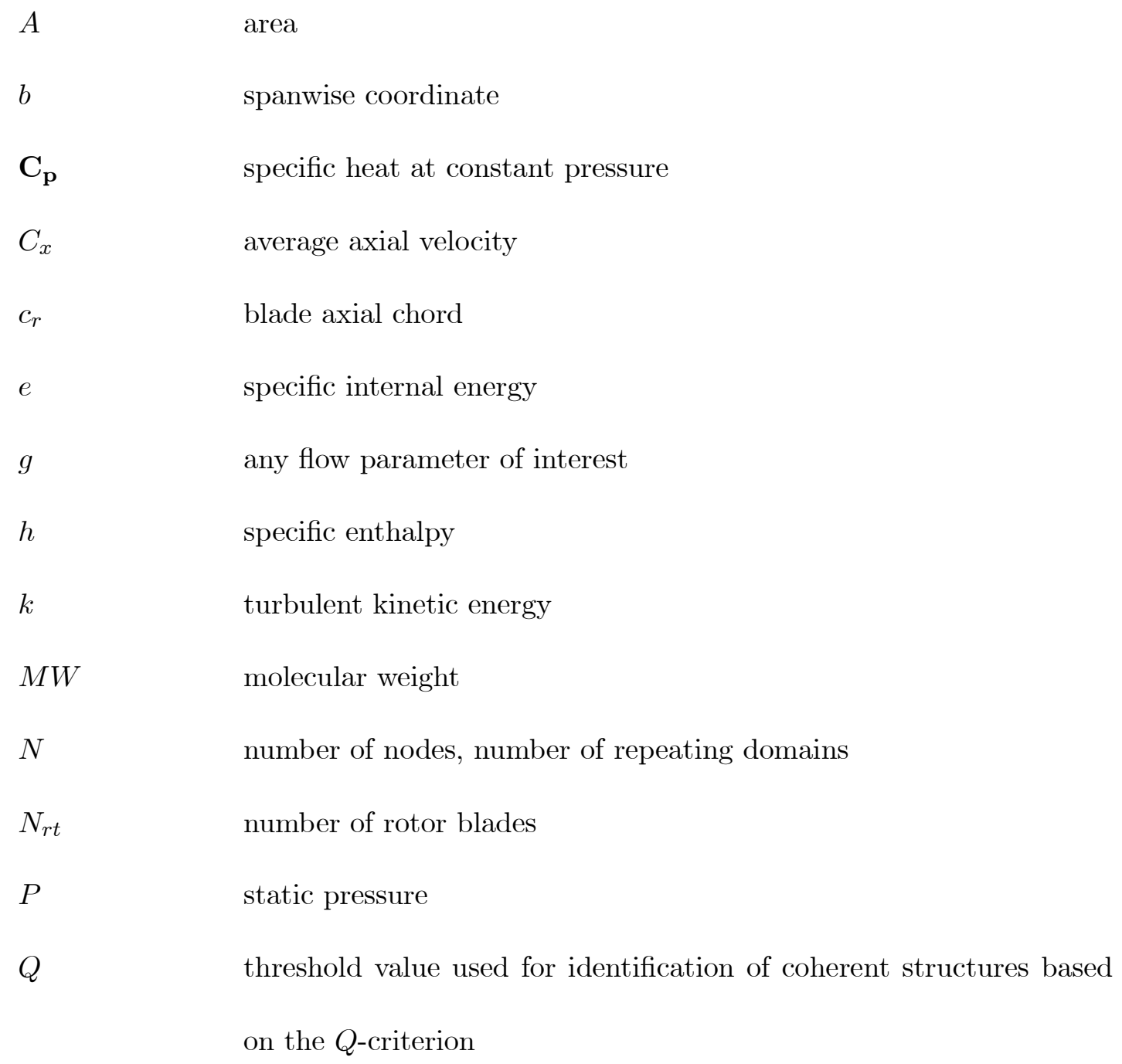




$\begin{array}{ll}Q_{m} & \text { threshold value used for identification of coherent structures based } \\ R & \text { on the modified } Q \text {-criterion } \\ \mathbf{R} & \text { local radius of curvature } \\ R e & \text { gas constant } \\ r & \text { Reynolds number } \\ s & \text { radial coordinate } \\ T & \text { specific entropy, streamwise coordinate } \\ t & \text { temperature } \\ U_{r} & \text { time } \\ Y_{p} & \text { velocity magnitude } \\ Y_{A}, v, w & \text { streamwise, crossflow/secondary flow, and spanwise velocity compo- } \\ u_{i}, u_{j}, u_{k} & \text { nents } \\ & \text { velocity vector in index notation } \\ & \end{array}$


Greek symbols

$\gamma$

$\Delta \quad$ difference in property

$\delta$

$\delta_{i j}$

$\epsilon_{i j k}$

$\varepsilon$

$\zeta_{h}$

$\zeta_{s}$

$\eta_{t t}$

$\eta_{t t i s}$

$\theta_{r p}$

$\kappa$

$\kappa_{e f f}$

$\kappa_{t}$

$\Lambda$

$\mu$

$\mu_{e f f}$

$\mu_{t}$

$\nu_{t}$

ratio of specific heats

kronecker delta

Levi-Civita tensor

kniematic viscosity relative change in a flow parameter

turbulent dissipation rate

enthalpy loss coefficient

entropy loss coefficient

total-to-total efficiency

total-to-total isentropic efficiency

angle of rotationally periodic sector of full turbomachine

coefficient of thermal conductivity of material

effective coefficient of thermal conductivity

coefficient of thermal conductivity due to turbulence

ratio of number of rotor blades to number of stator vanes in a stage

effective turbulent kinematic viscosity

turbulent kinematic viscosity

turbulent eddy viscosity 


$\begin{array}{ll}\rho & \text { density } \\ \tau_{r} & \text { rotor blade passing period } \\ \tau_{t} & \text { turbulent shear stress } \\ \Phi & \text { residual for a given flow parameter } \\ \phi & \text { flow coefficient } \\ \varphi & \text { circumferential coordinate } \\ \varphi_{0} & \text { circumferential phase shift } \\ \omega & \text { specific dissipation rate, rotational speed in Einstein notation } \\ \omega_{s}, \omega_{b}, \omega_{n} & \text { streamwise, spanwise, and normal components of vorticity }\end{array}$




\section{Subscripts}

$\begin{array}{ll}A, B & \text { associated with streamline } A A A \text { or } B B B \\ \text { av } & \text { average property } \\ i & \text { mass-weighted bulk property } \\ i, j, k & \text { iteration or index number } \\ m & \text { indices for Einstein notation } \\ r e l & \text { in the relative frame of reference } \\ s, b, n & \text { streamwise, spanwise and normal components } \\ t & \text { total property } \\ t i p & \text { associated with the blade tip } \\ 01 s, 02 s, 03 s & \text { inlet or farfield condition } \\ 1,2 s, 3 s & \text { total isentropic property at state } 1,2, \text { or } 3 \\ 01,02,03 & \text { property at state } 1,2, \text { or } 3 \\ & \text { total property at state } 1,2, \text { or } 3 \\ & \end{array}$




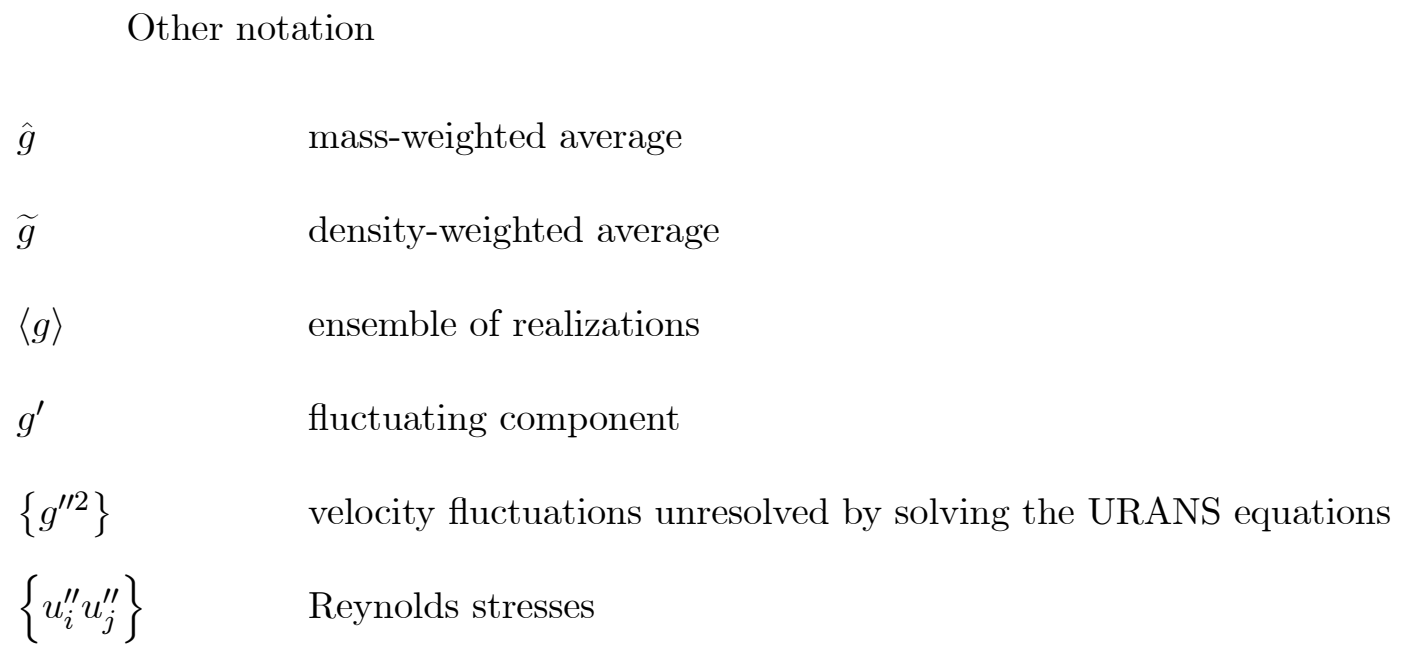




\begin{tabular}{|c|c|}
\hline GCI & grid convergence index \\
\hline HPCVL & High Performance Computing and Virtual Laboratory \\
\hline LE & leading edge \\
\hline LES & large eddy simulation \\
\hline MP & mid-pitch configuration \\
\hline $\mathrm{OCE}$ & overall convergence level \\
\hline $\mathrm{PS}$ & pressure side \\
\hline RANS & Reynolds averaged Navier-Stokes equations \\
\hline $\mathrm{RBC}$ & reflecting boundary condition \\
\hline RNG & renormalization group \\
\hline RSM & Reynolds stress model \\
\hline $\mathrm{S}-\mathrm{A}$ & Spalart-Allmaras \\
\hline SS & suction side \\
\hline $\mathrm{SST}$ & shear stress turbulent \\
\hline TE & trailing edge \\
\hline UDF & user-defined function \\
\hline UFANS & unsteady Favre averaged Navier-Stokes equations \\
\hline URANS & unsteady Reynolds averaged Navier-Stokes equations \\
\hline
\end{tabular}




\section{List of Figures}

1.1 Schematic diagram of a modern gas turbine blade with common cooling techniques $(\operatorname{Han}(2004)) . \ldots \ldots \ldots \ldots$

1.2 Section view of gas turbine portion of a similar turboshaft engine. The reverse-flow combustor has been indicated by (a), and the simulated highpressure stage has been indicated by (b). (Courtesy of the Aircraft Engine

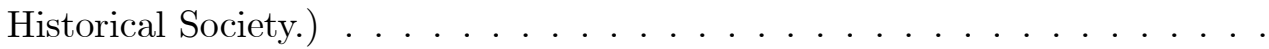

3.1 Structure of supersonic trailing edge shock system (Denton and Xu (1989)).

3.2 Schematic illustration of the development of secondary flow in a rotor blade passage (Lakshminarayana (1996)) . . . . . . . . . . . . . . . . . 22

3.3 Passage vortex model of Takeishi et al. (1990) . . . . . . . . . . . 26

3.4 Horseshoe vortex topology (Xun et al. (2005)). . . . . . . . . . . . 28

3.5 Enthalpy-entropy diagram for the expansion process in a single-stage turbine (based on Lakshminarayana (1996)). . . . . . . . . . . . . . . . . . . . . . 34

4.1 Example of a 2D geometry meshed using an H-type structured grid, from Lastiwka (2008). . . . . . . . . . . . . . . . . . . .

4.2 Two-dimensional example of an unstructured tetrahedral grid, from Lastiwka

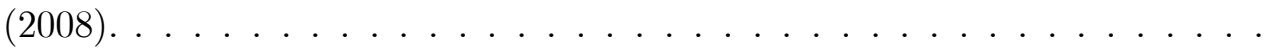

4.3 A hybrid mesh with structured cells near wall regions and unstructured cells elsewhere; from Lastiwka (2008). . . . . . . . . . . . . . . . . . .

5.1 Circumferentially-averaged spanwise profile of total temperature at the inlet, normalized by the mass-weighted bulk total temperature across the inlet $T_{b}$; the value of $T_{b}$ has been withheld according to the non-disclosure agreement with the industrial sponsor. . . . . . . . . . . . . 58

5.2 Typical radial profile of turbine inlet total temperature (Qingjun et al., 2007) 60

5.3 Original and extreme spanwise profiles of total temperature at the inlet, normalized by $T_{b} \ldots \ldots \ldots \ldots \ldots \ldots$

5.4 Contours of total temperature on the inlet, normalized by $T_{b}$. Dotted line indicates downstream position of vane leading edge. . . . . . . . . . 
5.5 Flow pathlines approaching stator vane. Arrow indicates adjusted location of aligned hot streak. . . . . . . . . . . . . . . . . .

5.6 Contours of inlet total temperature, normalized by $T_{b}$, for the (a) $V I$ and (b) $M P$ configurations. Dotted lines indicate downstream position of vane leading edge. . . . . . . . . . . . . . . . . .

6.1 Numerical domain, separated into three volumes; the stator and duct volumes are fixed, whereas the rotor volume is rotating. . . . . . . . . . 68

6.2 Surface mesh for computational grid. . . . . . . . . . . . . . . . 70

6.3 Boundary conditions in computational domain. . . . . . . . . . . . 75

6.4 Boundary conditions in computational domain. . . . . . . . . . . 75

6.5 Schematic diagram of a rotating domain (based on Ansys (2006a)) . . . . . 78

6.6 Schematic diagram of a sliding mesh interface (based on Ansys (2006a)). . . 80

6.7 Convergence levels for reference simulation . . . . . . . . . . . . . . 82

7.1 Flow parameter computed using three grids of decreasing cell size. Error bars show GCI for each grid, and dashed line indicates exact value as estimated by the AES method. . . . . . . . . . . . . . . . . .

7.2 Vortices in the rotor section, coloured by axial vorticity: (a) steady contours from RANS, (b) instantaneous contours from URANS. From Chang and Tavoularis $(2009 \mathrm{a}) . \ldots \ldots \ldots \ldots$

7.3 Contours of static pressure, normalized by $P_{o 1}$, at $50 \%$ span: (a) steady contours from RANS, (b) time-averaged contours from URANS. From Chang and Tavoularis (2009a). . . . . . . . . . . . . .

7.4 Contours of entropy, normalized by $\mathbf{R}$, at $50 \%$ span: (a) steady contours from RANS, (b) time-averaged contours from URANS. From Chang and Tavoularis $(2009 \mathrm{a}) \ldots \ldots \ldots \ldots \ldots \ldots$

7.5 Contours of relative total pressure loss coefficient at the rotor outlet plane: (a) steady contours from RANS, (b) time-averaged contours from URANS. View is from downstream of the rotor outlet plane. From Chang and Tavoularis

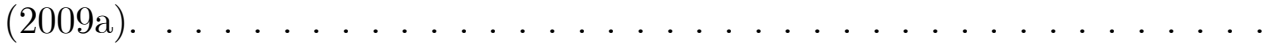

7.6 Contours of static pressure normalized by total inlet pressure: (a) steady contours from RANS, (b) time-averaged contours from URANS. From Chang and Tavoularis $(2009 \mathrm{a}) . \ldots \ldots \ldots \ldots \ldots$

7.7 Vortices identified by the modified $\mathrm{Q}$ criterion: (a) vortices in the stator (green), the rotor (red) and the extended duct (light blue); (b) top view of vortices in the rotor coloured by axial vorticity value; (c) left view of vortices in the rotor; (d) right view of vortices in the rotor. Vortex types: stator casing horseshoe (1), stator casing passage (2), stator trailing edge (3), rotor hub horseshoe (4), rotor hub passage (5), rotor casing horseshoe (6), rotor casing passage (7), rotor tip leakage (8), rotor trailing edge (9), rotor crown (10), axial gap (11). From Chang and Tavoularis (2009b). . . . . . . . . . . 102 
7.8 Effects of vortices on the suction side of rotor blade at $t / \tau_{r}=0.5$ : (a) vortices coloured by axial vorticity (red for counterclockwise rotation, blue for clockwise); (b) pathlines released from the blade surface (red lines from the blade tip, dark blue from the leading edge, light blue from the suction side, and yellow from the trailing edge of the blade; dashed black lines mark separation lines); (c) surface pressure isocontours, normalized by the total inlet pressure (d) wall shear stress. From Chang and Tavoularis (2009b). . .

7.9 Modified Q contours $10 \%$ of $c_{r}$ (blade axial chord) downstream of rotor trailing edge at (a) $t / \tau_{r}=0.0$, and (b) $t / \tau_{r}=0.5$. Identified cross-sections include that of the rotor hub passage vortex (5), the rotor casing passage vortex (7), and the tip leakage vortex (8). View is from downstream of the rotor section in the upstream direction. From Chang and Tavoularis (2009b). 106

7.10 Illustration showing the location of the axial gap vortex (11), remaining axially upstream of the leading edge of the rotor blade, (a) as the vortex forms at $t / \tau_{r}=0.0$, and (b) as it disappears at $t / \tau_{r}=0.5$ (see 7.11 for the relative vane/blade positions at these times). From Chang and Tavoularis (2009b).

7.11 Instantaneous entropy contours at $50 \%$ blade span; a white ellipse marks the location of the axial gap vortex; (a) $t / \tau_{r}=0.0$ (strong vortex), (b) $t / \tau_{r}=0.5$ (no vortex identified). From Chang and Tavoularis (2009b). . . . . . . . .

7.12 Contours of instantaneous static pressure, normalized by the total inlet pressure, at $50 \%$ blade span; a white ellipse marks the location of the axial gap vortex, whereas a hand-drawn dashed black line indicates a possible shock location; (a) $t / \tau_{r}=0.0$ (strong vortex), (b) $t / \tau_{r}=0.5$ (no vortex identified). From Chang and Tavoularis $(2009 \mathrm{~b}) \ldots \ldots \ldots \ldots$

7.13 Instantaneous contours of static pressure normalized by the total inlet pressure on blade surfaces and on two planes in the flow, at $50 \%$ and $10 \%$ span; (a) $t / \tau_{r}=0.0$, (b) $t / \tau_{r}=0.5$; dashed lines indicate possible vane shock locations. From Chang and Tavoularis (2009b). . . . . . . . . .

7.14 Instantaneous contours of static pressure normalized by the total inlet pressure on two planes in the flow, at $50 \%$ and $10 \%$ span; (a) $t / \tau_{r}=0.0$, (b) $t / \tau_{r}=0.5$; dashed lines indicate likely rotor shock locations. From Chang and Tavoularis (2009b). . . . . . . . . . . . . .

7.15 Instantaneous contours of relative Mach number on two planes in the flow, at $50 \%$ and $10 \%$ span, and at times (a) $t / \tau_{r}=0.0$, and (b) $t / \tau_{r}=0.5$; handdrawn dashed lines indicate possible rotor shock locations. From Chang and Tavoularis (2009b) . . . . . . . . . . . . . . .

7.16 Contours of static pressure normalized by the total inlet pressure on the stator outlet plane at times (a) $t / \tau_{r}=0.0$, and (b) $t / \tau_{r}=0.5$; hand-drawn dashed circles emphasize a local low-pressure region, dashed lines mark approximate locations of vane trailing edge shocks, and view is from downstream. From Chang and Tavoularis $(2009 b) . \ldots \ldots \ldots \ldots$ 
7.17 Contours of static pressure normalized by the total inlet pressure on a plane normal to the turbine axis at an axial location that is downstream of the rotor blade trailing edges by a distance equal to $10 \%$ of the axial blade chord, at times (a) $t / \tau_{r}=0.0$, and (b) $t / \tau_{r}=0.5$; view is from downstream and dashed lines mark possible blade trailing edge shocks. From Chang and Tavoularis

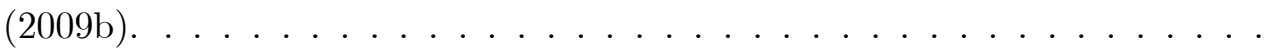

7.18 Instantaneous entropy contours on two planes in the flow, at $50 \%$ and $10 \%$ span and times (a) $t / \tau_{r}=0.0$, and (b) $t / \tau_{r}=0.5$. From Chang and Tavoularis $(2009 \mathrm{~b}) \ldots \ldots \ldots \ldots \ldots$

7.19 Contours of entropy on a plane normal to the turbine axis at an axial location that is downstream of the trailing edges of the rotor blades by a distance equal to $10 \%$ of the axial blade chord, at times (a) $t / \tau_{r}=0.0$, and (b) $t / \tau_{r}=0.5$; view is from downstream. From Chang and Tavoularis (2009b). . . . . . . .

8.1 Instantaneous contours of total enthalpy in a plane slightly upstream of the vane trailing edge for the (a) $V I$ and (b) $M P$ cases, normalized by $h_{o 1} \ldots$.

8.2 Instantaneous contours of relative total enthalpy in a plane slightly downstream of the rotor passage entrance for the (a) $V I$ and (b) $M P$ cases, normalized by $h_{o 1} \ldots \ldots \ldots \ldots \ldots \ldots \ldots$

8.3 Instantaneous contours of the dimensionless total enthalpy $h_{o} / h_{o 1}$ and relative total enthalpy $h_{o-r e l} / h_{o 1}$ at $70 \%$ span for the $V I$ configuration at times (a) $t / \tau_{r}=0.0$, and (b) $t / \tau_{r}=0.5 \ldots \ldots \ldots \ldots$

8.4 Instantaneous contours of the dimensionless total enthalpy $h_{o} / h_{o 1}$ and relative total enthalpy $h_{o-r e l} / h_{o 1}$ at $70 \%$ span for the $M P$ configuration at (a) $t / \tau_{r}=0.0$ and $(\mathrm{b}) t / \tau_{r}=0.5 \ldots \ldots \ldots \ldots$

8.5 Instantaneous contours of pressure at $70 \%$ span at (a) $t / \tau_{r}=0.0 t / \tau_{r}=0.0$ and (b) $t / \tau_{r}=0.5$, normalized by $P_{o 1}$. Dotted line indicates shock location.

8.6 Instantaneous contours of entropy at $70 \%$ span for the $V I$ configuration at (a) $t / \tau_{r}=0.0$, and (b) $t / \tau_{r}=0.5$, normalized by R. . . . . . . .

8.7 Instantaneous contours of entropy at $70 \%$ span for the $M P$ configuration at (a) $t / \tau_{r}=0.0$, and (b) $t / \tau_{r}=0.5$, normalized by R. . . . . . . . .

8.8 Time-averaged contours of total enthalpy and relative total enthalpy at $70 \%$ span for the (a) VI and (b) $M P$ cases, normalized by $h_{o 1} \ldots \ldots \ldots$

8.9 Rotor section contours of the standard deviation of relative total enthalpy at $70 \%$ span for the (a) $V I$ and (b) $M P$ cases, normalized by $h_{o 1}$. . . . . . .

8.10 Time-averaged contours of entropy in the rotor at $70 \%$ span for the (a) VI and (b) $M P$ cases, normalized by $\mathbf{R} \ldots \ldots \ldots \ldots$

8.11 Contours of entropy standard deviation in rotor at $70 \%$ span for the (a) VI and (b) $M P$ cases, normalized by $\mathbf{R} \ldots \ldots \ldots \ldots$

8.12 Time-averaged static enthalpy contours on the vane surfaces for the (a) VI and (b) $M P$ cases, normalized by $h_{o 1} \ldots \ldots \ldots \ldots$

8.13 Contours of static enthalpy standard deviation on the vane suction side for (a) the $V I$ and (b) $M P$ cases, normalized by $h_{o 1} \ldots \ldots \ldots$

8.14 Time-averaged static enthalpy contours on the blade surfaces for (a) the VI and (b) $M P$ cases, normalized by $h_{o 1} \ldots \ldots \ldots \ldots$ 
8.15 Contours of static enthalpy standard deviation on the blade surfaces for the (a) VI and (b) $M P$ cases, normalized by $h_{o 1} \ldots \ldots \ldots$. . . . . . . 134

8.16 Time-averaged pressure contours on the blade surfaces, normalized by $P_{o 1} \ldots 135$

8.17 Time-averaged relative total enthalpy variation at rotor inlet for the (a) VI and (b) $M P$ cases, normalized by $h_{o 1}$. The view is from downstream of the rotor, with dark lines indicating blade leading edge locations. . . . . . . . .

8.18 Time-averaged relative total enthalpy variation at rotor exit for the (a) VI and (b) $M P$ cases, normalized by $h_{o 1}$. The view is from downstream of the rotor, with dark lines indicating blade trailing edge locations. . . . . . . . .

8.19 Standard deviation of relative total enthalpy at rotor inlet for the (a) $V I$ and (b) $M P$ cases, normalized by $h_{o 1}$. The view is from downstream, with black lines indicating blade leading edge locations. . . . . . . . . . . . . . . 8.20 Standard deviation of relative total enthalpy at rotor exit for the (a) $V I$ and (b) $M P$ cases, normalized by $h_{o 1}$. The view is from downstream of the rotor, with dark lines indicating blade trailing edge locations. . . . . . . . . . . .

8.21 Relative total enthalpy, circumferentially averaged across the inlet of the rotor domain for the (a) $V I$ and (b) $M P$ case, normalized by $h_{o 1}$. The dashed/dotted lines indicate spanwise averages. . . . . . . . . . . .

8.22 Relative total enthalpy, circumferentially averaged over the leading edge surface of the rotor domain for the (a) VI and (b) $M P$ case, normalized by $h_{o 1}$. The dashed/dotted lines indicate spanwise averages. . . . . . . . . . . . . 140

8.23 Designation of rotor blade surface geometry. . . . . . . . . . . . . . . . . 141 
xix

\section{List of Tables}

6.1 Gas properties for air as used in simulations . . . . . . . . . . . 73

7.1 Comparison of mass-weighted total pressure loss coefficients. . . . . . . . . . 96

8.1 Mass-averaged values at the rotor exit, normalized by the corresponding mass-averaged inlet value. . . . . . . . . . . . . . . . 138

8.2 Geometric average of normalized relative total enthalpy for blade surfaces. . 141 


\section{Chapter 1}

\section{Introduction}

Gas turbines are most prevalent in aerospace transportation systems, but are also quite commonly used for propulsion and power generation in marine and land vehicles. They are also common in stationary powerplants and other industrial applications, particularly when a relatively small size and portability are required along with significant power output. Decades of advancements in aerodynamics and materials science have allowed gas turbines to evolve into the engines in use worldwide today, which boast high power-to-weight ratios and high efficiencies. Significant efforts are under way to make further engine design improvements, in order to achieve lower fuel consumption and lower operational costs, as well as longer engine life, higher power output, and reduced size and weight.

\subsection{Motivation}

An important driving force in the continual development of gas turbines is that toward increasingly high combustor temperatures, and therefore higher turbine inlet tem- 
peratures. This translates into higher cycle efficiency and power levels, and has become possible by the use of high-temperature materials and thermal barrier coatings, and by implementing sophisticated cooling strategies. However, inlet temperatures are still limited by the maximum sustainable temperature of the turbine materials. This results in a situation of diminishing practical returns associated with increased cooling flow rate, because the cooling air is extracted from the compressor has a significant effect on the engine's overall performance. In engines with large inlet temperature variations, performance is then further reduced by the practice of designing for higher-than-mean temperatures, as the designer must design for the peak gas temperature when the temperature variation and its effects are not known. If the characteristics and influence of the inlet temperature field are sufficiently understood, the designer can prevent both excessive damage and wear due to higher than predicted temperatures as well as performance losses that arise from an excessively over-cooled turbine section. An effective cooling system in a turbine, especially in the rotor section, is often very complex and difficult to devise and implement (see Fig. $1.1)$.

Turbomachinery designers have traditionally relied upon analytical and empirical flow models based on data which may no longer be applicable to novel designs. The advent of Computational Fluid Dynamics (CFD) has allowed designers to apply much more complex models to turbomachine flow, including models which would previously have required prohibitively lengthy manual calculations. Following a continual growth of available computer resources and the development of more powerful numerical procedures, these flow models have become increasingly sophisticated and effective. Their aim is to predict complex flow 

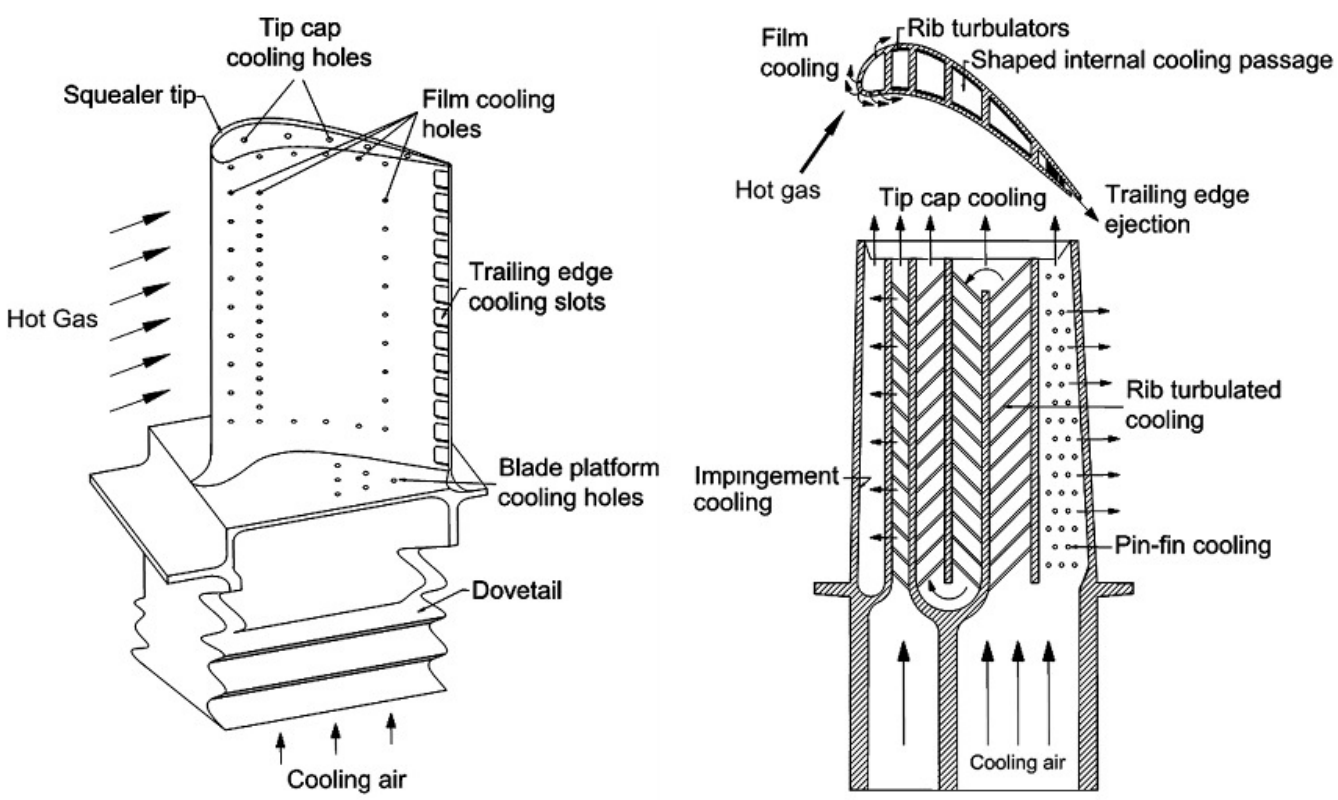

Figure 1.1: Schematic diagram of a modern gas turbine blade with common cooling techniques (Han (2004)).

and heat transfer phenomena with high precision, and thus contribute to improvements in engine design and performance.

CFD analysis necessarily entails the use of many simplifying assumptions. Currently available computing power and models are not yet sufficient to permit one to simulate the operation of an entire engine, and it is necessary to study a single process or a part of the turbomachine separately. While doing so, in principle, it would be necessary to account for the effects of other related processes or interactions with other engine parts. In practice, however, this is only possible in a manner that is simplified, sometimes drastically. Considering a high-pressure turbine stage, the working fluid enters the stage from a duct following the combustors. Gas velocity and temperature in the duct are quite non-uniform, but it is common procedure in turbine simulations to assume that stage inlet conditions correspond 
to uniform mean properties, especially in cases in which determining more realistic flow characteristics would require unavailable experimental data or expensive numerical analysis. Unfortunately, in the case of turbines with large inlet temperature variations, the use of uniform mean properties would likely result in unrealistic results, particularly concerning temperature and heat transfer over different surfaces of the turbine stage. When designing for a component intended to rotate at 16000 RPM and experience 50000 times the acceleration of gravity (Povey et al. (2003)), and with combustor exit temperatures exceeding operating blade temperatures by as much as $1000 \mathrm{~K}$, errors in these predictions can be disastrous.

Gas turbines are typically viewed as having azimuthal periodicity, a concept which generally holds for the fluid flow exiting from a modern annular or can-annular (or tuboannular) combustor. Consequently, the time-averaged inlet temperature field would normally vary along the radial and circumferential directions of the turbine, with the fluid flowing along the axial direction. Experimental data describing the combustor outlet temperature field are significantly easier to obtain than the conditions inside the turbine section, allowing for at the least an estimate of the turbine inlet temperature field if an engine with a comparable combustor is available for testing.

\subsection{Objective and Scope}

The objective of this study is to compare the results of three 3D unsteady simulations of a single-stage high-pressure turbine with inlet boundary conditions corresponding to three different temperature fields of equal mass-averaged mean. The comparison of these 
simulations will serve to evaluate the effects of inlet temperature variation on turbine performance. One simulation has a uniform temperature field at the inlet and will be referred to as the "reference case", as it represents the common type of inlet specification in industrial simulations. In both of the other two cases, the inlet temperature fields contain identical, azimuthally periodical hot spots, but the circumferential positions of the spots are shifted, such that in one case they impact on the stator vanes, whereas in the other they pass between vanes. The commercial CFD code Fluent 6.3.26 has been used for all simulations, and the grid geometry and mesh are identical for all three cases. The simulation geometry and inlet temperature variation were based on data provided by Pratt and Whitney Canada (P\&WC) corresponding to a production turboshaft engine. This compact engine features a reverse-flow combustor (see Fig. 1.2), which produces a characteristic temperature field. The present results will provide information on the effects of employing a more realistic inlet temperature definition than is commonly used, and comparison between the two cases with hot spots will shed light on some of the benefits and drawbacks of each configuration.

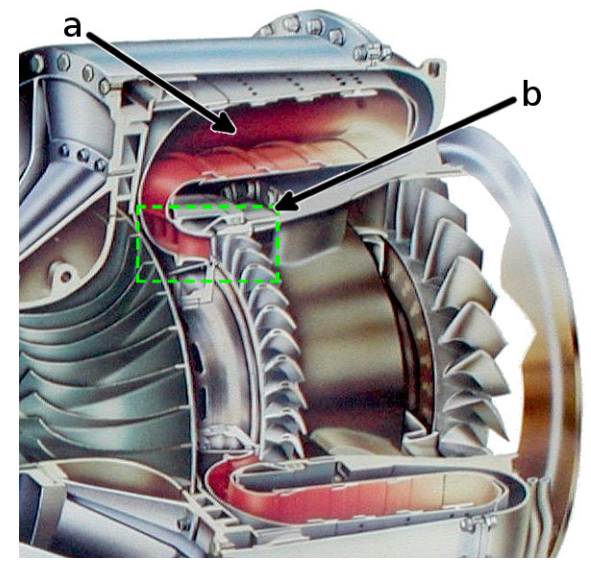

Figure 1.2: Section view of gas turbine portion of a similar turboshaft engine. The reverseflow combustor has been indicated by (a), and the simulated high-pressure stage has been indicated by (b). (Courtesy of the Aircraft Engine Historical Society.) 


\subsection{Outline}

Chapter 2 provides a literature review of some previous work on the subject of hot streaks, primarily focussing on their definition and temperature-related effects. Chapter 3 includes background information on the basics of gas turbine flow, including secondary flows, vortex generation, and associated losses. Chapter 4 summarizes methods of turbulence modelling, numerical analysis, spatial discretization and simulation verification as they relate to this study. Chapter 5 outlines the procedure used for determining appropriate inlet temperature fields for specification as inlet boundary conditions. Chapter 6 summarizes the geometry, its discretization, and the numerical conditions and schemes used for the simulation of the turbine. Chapter 7 presents previously published results of the reference case, using a uniform inlet total temperature, so that they can be used for comparisons with

the results of the present study. Chapter 8 presents the results of the two simulation cases using non-uniform inlet fields and compares these results to each other and to the reference case. Finally, Chapter 9 provides a summary of the hot streak case results, and suggests recommendations for potentially expanding the study. 


\section{Chapter 2}

\section{Literature Review}

This chapter focuses on the available literature on flows through gas turbines with non-uniform inlet temperature. It describes the few available experimental studies and some numerical studies, which complement them. These results would be helpful in determining a sufficiently realistic inlet temperature field without extensive simulation and analysis of the combustor.

The flow of combustion products leaving the combustor is generally quite complex and depends on many factors. Nevertheless, the evolution of temperature variation of this fluid in power systems with similar overall designs is rather insensitive to minor geometric differences. Many published papers are concerned with temperature profiles in conventional 'straight-through' combustion chambers and transition ducts, which are the easiest to analyze and are the most commonly studied. Although the turboshaft engine of interest in the present research is of a different design, using a compact reverse-flow combustor, the general trends observed in conventional systems are still relevant as they can be adapted to 
the present interests following some slight adjustments.

\subsection{Inlet Temperature Distribution}

The common view of the radial temperature profile at the exit of a combustor is that it extends from a relatively low temperature near the walls (due to the injection of cooling air through secondary dilution holes) to its maximum temperature near midspan (Joslyn and Dring (1989)). The radial temperature profile used by Qingjun et al. (2007) in their simulations shows the same trend, with the expected maximum temperature near the blade's midspan falling to relatively low temperatures near the root and tip in a paraboliclike decay.

This trend is supported by the industrial design limits for RTDF (Radial Temperature Distribution Factor) presented by Alkabie (2000), which are typical constraints for the exit plane of a combustion system. Gundy-Burlet and Dorney (1997) simulated hot-streak effects using a hyperbolic-tangent temperature distribution, which also corroborates the general shape of the radial profile. The temperature profile generated in the experiments of Povey et al. (2003) and Povey et al. (2007) follows a similar parabolic-like trend, as does their comparison data taken from an existing engine.

The temperature field at the inlet of a turbine also varies significantly in the circumferential direction, due to the unavoidably discrete elements of combustor geometry, which include fuel injectors and cooling fluid flows. The inlet profiles used in numerical

simulations are usually considerably simplified, as they do not generally incorporate mixing and turbulence factors, but they simply accept that the peak temperatures correspond to 
the location and configuration of combustor and duct geometry (Qingjun et al. (2007)). As the parts of the fluid stream that have a higher temperature, or hot streaks, flow through the transition duct toward the turbine, they are mixed and diffused until they become somewhat less distinct. Both the numerical and experimental combustor exit profiles published by Li et al. (2006) show the existence of distinct hot streaks interacting with the cooler fluid as well as each other. The temperature fields also maintain the radial variation mentioned previously, with the central hot streaks surrounded by cooler regions near the duct boundaries.

The generated inlet temperature fields studied by Povey et al. (2003) and Povey et al. (2007) are quite similar to those found by Li et al. (2006), in that the hot streaks are clearly subject to mixing and diffusion, while maintaining their general position near the centre of the flow field as they get convected downstream. These artificially-produced profiles seem to be marginally more coherent and uniform than those produced by an actual combustor, which is likely due to the assumed conditions that have a lower turbulence and a more uniform geometry than those in the actual case. A temperature profile measured at the exit of a typical military combustor (Povey et al. (2007)) illustrates that, while the hot streaks have diffused together to a much greater extent, the peak temperatures can still be identified and a strong radial gradient persists due to coolant flow. Although it is not clear what type of engine these data represent, they seem to agree with the trend observed in data provided by $\mathrm{P} \& \mathrm{WC}$, specifically that the temperature peaks are skewed toward the duct casing, rather than being centred between the endwalls as in straight-through combustor flow. 


\subsection{Hot Streak Clocking}

When evaluating the effects of inlet hot streaks on an operating turbine section, it is important to note that both the vanes and the rotor blades will be affected. It would be desirable to have an equal number of hot streaks and stator vanes, in order to design the engine in such a way that the vanes will have consistent spatial correlations with these regions of elevated temperature. This arrangement, known as clocking, can be used to direct each hot streak either to the leading edge of the stator vane or to the passage between two vanes, according to the desired effect.

In the case of the hot streaks impinging on the leading edge of the stator vane, the hot streak envelops the vane, thus imparting high temperatures and increasing the heat load on the vane's cooling system. The hot streak is largely diffused as it flows into the rotor section. In the opposite configuration, the hot streak convects predominantly undisturbed past the cooler vane section, then comes into contact with the moving rotor blades and increases their temperature and cooling requirements. The hot streaks are chopped by the rotor and continue as hot spots into the following section of the turbine. These two clocking configurations are widely used for hot streak analysis, as they represent the extremes of interaction between the hot streak and the turbine's surfaces.

In the experiments conducted by Povey et al. (2007), both of these clocking configurations were to be investigated, as well as case with a uniform mean temperature. However, the temperature profiles were positioned in such a way that they were geometrically aligned with respect to the vanes, and the flow field near the leading edge of the vanes was not taken into account. The local flow direction had the effect of shifting the location of the 
peak temperature with respect to the leading edge, specifically toward the suction side of the vane. Thus, the regions of highest and lowest temperatures were in contact with the suction side for the profiles intended to be aligned with and between the vanes, respectively. The temperature of the fluid along the pressure side of the vanes varied much less, remaining at intermediate values in both cases. This oversight highlights the necessity of accurately predicting the displacement effects of the flow directly upstream of the vanes if clocking is to be effectively incorporated into gas turbine design.

\subsection{Buoyancy and Centrifugal Effects}

The presence of a non-uniform temperature field at the turbine inlet influences the flow in the turbine because it introduces density variations, which interact with pressure gradients and rotation effects to give rise to buoyancy and centrifugal forces. These interactions have been investigated by Shang and Epstein (1997), who devised a method for estimating the radial displacement of hot streak fluid by using a set of radial equilibrium equations to evaluate the displacement of a hot streak introduced at the midspan toward the hub.

Shang and Epstein (1997) initially performed a force balance on a fluid element from the peak temperature region of the hot streak as it is transported along the pressure surface. Combined with a number of assumptions based on the nature of the flow and the turbine itself, this analysis yielded a single equation which allows for the estimation of the radial displacement of a hot streak's core fluid. A comparison between this estimation and the core trajectory as computed by a CFD solution showed fairly close agreement, indicating 
that the simple analysis was able to capture the essential physics of this process.

An evaluation of the terms in this equation provided Shang and Epstein (1997) with indications of the nature of buoyancy in the rotor. It was found that the effect scales linearly with hot streak temperature, and that the effect is more pronounced at lower values of the nondimensional flow coefficient. Specifically, buoyancy was found to be inversely proportional to the square of the flow coefficient $\phi^{1}$. It was concluded that the influence of buoyancy, combined with the segregation effect (see Section 3.5.2), tends to move the hot streak toward the hub of the blade. Mathison et al. (2010) also found that the effect of buoyancy tends to promote the spread of higher temperature fluid toward the hub.

\subsection{Published Observations}

Dorney and Gundy-Burlet (1996) found that, for both two- and three-dimensional unsteady simulations, a hot streak impinging on the first-stage stator is convected with the stator's wake, greatly reducing the time-averaged temperatures on the pressure surfaces of the downstream blade rows from those observed with the hot streak passing between stator vanes. Although these early simulations were somewhat simplified, they were among the first to address the problem.

Povey et al. (2007) were able to measure the variation of the heat transfer rates over the surface of the vane (at $50 \%$ span) and the endwalls for each of their experimental configurations. The rate of heat transfer on the pressure side of the vane was insensitive to the inlet profile, which would be expected as this side was not subjected to significantly

\footnotetext{
${ }^{1} \phi=u_{x} / U_{t}$, where $u_{x}$ is the average axial velocity and $U_{t}$ is the rotor blade tip speed.
} 
varying temperatures. The suction side of the vane, however, experienced a substantial increase in the heat transfer rate (over that of the uniform profile) when the hot streak was aligned with the vane leading edge, and a slight decrease in heat transfer when the hot streak was aligned with the mid-passage. This demonstrates that the influence of hot streaks on the heat load of a vane can be quite significant, and underlines the importance of accurately predicting such effects in view of the cost and performance loss associated with internal cooling systems.

In an earlier experiment, Povey et al. (2003) performed a similar test using a hot streak positioned only at the vane mid-passage. These tests indicated an elevated Nusselt number (by 10 to $20 \%$ ) on the pressure surface, while the measurements on the suction surface remained comparable to the results from a study with a uniform temperature profile. As this work used relatively small variations in temperature (only $16 K$ from the average), the temperature variations were not expected to affect significantly the flow of the gases. So it seems that this insensitivity may have been due to a fluid displacement as it enters the vane section, similar to the one in the later study. Thus, the fluid with the highest temperature would then be in contact with the pressure side of the vane, whereas fluid with near-average temperature would be in contact with the suction side.

Another interesting finding of Povey et al. (2007) was that for both cases with non-uniform temperature profiles the Nusselt number (and therefore the heat load) on the duct endwall was significantly lower than the one expected for an inlet flow of uniform temperature with the same mean. This is attributed to the lower temperatures near the duct boundaries that are indicated by the previously mentioned radial temperature profile 
versus a mean uniform profile.

Qingjun et al. (2007) simulated the operation of a single-stage high-pressure turbine coupled with a vaneless counter-rotating low-pressure turbine. When hot streaks impinged on the leading edge of the vanes, they were drawn over the surfaces of the vanes and into the rotor section. There, the hot streaks moved over the surfaces of the blades, with the pressure side experiencing the highest heat loads, predictably increasing with higher peak temperatures. The behaviour of the hot streaks as they migrated past the high-pressure rotor was found to be dominated by the effects of secondary flow and buoyancy, and the combined effects seemed to drive the high-temperature fluid toward the root of the rotor, again increasing with peak temperature ratios.

Although primarily focused on the effects and behaviour of high-swirl vortex cores, the work of Turrell et al. (2004) supports the previously described migration trend, indicating that such a vortex core would be convected along the suction side of a vane and toward the vane's hub, carrying with it the highest-temperature fluid. The spanwise shifting of the core seemed to be due to the pressure gradient within the vane passage. These results, obtained by simulation and also confirmed experimentally, reinforce the importance of understanding the behaviour of the fluid entering the vanes.

Prasad and Hendricks (2000) point out that the secondary flow is driven by the combination of both the rotary total pressure gradient and the centrifugal buoyancy force, which means that the radial migration of hot streaks depends on the relative strengths of these effects. Thus, it is equally possible for the hot streak to migrate toward the hub or the casing, and both trends have been observed by different studies. A method of geometrically 
controlling the secondary flow by altering the vane's twist was also suggested by the same authors; this process effectively changes the exit flow angle along the vane's span, thereby directing the hot streak in the radial direction.

He et al. (2007) found that the interaction between the vane wakes and hot streak heating effects affected the migration and segregation to a larger extent when these two opposing effects were out of phase, as in the case of hot streaks passing between stator vanes. In the case of vane-impinging hot streaks, the segregation was found to be significantly reduced, as the stator wakes ('negative jets') and hot streaks ('positive jets') were in phase and thus largely cancelled each other's effects. They observed this phenomenon clearly in a case with a $1: 1$ ratio of vanes to hot streaks than in another case with a $4: 1$ ratio.

In summary, the results of experimental studies and simulations of flows in gas turbines with temperature variations in both the radial and circumferential directions seem to be in general agreement. These results provide a usable basis on which to define the inlet temperature profile for the present simulations. These distributions also conform with data provided to us by $\mathrm{P} \& \mathrm{WC}$. 


\section{Chapter 3}

\section{Background on Flows in}

\section{High-Pressure Turbines}

This chapter provides background information on some basic concepts of gas turbine flow, associated losses, and the identification of coherent structures. A thorough understanding of these topics is necessary for the analysis and comparative evaluation of the simulation results. The objective of this chapter is to provide a basis on which the contents of subsequent chapters can be communicated effectively.

The flow through even a simple gas turbine is extremely complex due to its threedimensionality, compressibility effects induced by high flow velocity, and the unsteady nature of stator-rotor interactions. The three-dimensionality of the flow field is a result of many interrelated factors, including the following (Lakshminarayana (1996)):

- Radial pressure gradients

- Radial variations in blade shape (blade twist) 
- Compressibility effects

- Blade camber

- Variation in annular area

- Rotor rotation

- Tip and axial gaps

- Inlet velocity and temperature non-uniformities

- Viscous effects in wall regions.

Much of the flow's three-dimensionality is associated with inviscid mechanisms. However, secondary flows are primarily the result of viscous effects. Individual phenomena listed in the previous list interact with each other in many ways to generate even higher complexity. Multiple separation zones, vortices, three-dimensional boundary layers, wakes, and secondary flows are present in gas turbine flows.

\subsection{Shocks}

Shock waves regularly occur in modern high pressure gas turbines (shocks do not form in low pressure turbines). They are typically oblique shocks and produce little energy loss. The shock system of greatest importance is the trailing edge shock system (Denton (1993)). A small triangular recirculation zone is formed directly behind the rounded trailing edge, and the flow from each side of the blade is expanded as it enters this region. Where the suction-side and pressure-side flows meet, a strong shock wave is formed (see Figure 
3.1). The total energy loss due to entropy generation by shocks accounts for approximately $18 \%$ of the total losses for two-dimensional turbine flows (Payne (2001))

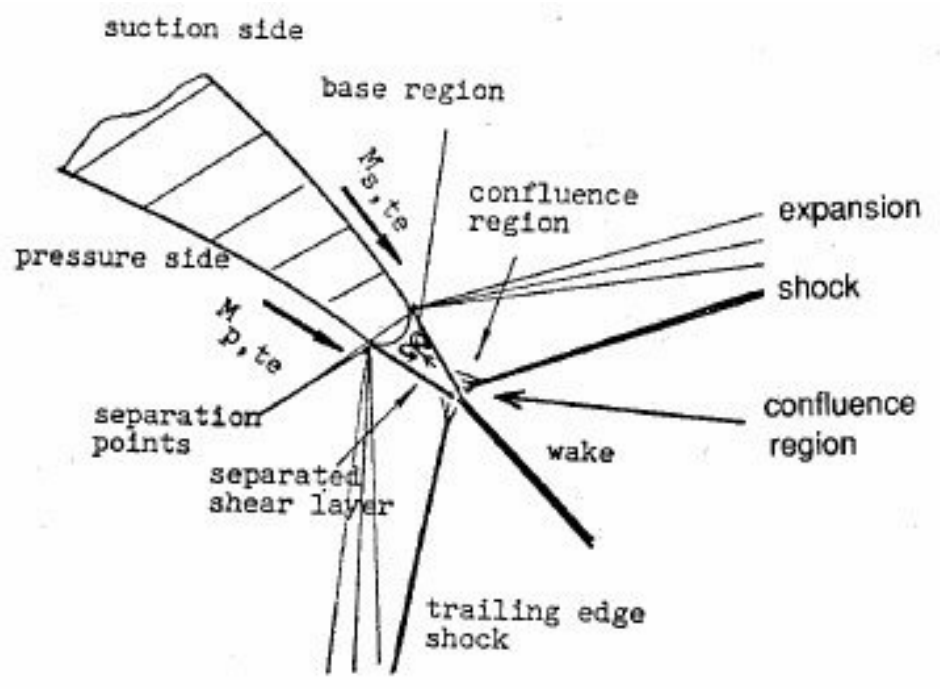

Figure 3.1: Structure of supersonic trailing edge shock system (Denton and Xu (1989)).

Losses occurring in this region have been found to depend on the shape of the airfoil's trailing edge. For instance, elliptical trailing edges produce lower loss than those with square or semi-circular profiles, as the result of differences in dissipation and pressure in the base region (Denton (1993)). In two-dimensional flows with a typical turbulent boundary layer, it has been found that approximately $15 \%$ of the total downstream entropy is generated behind a rotor's thin trailing edge. When the boundary layers are close to separation, this proportion increases to approximately $22 \%$, and it increases further for separated boundary layers and trailing edges of increased thickness (Payne (2001)).

Interactions between shock systems and boundary layers are also important. In the interaction zone, a separation bubble is likely to occur (Denton (1993)). Consequently, 
additional energy losses occur, due to increased dissipation in the separation bubble and downstream of the bubble. Any laminar flow will likely transition to turbulent as it passes this point of separation, and strong shocks may result in complete boundary layer separation (Denton (1993)).

\subsection{Tip Leakage Flow}

Tip leakage flow occurs when a gap exists between the airfoil tip and the casing or hub. By necessity, a gap exists between the rotor blade tip and the outer casing. A gap will also sometimes exist by design between the stator vane tip and a rotating hub. The addition of secondary flows and hub passage vortices complicate the tip leakage flow problem. The leakage flow past the tip is driven by the pressure difference between the suction side and the pressure side of the blade or vane. This pressure difference forces fluid to move from the pressure side of the blade to the suction side of the blade.

The major factors in determining the amount of fluid involved in tip leakage flows are blade loading (because tip leakage depends on the pressure on either side of the blade) and the height of the gap itself. If the tip gap were sufficiently narrow, viscous effects would limit the fluid flow through it. In most cases, however, the tip gap must remain large enough to prevent the rotor tip from contacting the casing when the rotor undergoes expansion due to a high operating temperature. The tip leakage flow rate may therefore be substantial and increases as the gap widens.

In many cases, the leakage flow will roll up and form a vortex on the suction side

of the blade. However, the tip vortex may not form if the turbulence is very high, if the 
mean flow speed is very high, or if a suction-side corner separation zone diffuses the tip leakage flow before it forms a vortex (Lakshminarayana (1996)).

Secondary flows and leakage flows tend to have opposite directions. Leakage flows can be beneficial to gas turbine performance when there is a corner separation region, because the leakage flow pushes the separation region into the main flow field and eliminates it.

Rotor rotation direction significantly impacts the migration of the tip vortex. In a compressor, the rotation assists the tip leakage flow and the tip leakage vortex tends toward the centre of the blade passage. In contrast, the tip leakage flow moves in the same direction as the turbine rotor's rotation, and the tip leakage vortex remains near the suction side of the blade.

In modern, high performance, high-pressure turbines, the tip leakage flow is more important than the secondary flows, with up to $30 \%$ of the losses attributed to the former (Schaub et al. (1993)). The most significant effects of tip leakage flows and tip vortices are the following (Lakshminarayana (1996)):

- They produce a three-dimensional flow field, which can spread away from the tip region, by a distance of approximately 10-30\% of the blade span.

- Tip leakage flow and the associated vortices produce losses, which reduce the device's efficiency.

- The unloading of the blade tip, due to the leakage flow, reduces the pressure change through the rotor and reduces the angle through which the flow turns. 
- They cause unsteadiness, which can lead to higher stresses on downstream blades, vibration, flutter, and noise production.

- They introduce complications to the heat transfer process.

\subsection{Secondary Flows}

Secondary flows are the result of viscous effects in the presence of velocity, pressure and temperature gradients introduced by boundary layers on the walls and other upstream surfaces. Lakshminarayana (1996) gives a simplified description of secondary flow generation by viscous effects in a boundary layer for a steady incompressible $2 \mathrm{D}$ cascade. Figure 3.2 shows the simplified blade channel used in this explanation. Line $A A A$ is a streamline in the inviscid region and line $B B B$ is another streamline, directly below $A A A$, in the boundary layer region. For simplicity, velocity gradients and viscous effects in the $n$ (pitchwise) direction will be neglected. If the flow is incompressible and steady (no rotation), the pressure gradient in the normal direction $n$ at a point along streamline $A A A$ will be proportional to the centripetal acceleration, as

$$
\left(\frac{\partial P}{\partial n}\right)_{A}=\rho \frac{u_{A}^{2}}{R_{A}}
$$

where $R$ is the local radius of curvature. If we assume that the pressure is constant across the boundary layer, then the normal pressure gradient would have the same value for streamlines $A A A$ and $B B B$, so that

$$
\left(\frac{\partial P}{\partial n}\right)_{A}=\left(\frac{\partial P}{\partial n}\right)_{B}=\rho \frac{u_{A}^{2}}{R_{A}}>\rho \frac{u_{B}^{2}}{R_{B}}
$$




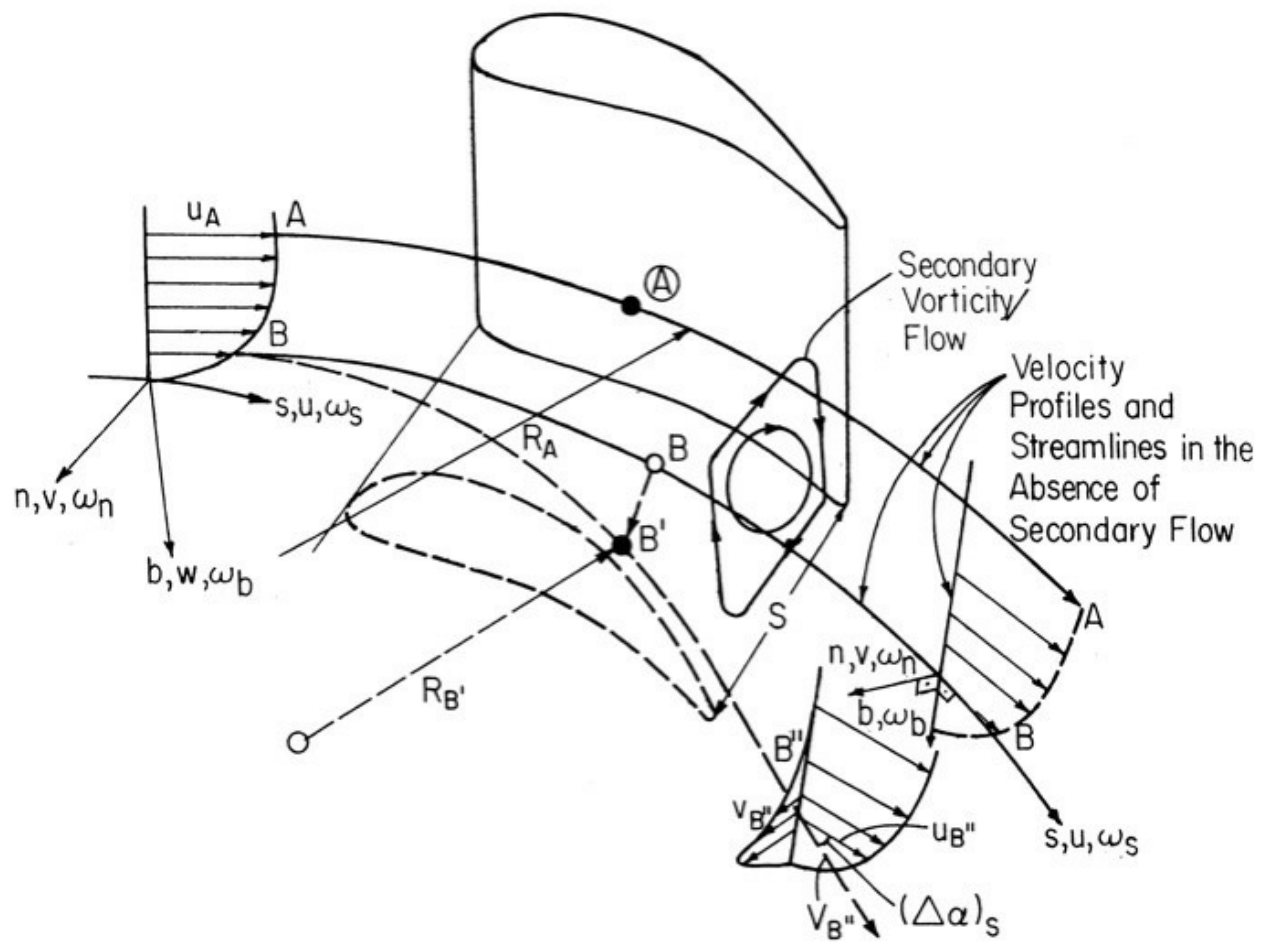

Figure 3.2: Schematic illustration of the development of secondary flow in a rotor blade passage (Lakshminarayana (1996)).

However, because $u_{B}<u_{A}$ and $R_{B}=R_{A}$, the normal pressure gradient along streamline $B B B$ would not be proportional to the centripetal force that a fluid particle would experience moving with a velocity $u_{B}$ at a radius of $R_{B}$. As a result, the streamline $B B B$ would be deflected to $B B^{\prime} B^{\prime \prime}$, to permit the normal pressure gradient force to balance the centripetal force. This results in a cross flow $v$, which is known as secondary flow. Moore and Adhye (1985) defined secondary flow as unplanned three-dimensional flow effects in a turbine which lead to unexplained (secondary) losses.

An important effect of the secondary flow is that of vorticity generation. In in- 
compressible flow, the continuity equation is

$$
\frac{\partial u}{\partial s}+\frac{\partial v}{\partial n}+\frac{\partial w}{\partial b}=0
$$

In steady flow, along the mean line of a curved duct (neglecting the streamwise pressure gradient in the $s$ direction) $\partial u / \partial s=0$ (Lakshminarayana (1996)), therefore

$$
\begin{aligned}
\frac{\partial v}{\partial n} & =-\frac{\partial w}{\partial b} \\
\omega_{s} & =-\frac{\partial v}{\partial b}+\frac{\partial w}{\partial n}
\end{aligned}
$$

This relation shows that viscous effects in the boundary layer give rise to additional velocity components $(v$ and $w)$, which lead to secondary flow and streamwise vorticity $\omega_{s}$ (Equation 3.5) in a gas turbine blade passage.

It is clear that a secondary flow field will develop when a flow with a velocity gradient (e.g., a boundary layer or a vortex) moves through a curved path, such as a blade passage. Secondary flows can also occur when viscous flows interact with the leading edge of a blade or vane or when there exists a radial temperature gradient at the inlet. This can be demonstrated by using a generalized analysis of the vorticity equations (Lakshminarayana (1996)), although the detailed analysis was omitted here. The generalized equations for vorticity give the following expression modeling compressible flow, with some simplifying assumptions, for the vorticity in the streamwise direction $s$ :

$$
\frac{\partial}{\partial s}\left(\frac{\omega_{s}}{\rho u}\right)=\frac{2 \omega_{n}}{\rho U R}+\frac{1}{\rho^{2} R} \frac{\partial \rho}{\partial b}=\frac{2 \omega_{n}}{\rho U R}-\frac{1}{\rho R T} \frac{\partial T}{\partial b}
$$

where $\rho$ is density, $\omega_{n}$ is the vorticity in the $n$ direction and $T$ is temperature. The first term on the right hand side shows the contribution of the velocity gradient in the spanwise direction $b$ to the streamwise vorticity, as discussed previously. The second term shows 
the effect of a spanwise temperature gradient on the streamwise vorticity. This was not observed in Equation 3.5. The previous equation can be simplified to

$$
\frac{\partial}{\partial s}\left(\frac{\omega_{s}}{\rho U}\right)=\frac{2}{\rho \rho_{\infty} U^{2} R} \frac{\partial P_{0}}{\partial b}
$$

Equations 3.6 and 3.7 show that it is possible to manipulate both the spanwise temperature gradient and the inlet spanwise velocity gradient, such that the two terms on the right hand side of equation 3.6 cancel each other out. This leads to a reduced spanwise stagnation pressure gradient, which reduces the streamwise vorticity and secondary flow, as indicated by equation 3.7 .

In addition to the previous effects, secondary flows can be also generated by rotation of the rotor and by compressibility effects. However, in axial gas turbines the rotation of the rotor has negligible effect on secondary flows. In contrast, compressibility effects change the inlet vorticity $\omega_{n}$ and the temperature gradient $\partial T / \partial b$, which in turn affect the secondary flow field significantly.

In general, secondary flows have several effects on gas turbine flows, including the following:

- Generation of cross flow, resulting in a three-dimensional flow field.

- An effect on the flow turning, which affects the pressure change through gas turbines.

- Secondary flow losses, which result in approximately a $2-4 \%$ reduction in efficiency.

- The generation of a vortex by the cross flow, which may cause flow separation in the corner region where the end wall and the blade suction surface meet. 
- An effect on the flow conditions through downstream rotors and stators, which results in unsteadiness and possibly flutter, vibration and noise.

- Distortion of the temperature field.

\subsection{Passage Vortices}

The hub passage vortex is generated from the pressure-side leg of the horseshoe vortex that develops due to the approach of the incoming boundary layer upon the junction between the blade or vane leading edge and the endwall. These vortices are shown in Figure 3.3, which illustrates the secondary flow model of Takeishi et al. (1990). There are many secondary flow models, but they do not all agree on the existence of the smaller corner vortices (Goldstein and Spores (1988)) and the path that the suction leg of the horseshoe vortex follows. However, all investigators agree that the passage vortex is the most dominant and strongest feature of the flow in this region, as the absence of a gap precludes the formation of a tip vortex. Moreover, the pressure-side leg of the horseshoe vortex is much stronger than its suction-side leg due to the amplification of the former by the crossflow component near the endwall, whereas secondary crossflow weakens the latter. As seen in Figure 3.3, the passage vortex travels across the blade passage towards the suction side of the adjacent blade. The suction-side leg of the horseshoe vortex (identified by the term 'counter vortex' in Figure 3.3) is thought to either orbit around the passage vortex (Sharma and Butler (1987)) or travel outwards in the spanwise direction toward the above casing (Goldstein and Spores (1988); Takeishi et al. (1990)) or pass below the passage vortex (Langston (1980)). 


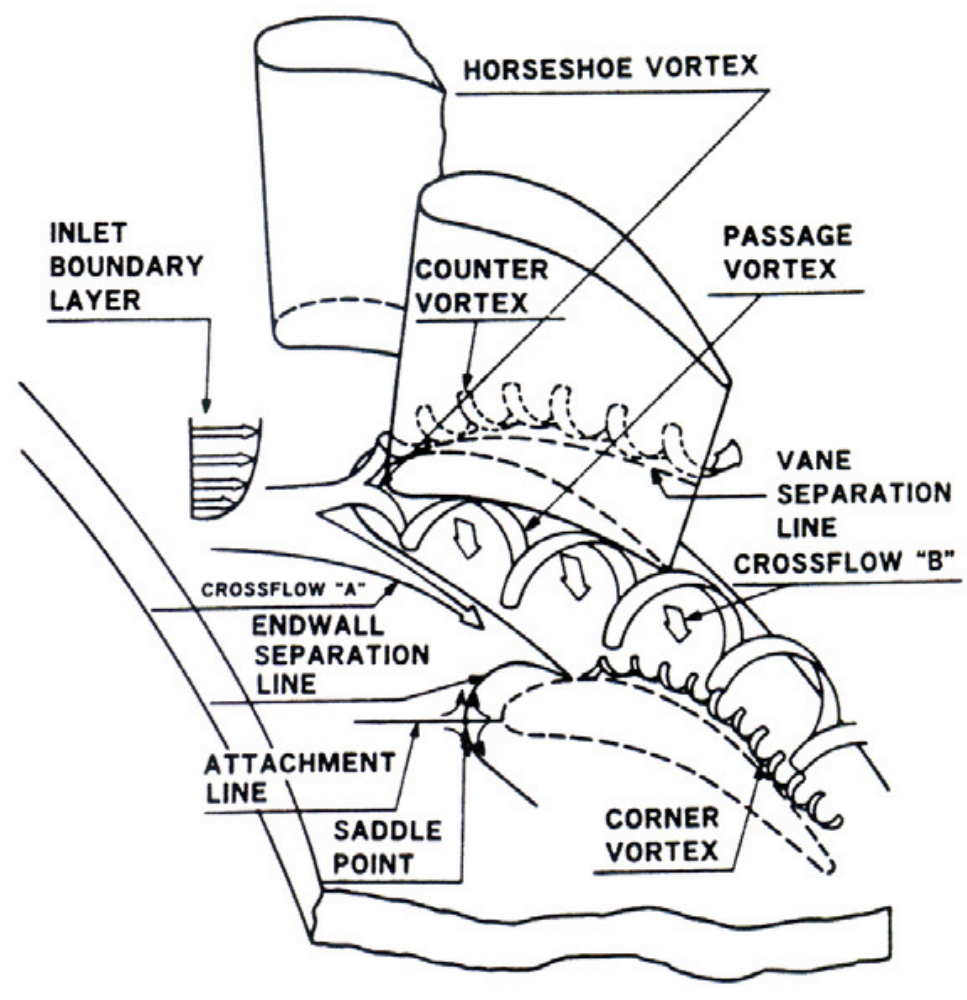

Figure 3.3: Passage vortex model of Takeishi et al. (1990).

The horseshoe vortex develops by the interaction of the incoming boundary layer and the spanwise increase in pressure along the leading edge of the vane or blade with increased radius. This spanwise pressure gradient drives the fluid at the leading edge toward the endwall and into the boundary layer, forming a vortex, the ends of which are convected downstream to form a shape resembling a horseshoe. The strength and structure of the horseshoe vortex have been found to depend on the leading edge radius of the blade or vane. If the leading edge radius is greater than the boundary layer thickness, the horseshoe vortex is formed as a large single vortex, as shown in Figure 3.4a. If the leading edge has a smaller radius than the boundary layer thickness, the horseshoe vortex consists of 
a combination of several smaller vortical structures, as shown in Figure 3.4b (Xun et al. (2005)). As a single large vortex is stronger than the combination of smaller vortices, a large leading edge radius will lead to a single strong horseshoe vortex and higher losses, which may be reduced by decreasing the leading edge radius. If the radius is sufficiently reduced, the horseshoe vortex may be close enough to the endwall surface to be diffusively eliminated by viscous stress in the boundary layer (Bradshaw (1987)).

On the opposite side, Roulund et al. (2005) found that a cylindrical pile may not generate a horseshoe vortex if the boundary layer thickness is less than $1 \%$ of the pile's diameter. Therefore, accelerating the flow through a subsonic nozzle may reduce the horseshoe vortex by thinning the boundary layer. This is not usually the case, as the blade leading edge radius is commonly designed to be sufficiently large in order to reduce incidence sensitivity, and to improve heat transfer and thus blade cooling.

It is generally agreed upon that the passage vortex is a dominant structure in turbomachine flows. Niestroj and Came (1998) found that the passage vortex strength is increased in turbine blade rows when rotation is applied. The increased losses in a rotating blade row over those in a stationary blade row emphasize the significant contribution of the passage vortex to the losses of turbomachines.

\subsection{Effects of Temperature Nonuniformity on Flow Distrib- ution}

In this section, we discuss briefly some effects which result in significant inlet temperature redistribution in the turbine rotor and possibly introduce localized overheating 


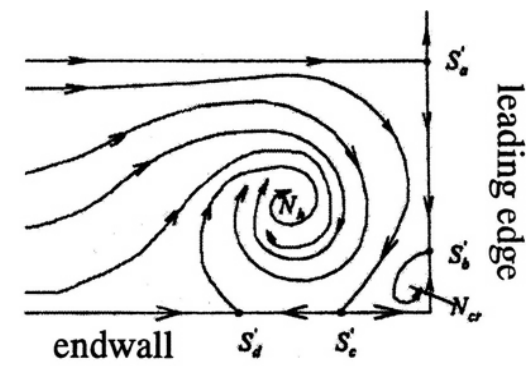

a) Leading edge radius larger than boundary thickness

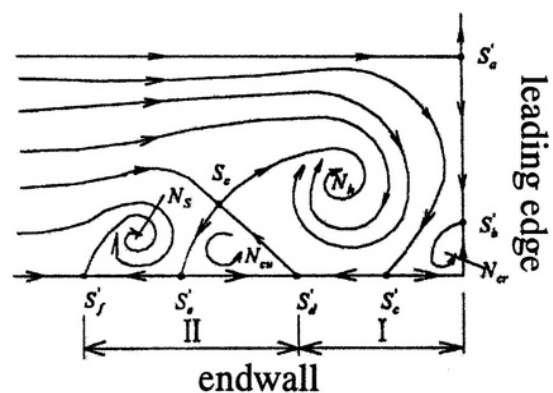

b) Leading edge radius smaller than boundary thickness

Figure 3.4: Horseshoe vortex topology (Xun et al. (2005)).

where the hotter gas makes contact with the endwall and airfoil surfaces. In addition, when circumferential variations in inlet temperature are present, they contribute to the unsteady loading of the rotor and possibly accelerate structural fatigue.

\subsubsection{Substitution Principle}

Munk and Prim (1947) theoretically demonstrated that the introduction of total temperature non-uniformity (e.g., by hot streaks) at the inlet alone will not alter the streamline pattern in a stationary blade row, provided that the total pressure is uniform over the inlet boundary. This leads to the expectation that temperature redistribution will only occur due to secondary flow in the stator if both inlet total temperature and total pressure nonuniformities are present. Further secondary flow analyses, however, indicate that temperature nonuniformities at the inlet can lead to secondary flow in the rotor section (Hawthorne (1951)). The nonuniform inlet temperature profile can induce a normal component of relative vorticity at the rotor inlet, which is subsequently converted to streamwise 
vorticity inside the rotor section, resulting in secondary flow.

\subsubsection{Positive and Negative Jets}

These two opposing movements can suppress each other, depending on the hot streak's clocking position, and phase differences between the two lead to variation in the thermal effects observed on the rotor blades. These cumulative effects have been described by He et al. (2007) for different ratios between the number of stator vanes and the number of hot streaks.

\section{Segregation Effect}

Butler et al. (1989) demonstrated experimentally that, in a blade row, an inlet total temperature nonuniformity can lead to a segregation of hot and cold gases across the airfoil, caused by differences in the local approaching flow angle between the hot and cold fluid. The result is that fluid of higher temperature migrates toward the pressure side of the blade, and fluid of lower temperature is convected toward the suction side. This cross-passage movement, sometimes called preferential heating (He et al. (2007)), can be regarded as a positive jet, and has been computationally confirmed by Dorney et al. (1992). In general, hot streaks near the midpassage of the stator vanes have been found to produce the strongest effects. Although total temperature segregation has not been proved to have a direct impact on aerodynamic performance (Adamczyk (2000)), the differential heating of blade pressure and suction sides is significant. 


\section{Wakes}

In a turbine stage, a wake shed from the upstream stationary row contains highloss fluid, which will be convected from the pressure to the suction surface of the rotating blade. This phenomenon is often called a negative jet (He et al. (2007)).

The losses in the wake region have been found to depend on the shape of the trailing edge (Payne (2001)). Elliptical trailing edges reduce the loss with respect to square or circular trailing edges. It has been found, for two-dimensional flows, that approximately 15 to $22 \%$ of the total loss is due to the wake behind a rotor blade with a trailing edge of zero thickness (Payne (2001)). The addition of a thick trailing edge can increase this loss by approximately $12 \%$ up to a total wake loss of $27 \%$ to $34 \%$ (Payne (2001)).

\subsection{Qualification of Losses, Efficiency and Flow Coefficients}

In industrial practice, the estimation of losses is usually accomplished through the use of empirical correlations. However, in the interest of accuracy, the present study endeavours to predict the losses directly from the numerical solutions and without the use of correlations. Typically, the losses are represented by the total pressure loss coefficient (Schobeiri (2005))

$$
Y_{p}=\frac{P_{01}-P_{02}}{P_{02}-P_{2}}
$$

where the subscripts 01,02 , and 2 indicate the stagnation property at state 1 , the stagnation property at state 2 , and the static property at state 2 , respectively. This parameter is widely used because it is easily determined from experimental data. However, for a turbine blade, 
a more appropriate loss coefficient is (Denton (1993))

$$
\zeta_{h}=\frac{h_{2}-h_{2 s}}{h_{02}-h_{2}}
$$

where $h$ is specific enthalpy and the subscript $2 s$ indicates the static property at state 2 after an isentropic process. Although the use of the loss coefficients defined by equations 3.8 and 3.9 is common, it may not be appropriate in the detailed analysis of rotating blades, because the rotation can produce changes in the relative stagnation pressure and enthalpy without incurring any corresponding losses (Denton (1993)).

Denton (1993) suggests that the estimation of both losses and efficiency be accomplished through entropy analysis, because the entropy value is independent of the frame of reference. However, in order to determine the efficiency, the entropy $s_{1}$ at a reference state is required. In experimental studies, this would require the measurement of many variables, which may not be easy to achieve. Fortunately, this is not a problem for a numerical study, as all necessary local properties for any location in the domain are part of the solution. The specific entropy change can be determined as (Van Wylen and Sonntag (1978))

$$
\frac{s_{2}-s_{1}}{\mathbf{R}}=\left(\frac{\gamma}{\gamma-1}\right) \ln \left(\frac{T_{02}}{T_{01}}\right)-\ln \left(\frac{P_{02}}{P_{01}}\right)
$$

where $\mathbf{R}$ and $\gamma$ are the gas constant and the ratio of specific heats, respectively, of the working fluid. For a turbine stage, the working fluid is the mixture of the products of combustion (see Section 6.3 for the specified properties). The total-to-total (isentropic) efficiency $\eta_{t t}$ is defined as the ratio of the actual turbine work output and the isentropic 
turbine work output

$$
\begin{aligned}
\eta_{t t} & =\frac{h_{01}-h_{02}}{h_{01}-h_{02 s}}, \text { or } \\
\eta_{t t} & =\frac{T_{01}-T_{02}}{T_{01}\left[1-\left(\frac{P_{02}}{P_{01}}\right)^{\frac{\gamma-1}{\gamma}}\right]}, \text { if } \mathbf{C}_{\mathbf{p}} \text { is constant }
\end{aligned}
$$

where the subscript $02 s$ indicates the stagnation property at state 2 after an isentropic process. This is accurate if there is no heat transfer with the surroundings. In many cases, kinetic energy can be neglected and the isentropic efficiency can be simplified to

$$
\eta_{t t} \simeq \frac{h_{1}-h_{2}}{h_{1}-h_{2 s}}
$$

The isentropic work output can be written in terms of entropy and temperature instead of enthalpy change after an isentropic process, if the temperature is assumed to be constant between states 2 and $2 s$ (Denton (1993))

$$
\eta_{t t} \simeq \frac{h_{1}-h_{2}}{h_{1}-h_{2}+T_{2}\left(s_{2}-s_{1}\right)}
$$

Denton (1993) states that this assumption is not likely to result in large errors. The totalto-static efficiency $\eta_{t s}$ is defined as the actual turbine work output divided by the isentropic turbine work output and the kinetic energy of the exit flow of an ideal turbine

$$
\begin{aligned}
\eta_{t s} & =\frac{h_{01}-h_{02}}{\left(h_{01}-h_{02 s}\right)+\frac{1}{2} U_{2}^{2}}=\frac{h_{01}-h_{02}}{h_{01}-h_{2 s}} \\
\eta_{t s} & =\frac{T_{01}-T_{02}}{T_{01}\left[1-\left(\frac{P_{2}}{P_{01}}\right)^{\frac{\gamma-1}{\gamma}}\right]}, \text { if } \mathbf{C}_{\mathbf{p}} \text { is constant }
\end{aligned}
$$

where $U$ is the flow velocity magnitude. A comparison of the two efficiency definitions shows that the total-to-static efficiency treats the kinetic energy contained in the exit as a loss, whereas the total-to-total efficiency does not. As a result, the total-to-total efficiency 
equation is a more accurate representation of the efficiency of multistage gas turbines and the total-to-static efficiency is a better representation of the efficiency of single stage turbines or of the last stage of a multi-stage gas turbine (Lakshminarayana (1996)).

The entropy loss coefficient, defined by Denton (1993), is

$$
\begin{aligned}
\zeta_{s} & =\frac{T_{2}\left(s_{2}-s_{1}\right)}{h_{02}-h_{2}} \\
\zeta_{s} & =\frac{s_{2}-s_{1}}{\mathbf{C}_{\mathbf{p}}\left(\frac{T_{02}}{T_{2}}-1\right)} \text { if } \mathbf{C}_{\mathbf{p}} \text { is constant }
\end{aligned}
$$

The generation of entropy in fluid processes is generally inversely proportional to the local temperature, as shown by Denton (1993)

$$
T \Delta s=\zeta_{s} \frac{1}{2} U^{2}
$$

In addition, because the loss coefficient is essentially independent of temperature, flows with constant velocity and a constant entropy loss coefficient produce less entropy at higher temperatures than would be the case if the same process was to occur at a lower temperature. It is therefore beneficial, in terms of efficiency, to operate a gas turbine at higher temperatures.

It is easier to identify the sources of energy losses by dividing them into groups, each of which contains a major loss feature. A traditional method, used by Denton (1993), divides the losses into three groups:

- Profile losses,

- Secondary flow and endwall losses, and

- Leakage losses. 


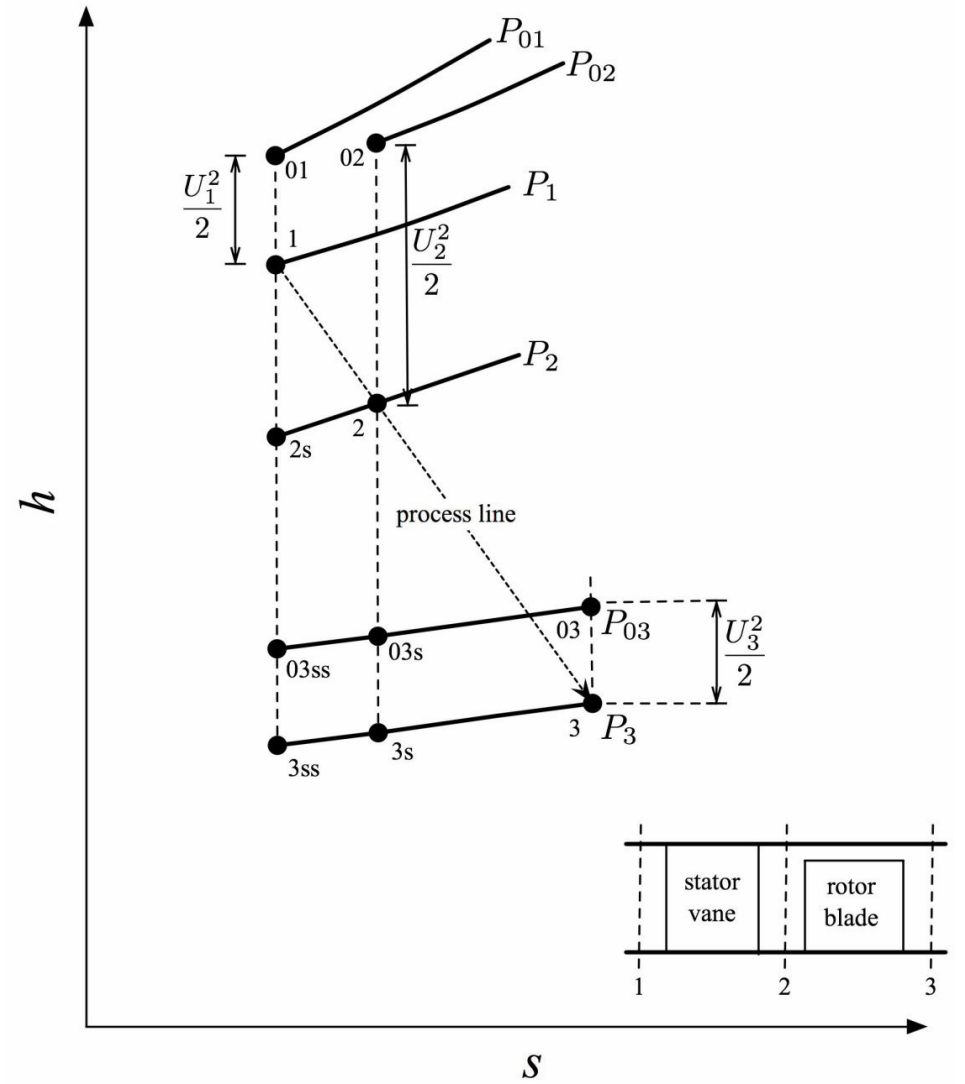

Figure 3.5: Enthalpy-entropy diagram for the expansion process in a single-stage turbine (based on Lakshminarayana (1996)).

Profile losses include losses that are due to blade boundary layers and mixing near the trailing edge. Secondary flow losses occur throughout the blade passage and endwall losses occur near the hub and casing endwalls. Secondary flow and endwall losses are difficult to separate from the profile losses and leakage losses because they are generated by many different mechanisms. Therefore, the secondary flow and endwall losses category is often used to account for losses not included in the profile and leakage losses. Leakage losses denote those due to flow passing through the tip gap region bounded by the blade (or vane) tips and endwalls. Typically, the magnitudes of the three types of losses are approximately 
equal, so that each type accounts for approximately 1/3 of the total losses (Denton (1993)).

Other methods of loss breakdown have been developed as well. Payne (2001) separated the losses into five categories, as follows:

- Profile losses,

- Wake/mixing losses,

- Shock losses,

- Secondary flow and endwall losses, and

- Leakage losses.

This method allows for a more detailed categorization of the losses in a gas turbine, however, it is very difficult to determine the magnitude of each loss type, because of the interactions between them. For example, the interaction between shocks and boundary layers generates additional losses, which cannot be classified in any of the previously mentioned groups. However, many loss correlations attempt to predict the losses associated with the above groups.

Though numerous correlations and theories exist for predicting the losses associated with the profile, wake, and shocks, the three-dimensionality of the flow and the inter-connectivity between various losses make it difficult to determine the contribution of each mechanism to the overall loss. Denton (1993) accomplished this by considering numerical results at a plane downstream of the rotor of interest. This plane was then split into three zones:

- The primary flow field, 
- Tip leakage flow, and

- The remaining secondary flow field.

The primary flow field consists of low entropy fluid. This region is defined as all fluid with a lower specific entropy than a set threshold. The threshold value separates the low entropy fluid (primary flow field) from the high entropy fluid (tip leakage flow and remaining secondary flow). Once the three regions have been determined, the mass-averaged entropy can be computed (Payne (2001)) as

$$
\frac{\widehat{s}}{\mathbf{R}}=\frac{\int\left(\frac{s}{\mathbf{R}}\right) \rho U d A}{\int \rho U d A}
$$

This value can then be used to determine the loss coefficients and the stage efficiency. This method does not separate all major loss mechanisms, but was found to be the best available method which distributes the losses due to the three major mechanisms from numerical results, without the use of loss correlations. 


\section{Chapter 4}

\section{Numerical Background}

This chapter introduces some relevant methods of turbulence modelling, numerical analysis, spatial discretization and simulation verification. These topics are essential in understanding the simulation procedures and analysis results.

\subsection{Governing Equations}

For an accurate simulation of flows in gas turbines, one must account for the unsteadiness and the turbulence, whose effects dominate the flow. Among the available Computational Fluid Dynamics (CFD) methods, direct numerical simulations (DNS) and large eddy simulations (LES) are considered to be the most accurate, but they are not suitable for practical simulations of flows in entire engines or even in turbine stages, because they require a very high mesh density, which cannot generally be handled by current computers. The number of mesh elements (e.g., finite volume nodes) $N_{D N S}$ required by DNS is related to the Reynolds number Re as $N_{D N S} \sim \operatorname{Re}^{2.25}$ (Blazek (2005)). A simulated high-speed 
gas turbine flow with a Reynolds number of the order $10^{6}$ would then require a grid with a number of nodes of the order $10^{13.5}$. The approximate LES node count requirement is given by $N_{L E S, o} \sim \operatorname{Re}^{0.4}$ in the outer regions and $N_{L E S, i} \sim \mathrm{Re}^{1.8}$ in the viscous sublayer (Blazek (2005)). For the same gas turbine and Reynolds number discussed previously, this yields a mesh with $10^{13.2}$ nodes. Both of these node counts greatly exceed the available computing power, necessitating a less computationally intensive approach. Such an available approach is the solution of Unsteady Reynolds Averaged Navier Stokes (URANS) equations combined with the use of turbulence models.

\subsubsection{Unsteady Reynolds Averaged Navier-Stokes Equations}

In the present analysis, the simulations produce as output a part $u_{i}$ of the timedependent local velocity, to be called the resolved velocity, as well as resolved pressure $P$, temperature $T$, density $\rho$ etc.. Using the conventional Reynolds averaging method, a resolved property is considered as composed of a mean component, averaged over an ensemble of realizations (denoted by angle brackets) and a fluctuating component (denoted by a prime), as in the following example:

$$
u_{i}=\left\langle u_{i}\right\rangle+u_{i}^{\prime}
$$

Ensemble averages in the present flows, which are periodic on the average, are obtained as phase averages by averaging all values of the corresponding property at the same relative time instant during each cycle.

In addition to the resolved component, each actual instantaneous local flow prop- 
erty contains an unresolved component (denoted by a double prime), as for example

$$
u_{i, a c t u a l}=u_{i}+u_{i}^{\prime \prime}=\left\langle u_{i}\right\rangle+u_{i}^{\prime}+u_{i}^{\prime \prime}
$$

The unresolved components are considered to represent relatively small-scale turbulent fluctuations, whose scales are smaller than the computational mesh size. The unresolved components are taken into account in a statistical sense only by the use of turbulence models. In the following, statistical moments of unresolved properties will be denoted by braces, e.g., $\left\{u_{i}^{\prime \prime 2}\right\}$. The use of a specific turbulence model would provide estimates of the timedependent local statistical moments of unresolved properties, as for example an instantaneous local turbulent kinetic energy per unit mass $k$

$$
k=\frac{1}{2}\left[\left\{u_{1}^{\prime \prime 2}\right\}+\left\{u_{2}^{\prime \prime 2}\right\}+\left\{u_{3}^{\prime \prime 2}\right\}\right]
$$

The total ensemble-averaged kinetic energy of all velocity fluctuations at any given phase in the cycle would be

$$
\left\langle k_{t o t}\right\rangle=\langle k\rangle+\frac{1}{2}\left[\left\langle u_{1}^{\prime 2}\right\rangle+\left\langle u_{2}^{\prime 2}\right\rangle+\left\langle u_{3}^{\prime 2}\right\rangle\right]
$$

The momentum equation for the resolved properties, expressed in index notation, is (Ansys (2006a))

$$
\frac{\partial}{\partial t}\left(\rho u_{i}\right)+\frac{\partial}{\partial x_{j}}\left(\rho u_{i} u_{j}\right)=-\frac{\partial P}{\partial x_{i}}+\frac{\partial}{\partial x_{j}}\left[\mu\left(\frac{\partial u_{i}}{\partial x_{j}}+\frac{\partial u_{j}}{\partial x_{i}}-\frac{2}{3} \delta_{i j} \frac{\partial u_{k}}{\partial x_{k}}\right)\right]+\frac{\partial}{\partial x_{j}}\left(-\rho\left\{u_{i}^{\prime \prime} u_{j}^{\prime \prime}\right\}\right)
$$

The Reynolds stress $-\rho\left\{u_{i}^{\prime \prime} u_{j}^{\prime \prime}\right\}$ accounts for the unresolved fluctuations and $\delta_{i j}$ is the Kronecker delta, equal to 1 when $i=j$ and equal to 0 when $i \neq j$.

The conservation of energy equation is (Ansys (2006a)) 


$$
\frac{\partial}{\partial t}(\rho e)+\frac{\partial}{\partial x_{j}}\left(u_{j}[\rho e+P]\right)=\frac{\partial}{\partial x_{j}}\left[\kappa_{e f f} \frac{\partial T}{\partial x_{j}}\right]+\frac{\partial}{\partial x_{j}}\left[u_{i} \mu_{e f f}\left(\frac{\partial u_{i}}{\partial x_{j}}+\frac{\partial u_{j}}{\partial x_{i}}-\frac{2}{3} \delta_{i j} \frac{\partial u_{k}}{\partial x_{k}}\right)\right]
$$

The specific internal energy $e$, the effective thermal conductivity $\kappa_{e f f}$, and the effective dynamic viscosity $\mu_{\text {eff }}$ are defined as

$$
\begin{gathered}
e=h-\frac{P}{\rho}+\frac{U^{2}}{2} \\
\kappa_{e f f}=\kappa+\kappa_{t} \\
\mu_{e f f}=\mu+\mu_{t}
\end{gathered}
$$

where $h, \kappa, \kappa_{t}, \mu$ and $\mu_{t}$ are, respectively, the specific enthalpy, the fluid thermal conductivity, the turbulent thermal conductivity, the fluid dynamic viscosity, and the turbulent dynamic viscosity; the instantaneous local velocity magnitude is defined as

$$
U=\left(u_{1}^{2}+u_{2}^{2}+u_{3}^{2}\right)^{\frac{1}{2}}
$$

The first term on the left hand side of Equation 4.6 represents the temporal rate of change of energy, whereas the second term represents the convection of energy by the flow. The two terms on the right hand side of Equation 4.6 represent molecular diffusion of heat and viscous heating, respectively. The viscous heating term models the thermal energy produced by the viscous shear and is always included when the coupled solver is used in the CFD code used presently. Viscous heating becomes important in flows with a relatively high Mach number (Clark et al. (2000)).

The continuity equation for a compressible flow is (Ansys (2006a)) 


$$
\frac{\partial \rho}{\partial t}+\frac{\partial}{\partial x_{i}}\left(\rho u_{i}\right)=0
$$

\subsubsection{Favre Averaged Navier-Stokes Equations}

Considering that the flows of interest are compressible, it is necessary to implement a density-based solver. This solver, in conjunction with the requirement of unsteady turbulence modelling, requires the use of the Unsteady Favre Averaged Navier Stokes (UFANS) equations. The UFANS equations are derived from the Navier Stokes equations by using Favre averaging, namely by using the density-weighted mean velocity (Hinze (1975)), defined as

$$
\begin{aligned}
\widetilde{u}_{i} & =\frac{\left\langle\rho u_{i}\right\rangle}{\langle\rho\rangle} \\
\rho & =\langle\rho\rangle+\rho^{\prime}
\end{aligned}
$$

In solving the UFANS equations, Reynolds averaging is used for density and pressure, while Favre averaging is used for velocity, internal energy, enthalpy, and temperature.

After Favre averaging, the conservation of momentum becomes

$$
\frac{\partial}{\partial t}\left(\rho \widetilde{u_{i}}\right)+\frac{\partial}{\partial x_{j}}\left(\rho \widetilde{u_{i}} \widetilde{u_{j}}\right)=-\frac{\partial P}{\partial x_{i}}+\frac{\partial}{\partial x_{j}}\left[\mu\left(\frac{\partial \widetilde{u_{i}}}{\partial x_{j}}+\frac{\partial \widetilde{u_{j}}}{\partial x_{i}}-\frac{2}{3} \delta_{i j} \frac{\partial \widetilde{u_{k}}}{\partial x_{k}}\right)\right]+\frac{\partial}{\partial x_{j}}\left(-\rho\left\{u_{i}^{\prime \prime} u_{j}^{\prime \prime}\right\}\right)
$$




\subsubsection{Choice of CFD Code}

The necessity for using a density-based solver partially influenced the choice of software package for use in these simulations. In deciding between the two available CFD codes, FLUENT and CFX, it was determined that CFX did not incorporate a density-based coupled solver. It also lacked a local mesh-refinement feature, as well as a pressure-based segregated solver, though the latter was not required for this study. Thus, it was concluded that FLUENT was more suitable for use in the simulations for this study.

However, in the present version FLUENT 6.3.26, it does not appear that Favre averaging is completed. Instead, for variable density flow, Equations 4.5, 4.6 and 4.11 are "interpreted as Favre-averaged Navier-Stokes equations, with the velocities representing

mass averaged values" Ansys (2006a). It is therefore not clear whether the actual process of mass averaging is performed. The flow properties seem to be simply calculated as with incompressible flows, but with variable density as determined from the perfect gas law, and the results simply represent mass averaged values. Therefore, FLUENT 6.3.26 appears to use the same forms of the mass, momentum, and energy equations for both compressible and incompressible flows, although with variable density in compressible flow cases.

\subsection{Turbulence Modelling}

The use of Reynolds or Favre averaging introduces additional unknowns, Reynolds stresses, which may be treated as additional stresses that arise from the randomness of the flow. Solving the Reynolds Averaged Navier Stokes (RANS) equations or Favre Averaged Navier Stokes (FANS) equations requires the use of turbulence models to account 
for the Reynolds stresses. Such models can be found in the forms of algebraic or differential equations, and are generally derived by direct experimental input or by trial-and-error adjustment. No available RANS/FANS model can be used to solve all types of turbulent flows, because in order to get the optimized prediction of the flow field, there is a need to adjust the model's empirical coefficients (Bradshaw (1997)). Turbulence models have found successful applications in engineering design and analysis, even though there is no universal RANS/FANS model for all applications. Compared to the alternative approaches of LES and DNS, RANS/FANS simulations have the advantage of reduced computational time and resource requirements.

RANS/FANS models have been developed to incorporate non-local and flow history effects in the eddy viscosity. The simplest turbulence models introduce a single additional relationship for the turbulent kinetic energy per unit mass $k$. Depending on how they achieve the closure of the turbulent kinetic energy equation, turbulence models can be classified into two categories. The first category includes the turbulent viscosity models, which employ the gradient transport assumption, proposed by Boussinesq (1877):

$$
-\rho\left\{u_{i}^{\prime \prime} u_{j}^{\prime \prime}\right\}=\mu_{t}\left(\frac{\partial u_{i}}{\partial x_{j}}+\frac{\partial u_{j}}{\partial x_{i}}\right)-\frac{2}{3}\left(\rho k+\mu_{t} \frac{\partial u_{i}}{\partial x_{i}}\right) \delta_{i j}
$$

This category is further subdivided into one-equation and two-equation models.

The second category includes the Reynolds stress models (RSM), which determine the turbulent shear stresses by solving transport equations for each stress component. This entails 7 equations for a two-dimensional isothermal flow, and 15 equations for a threedimensional non-isothermal flow. 
FLUENT 6.3.26 has ten available turbulence models, among which five were selected for comparison. These include the Spalart-Allmaras model (S-A), the RNG (Renormalization group) $k-\varepsilon$ turbulence model, the realizable $k-\varepsilon$ turbulence model, the shear stress turbulence (SST) model, and the Reynolds stress model (RSM).

\subsubsection{Spalart-Allmaras (S-A) model}

One-equation models have shown limited capabilities in their predictive ability because the turbulent length scale, which is strongly dependent on the flow configuration and thus not universal in three-dimensional turbulent flows, is determined empirically (Speziale (1996)). The one-equation model of Spalart and Allmaras (1992), which determines the turbulent viscosity directly from a single transport equation, has shown better performance on airfoil and wing applications than the standard one-equation model (Blazek (2005)), because the former does not have the length scale input requirement of the latter. However, Bardina et al. (1997) notes that the S-A model is unable to accurately predict the spreading rates of plane and round jets.

\subsubsection{Standard $k-\varepsilon$ model}

Two-equation turbulence models are the most commonly used in industrial applications, the most popular of which is the standard $k-\varepsilon$ turbulence model (Launder and Spalding (1974)). The standard $k-\varepsilon$ model requires two partial differential equations, one for the turbulence kinetic energy $k$ and another for the turbulence dissipation rate $\varepsilon$, in order to determine the turbulence viscosity, which then leads to the Reynolds stresses through Boussinesq's relation. This model is widely used in industrial simulations because 
of its numerical stability, simplicity, and overall reliability of the solutions (Menter (1991)). This model is also known to have a low accuracy when applied to simulations of boundary layers with adverse pressure gradients (Bradshaw (1997)). Moreover, the standard $k-\varepsilon$ turbulence model has difficulty integrating through the viscous sublayer and requires a viscous correction to reproduce the law of the wall for incompressible flat-plate boundary layers (Wilcox (2000)).

\subsubsection{RNG $k-\varepsilon$ model}

The performance of standard two-equation turbulence models is fairly good at flows of high Reynolds, in which the turbulence is nearly homogeneous and turbulence production is nearly balanced by dissipation. At relatively low Reynolds numbers, however, the turbulent kinetic energy production and dissipation rates may be quite different, which would necessitate an ad hoc adjustment of each empirical coefficient and the turbulent Prandtl number in the standard turbulence model. The RNG $k-\varepsilon$ turbulence model aims to avoid such adjustments by implementing additional equations based on renormalization group theory, which allow for the analysis of changes in turbulent flow at different length scales (Yakhot and Orszag (1986)). Consequently, the RNG $k-\varepsilon$ turbulence model has been shown to perform well in highly strained flows and swirling flows.

\subsubsection{Realizable $k-\varepsilon$ model}

As indicated by the term realizable, this turbulence model partially accounts for turbulent flow physics by following constraints placed on the Reynolds stresses (Shih et al. (1994)). The realizable $k-\varepsilon$ model determines the turbulent viscosity with the use of a new 
function and the dissipation rate by a transport function which is derived from the dynamic fluctuation of mean-squared vorticity (Shih et al. (1994)). This results in a turbulence model which more accurately determines the flow field in flows with rotation, boundary layers in adverse pressure gradients, and separation and recirculation zones (Shih et al. (1994)).

\subsubsection{Shear Stress Transport (SST) model}

The second most widely used two-equation model is the $k-\omega$ model, where $\omega \propto \varepsilon / k$ is a characteristic frequency of the large eddies (Wilcox (2000)). A transport equation for $\omega$ is solved and the turbulent viscosity is then determined as a function of turbulent kinetic energy and dissipation rate. Developed by Wilcox (2000), the $k-\omega$ model has the advantages of numerical stability, simplicity, and more accurate simulation of the viscous sublayer. The simplicity of this model is due to the fact that it requires no damping function (Menter (1994)); instead, one may use Dirichlet boundary conditions at the wall. In addition, it has been shown that, in the logarithmic region of a turbulent boundary layer, the $k-\omega$ model is superior to the $k-\varepsilon$ model when adverse pressure gradients exist and when the flow is compressible (Menter (1992), Menter (1994)). Therefore, the $k-\omega$ model is used in the viscous sublayer and logarithmic regions of the boundary layer. However, the $k-\omega$ model has a strong dependence on the free stream value of $\omega$. The eddy viscosity in the boundary layer has been found to change by more than $100 \%$ if the free stream value of $\omega$ is reduced (Menter (1991)).

Many two-equation turbulence models suffer from an inability to correctly model flows with an adverse pressure gradient or a recirculation zone. The goal of the SST model, 
developed by Menter (1994), is to eliminate this problem. This is accomplished by gradually blending the $k-\omega$ model in the near-wall region and the high Reynolds number $k-\varepsilon$ model in the core flow region, and then modelling the transport of turbulent shear stress similarly to the Johnson-King (JK) turbulence model (Menter (1994)).

Previous two-equation models do not account for turbulent shear stress transport, and determine the turbulent shear stress $\tau_{t}$ by (Menter (1994))

$$
\tau_{t}=\rho \sqrt{\frac{\operatorname{Production}_{k}}{\text { Dissipation }_{k}}} a_{1} k
$$

where $a_{1}$ is a constant. The ratio of production and dissipation of turbulent kinetic energy has been shown to be much greater than 1 in some flows with adverse pressure gradients (Menter (1992), Blazek (2005)). The previous equation would then lead to an overprediction of turbulent shear stress, which results in overestimation of the turbulent (eddy) viscosity. As a result, predictions show that a flow tends to adhere to walls in situations at which it would actually separate. The SST model eliminates this problem with the application of the following equation for the eddy viscosity (Menter (1994))

$$
\nu_{t}=\frac{a_{1} k}{\max \left(a_{1} \omega, \frac{\partial u}{\partial y} G\right)}
$$

The coefficient $G$ is taken to be 0 in free shear flows and 1 in boundary layer flows. Thus, in free shear regions, the relationship for the turbulent eddy viscosity reverts to the expression used by previous two-equation turbulence models.

The SST model has been found to be the most suited turbulence model for turbomachinery flows, due in large part to its accurate prediction of separation and flows with 
adverse pressure gradients. In addition, the improved accuracy comes with little computational cost. Although the Reynolds Stress Model is somewhat more accurate, its higher computational cost and potential instability often outweigh any possible benefits. Therefore, the SST model was chosen for use in the current study.

\subsubsection{Reynolds Stress Model (RSM)}

Departing from the previous two-equation models, the RSM model determines the turbulent stresses by solving a transport equation for each stress component, eliminating reliance on the Boussinesq eddy-viscosity approximation. Unlike two-equation and oneequation models, the RSM accounts for flow history effects through terms representing the convection and diffusion of shear stress tensors. The effects of streamline curvature, system rotation and stratification can be taken into account through convection and production terms (Wilcox (2000)). The RSM model also uses modelled terms, derived from a combination of theoretical assumptions and empirical results. An important term modelled in the RSM is that of pressure-strain, which plays an important role in determining the structure of turbulent flows, distributing turbulent energy among the Reynolds stress components, and accounting for flow anisotropy. This term was initially modelled by considering homogeneous flows, but different approaches to modelling the pressure-strain term have recently been attempted. The coefficients of the RSM may need careful fine tuning in order to avoid solution divergence. The complex Reynolds stress models are known to give results superior to those of one- and two-equation turbulence models for flows with streamline curvature, sudden change in strain rate, and secondary motions of the second kind, although at the cost of increased computing time (Bradshaw (1997)). 


\subsection{Discretization Schemes}

\subsubsection{Grid Selection and Spatial Discretization}

The process of spatial discretization is important in determining the accuracy of the simulation. If regions with high flow variable gradients are not refined to a sufficient degree, significant inaccuracies in the solution are likely to occur, which may lead to solution divergence in some cases. Therefore, substantial effort must be dedicated to the spatial discretization of the model.

There are many grid generation programs commercially available, spanning several specialties. In the present work, the model was meshed with the commercial software packages GAMBIT 2.3.16 and TGrid 4.0.24. GAMBIT 2.3.16 is a surface and volume meshing program, whereas TGrid 4.0.24 is strictly a volume meshing program, but TGrid 4.0.24 contains a more robust prism boundary layer mesh generator than GAMBIT 2.3.16. To optimize the mesh, the surface mesh for the domain was generated in GAMBIT 2.3.16 and then imported into TGrid 4.0.24 for volume grid generation.

The main grid types used in modelling gas turbine flows include $H$-type structured grids and tetrahedral unstructured grids. An intermediate type is a hybrid grid, which uses structured grids in the boundary layer regions and unstructured grids elsewhere.

Structured grids use quadrilaterals to form the surface mesh and hexahedra to form the volume mesh. These grids typically take a long time to generate using algebraic grid generation or grid generation using PDEs (Blazek (2005)), and make mesh adaption (the term adaption is used in FLUENT 6.3.26 documentation instead of the more widely used term adaptation) rather difficult to implement. However, the solution time on structured 
grids is lower, when compared to that for unstructured grids. One major disadvantage of structured meshes is that the physical space must be mapped onto the computational space. This may require that the physical space be altered by splitting or sectioning the domain so as to make it "mappable". For a surface to be "mappable", it must be composed of four or more faces (Ansys (2006b)). If a surface is composed of more than four faces, the additional faces must be combined until the surface contains four faces. This can be seen in Figure 4.1, where the suction and pressure surface sides of the blade are each combined with the attached periodic boundary, upstream and downstream of the blade, to form one face. Therefore, the H-type structured grid is formed in a mappable surface with four sides; at the inlet, at the outlet, one formed by the suction side of the blade and two periodic boundaries and one formed by the pressure side of the blade and two periodic boundaries. It can thus be very difficult to generate a structured grid when the model geometry is complex, as in the case of a blade with a large twist, a non-uniform profile, or a tip gap region. In some cases, the domain can be subdivided into smaller mappable regions, but this can be time consuming and difficult to accomplish. Another disadvantage of structured meshes is that refinement in regions where it is required may lead to unwanted mesh refinement in other parts of the domain, as seen in Figure 4.1. This figure shows that local refinement near the leading edge and the trailing edge of the rotor propagates into the domain, where refinement is unnecessary. In summary, enforcing local mesh refinement in desirable regions only can prove quite difficult.

Unstructured grids are composed of triangular elements on the surface of the model and tetrahedral elements in the volume. The main advantages of this type of grid 


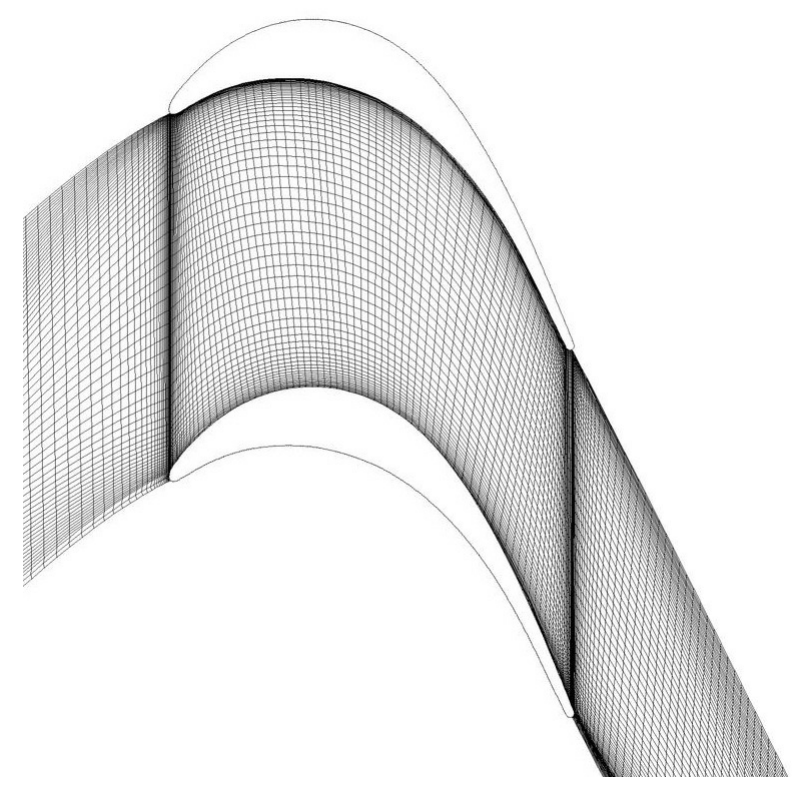

Figure 4.1: Example of a 2D geometry meshed using an H-type structured grid, from Lastiwka (2008).

are the simplicity of discretizing complex domains and that meshing can be done very quickly. However, as the mesh has no inherent order, accuracy in the wall regions is reduced. Achieving the required mesh resolution to decrease errors in the near wall region can lead to extremely large meshes. In many cases, it is worth investing time into the development of a hybrid mesh, as it would yield a smaller node count with respect to an unstructured mesh, thus reducing the need for large computational resources. Unlike the structured grids, unstructured grids cannot be mapped onto a computational space. Therefore, unstructured grids require a connectivity matrix to inform the solver of which nodes are neighbours. This requires additional computational memory and can lead to increased computational time with respect to structured grids of similar density. However, a distinct superiority of unstructured meshes, over structured meshes, is that they can easily be refined locally with 


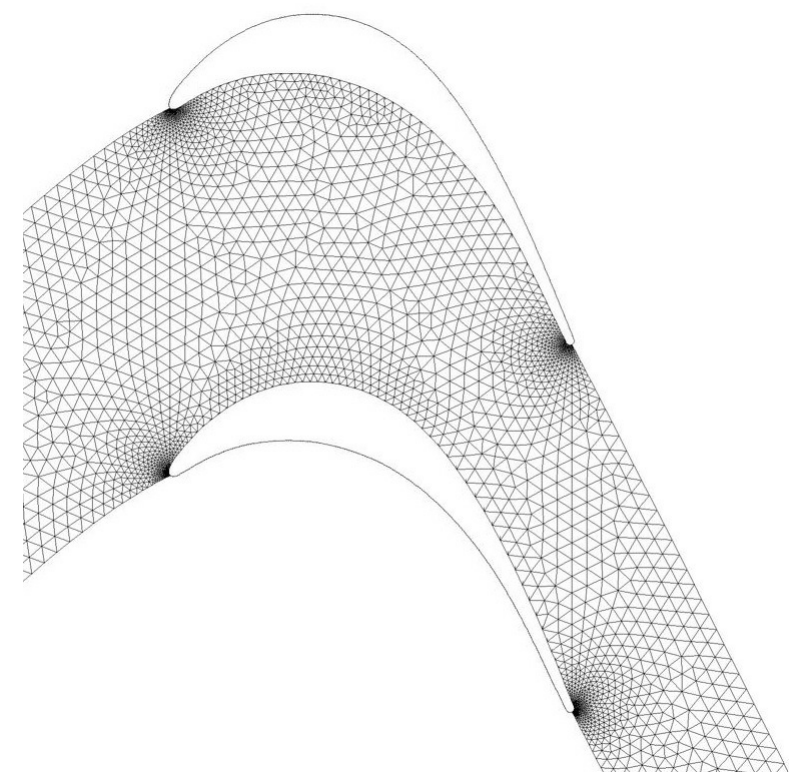

Figure 4.2: Two-dimensional example of an unstructured tetrahedral grid, from Lastiwka (2008).

little effect on the rest of the domain, as shown in Figure 4.2.

An advantageous combination of the unstructured mesh and the structure mesh is the hybrid mesh. This type of mesh allows control of the mesh in the near-wall regions, while also allowing easy meshing of complex geometries. In addition, it does not allow local refinement to propagate into the domain and does not require an excessive number of nodes in the boundary layer to achieve the desired dense meshing near the wall.

The generation of a structured mesh in a 3D gas turbine requires specialized meshing software. However, for 2D simulations, a hybrid mesh (Figure 4.3) can be constructed using only GAMBIT 2.3.16. GAMBIT 2.3.16 has the ability to generate an unstructured mesh and a 2D structured mesh in the boundary layer simultaneously. For the 3D simulations, an unstructured mesh was first generated using GAMBIT 2.3.16 and TGrid 4.0.24 


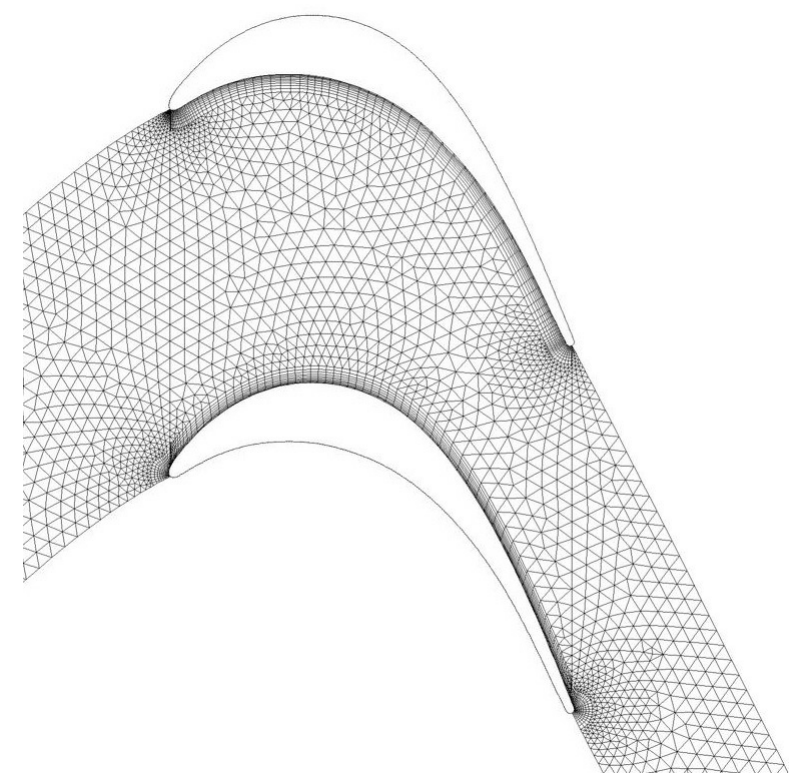

Figure 4.3: A hybrid mesh with structured cells near wall regions and unstructured cells elsewhere; from Lastiwka (2008).

was used to generate the 3D structured mesh in the boundary layer after the unstructured mesh was created.

As one of the goals of this study is to achieve results of fairly high accuracy, it was desirable to avoid the artificial diffusion associated with first-order spatial discretization (Barth and Jespersen (1989)). A second-order upwind scheme was used for spatial discretization of all flow variables, and the Green-Gauss Node-Based method calculated the required gradients and derivatives. 


\subsubsection{Temporal Discretization}

The Courant-Friedrichs-Lewy number $(\mathrm{CFL}=u \Delta t / \Delta x)$ is the ratio of the time step (that is, the internal solver time step between solution iterations at a given time) and the cell residence time. As it affects both the rate of convergence and the stability of the simulation, the CFL number is generally set to a low initial value, such as 0.0001 . This is then increased gradually, in order to maintain both stability and convergence rate, until sufficient convergence is obtained. Experience showed that simulations became unstable when CFL > 0.1, if the explicit solver were used, and when CFL > 1, if the implicit solver were used. However, to sustain the stability of the simulation one should use slightly lower values than the limits of the previous inequalities.

For the unsteady simulations, a second-order density-based scheme was used for time discretization, as this scheme is known to be unconditionally stable. As the implicit formulation of the density-based solver was used, FLUENT 6.3.26 employed a second-order Euler backward differencing scheme for the dual-time formulation using a preconditioned pseudo-time derivative for more accurate unsteady results. FLUENT also used an explicit pseudo-time marching approach for the inner iterations (iterations within each time step), as well as a three-stage Runge-Kutta scheme. Note that the solver's implicit formulation computes all flow variables in all cells simultaneously.

In order to help accelerate the convergence of the simulations, the Algebraic Multigrid (AMG) algorithm was implemented by FLUENT. It is known to be beneficial for unstructured meshes, because no coarse grids are generated or stored, thus reducing computing time. The alternative Full-Approximation Storage (FAS) multigrid method has been found 
to greatly improve convergence rates when non-linearities are present due to non-linearities in the system being carried down to the coarse levels through re-discretization (Ansys (2006a)). However, the use of explicit time-stepping in this study prohibits the use of FAS multigrid, as the temporal accuracy of the fine grid solution would be compromised.

\subsection{Domain Scaling}

A common approach in computational fluid dynamics is to take advantage of existing geometric periodicity in order to reduce computer memory, storage space and computational time requirements. In such cases, only one of the repeated sections of the domain is considered and periodic boundary conditions are applied at its boundaries. In the case of turbine stages, which consist of successive rows of stator vanes and rotor blades, advantageous geometric periodicity would require a low-integer ratio of rotor blades to stator vanes. However, common design practice is to use blade-to-vane ratios of large integers to help prevent specific modes of vibration. Thus, accurate simulation of these turbine stages would require consideration of the entire stator and rotor. To circumvent this problem, gas turbine analysts have used several different methods, as indicated by Mårtensson et al. (2003), including rotor blade scaling, phase lagged periodic boundary conditions, and time inclined boundary conditions.

The domain scaling method used in this study is based on the method developed by Rai (1987) and Rai and Madavan (1990). The rotor blade geometry is scaled such that a constant solidity ratio is maintained (constant ratio between chord and pitch). The changing

pitch results in the addition or subtraction of blades (with a constant rotor circumference), 
allowing the user to achieve a low-integer blade-to-vane ratio. This method has been found to yield some disparities in various aspects of turbomachinery flow, such as blade surface pressure distribution, passage vortices, and pressure losses (Lastiwka (2008)). This should be of little consequence for the present study, however, which focuses on comparisons of cases with identical geometries. This method of scaling to reduce the domain was also used by Gundy-Burlet and Dorney (2000), and Sondak and Dorney (2000).

For a comprehensive review of the domain scaling method used in this study, see Lastiwka (2008). 


\section{Chapter 5}

\section{Inlet Temperature Specification}

This chapter outlines the procedure used for determining appropriate inlet temperature fields for specification as inlet boundary conditions. This should be helpful in understanding the simulation conditions and preliminary procedures.

\subsection{Definition of Inlet Temperature Field}

At the inlet of a turbine section the flow is unsteady and turbulent, with properties highly dependent on the combustion process, and, to a lesser degree, the compression in the compressor section and the ambient air conditions. For the purposes of this study, the inlet temperature will be specified as steady and with an azimuthal periodicity which has the same period as the computational domain. 


\subsubsection{Spanwise Profile}

A set of inlet total temperature values were supplied by the manufacturer from the turbine considered in this study. These consisted of total temperatures at different spanwise locations of the inlet surface and were taken to represent circumferential averages over the entire inlet cross-section. As shown in Figure 5.1, the total temperature is relatively low at the endwalls, rising smoothly to a peak total temperature at a spanwise location that was approximately at $75 \%$ of the span from the hub endwall (located at a spanwise coordinate of 0.0 in the figure) to the casing endwall (located at a spanwise coordinate of 1.0 in the figure). These data were measured using available probes, and the values at the endpoints do not match accurately the total temperatures at the surfaces of the endwalls. However, we were advised by the manufacturer that approximating the endwall temperatures by the given values should not have a significant effect on the results of this study.

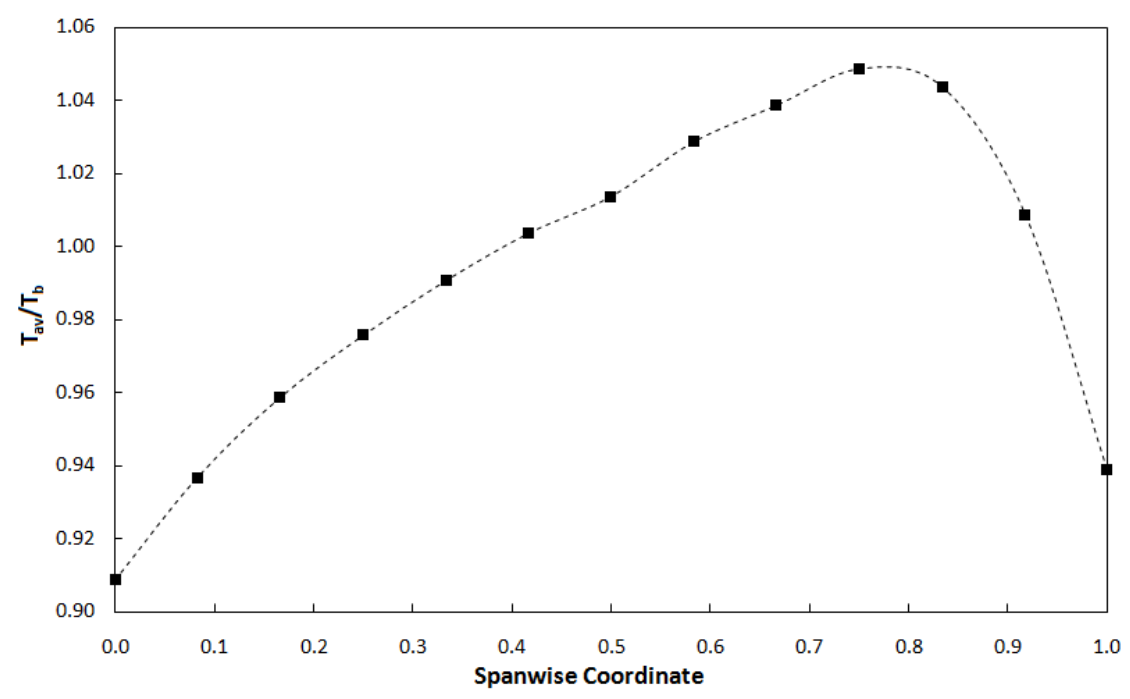

Figure 5.1: Circumferentially-averaged spanwise profile of total temperature at the inlet, normalized by the mass-weighted bulk total temperature across the inlet $T_{b}$; the value of $T_{b}$ has been withheld according to the non-disclosure agreement with the industrial sponsor. 
Profiles of total temperature at the inlet of high pressure turbines have been presented by Povey et al. (2003) and Qingjun et al. (2007) (see Figure 5.2). The relative location of peak total temperature in Figure 5.1 differs from those in previous studies, in which the peak is located close to midspan. Instead of the more commonly used straight duct between the combustors and the turbine, the engine of present interest has a reverseflow combustor design followed by a highly curved transition duct, which redirects the flow by approximately $180^{\circ}$ (see Fig. 1.2). Centrifugal forces in the curved duct tend to shift the lower-density hot fluid from midspan towards the casing wall, thus skewing the temperature profile. Additionally, the total temperature in the present turbine inlet is slightly higher near the casing endwall than near the hub endwall. This difference is also attributed to the specific transition duct geometry. The small-radius curvature of the casing endwall provides less surface area for convective losses, as well as being adjacent to a high-temperature region. The hub endwall, in contrast, has a larger surface area and is adjacent to a region of lower temperature.

\subsubsection{Circumferential Variation}

In order to represent the turbine inlet conditions more realistically, we modulated the circumferentially-average spanwise profile provided by the manufacturer to an inlet field that also has circumferential variation. The inlet field of a turbine typically varies circumferentially, with peaks corresponding to the relative locations of discrete combustors and valleys to locations in-between. As a constraint in this transformation, we required that the same circumferential average be maintained at each spanwise location. This suggested the application of a sinusoidal modulation to vary the spanwise total temperature profile 


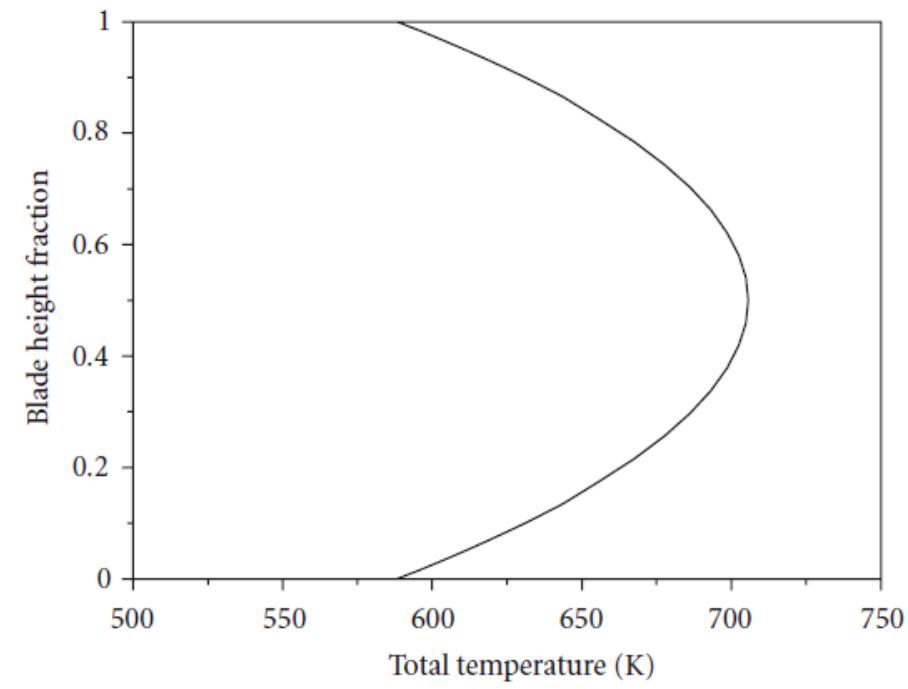

Figure 5.2: Typical radial profile of turbine inlet total temperature (Qingjun et al., 2007) around the circumference of the inlet.

The periodicity of the numerical simulation was maintained by selecting a sinusoidal period that was equal to the circumferential period of the simulation geometry. In this way, each peak in total temperature represented a single hot streak coming out of a combustor element and corresponded to a single stator vane. This choice has been approved by the manufacturer as consistent with realistic conditions. The modulating equation for the inlet total temperature is

$$
T(z, \varphi)=T_{a v}(z)+0.1 T_{b}\left[\frac{T_{a v}(z)-T_{l i n}(z)}{T_{a v}\left(z_{m}\right)-T_{l i n}\left(z_{m}\right)}\right] \sin \left(N \varphi+\varphi_{o}\right)
$$

where

- $T_{a v}(z)$ is the specified average total temperature for a given spanwise location $z$

- $T_{l i n}(z)$ is a straight line connecting the total temperatures of the endwalls 
- $z_{m}$ is the spanwise location of the circumferentially-averaged total temperature peak

- $T_{b}$ is the mass-weighted bulk total temperature across the inlet

- $N$ is the number of periodically repeating domains comprising the entire turbine

- $\varphi$ is the circumferential coordinate

- $\varphi_{o}$ is a phase shift used to position the hot streaks with respect to the stator vanes.

Note that the term within square brackets in Equation 5.1 varies from zero at the inlet walls to unity at the point of peak average temperature, where the difference between maximum and minimum temperatures is greatest. This restriction allows for the desired amplitude of variation near the hot streak's peak, while maintaining consistent temperatures along the endwalls. The maximum variation from the provided average values has been set at $\pm 10 \%$ of the inlet's mass-weighted bulk total temperature. This ensures that the total temperature of the fluid near the endwalls increases with distance from the walls, as is expected by considering the turbine cooling systems.

The minimum and maximum extremes of the derived total temperature field are shown in Figure 5.3, along with the average profile supplied by the manufacturer. The maximum curve represents the total temperature profile at the circumferential location of the hot streak, while the minimum curve is characteristic of the location between hot streaks.

The resulting two-dimensional field of total temperature for a single geometric period is shown in Figure 5.4. The specified inlet conditions are intended to model the significant circumferential variations produced by discrete combustors and related geometry. 


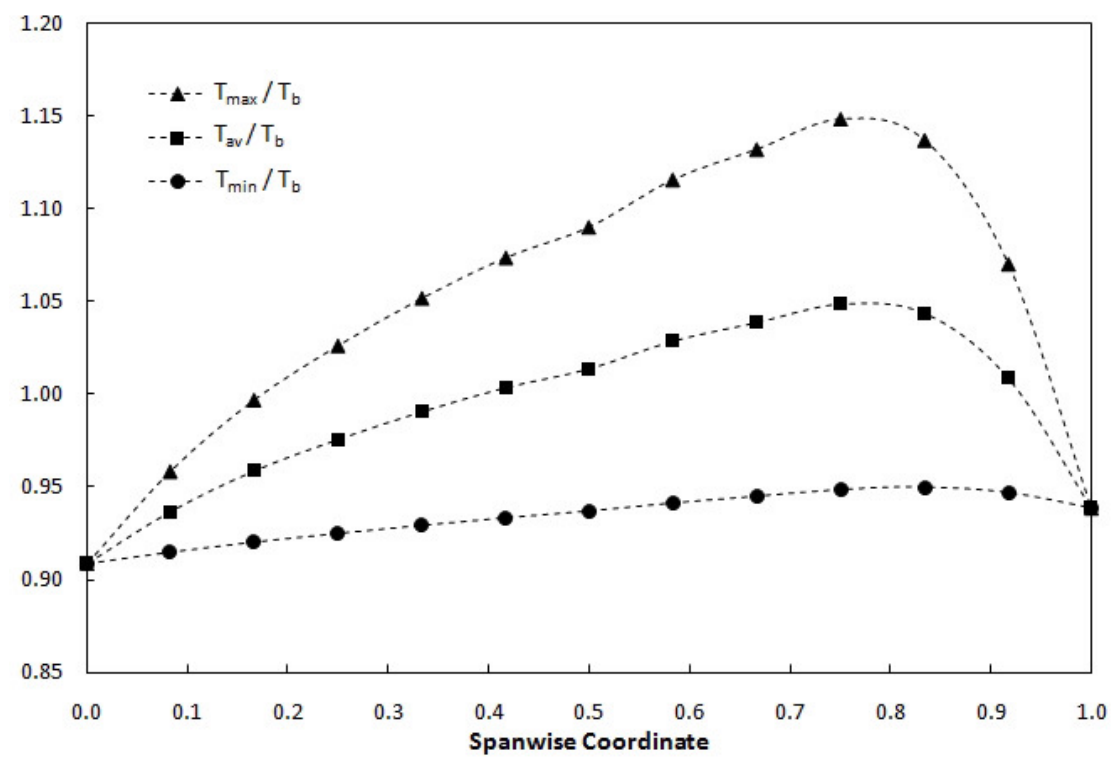

Figure 5.3: Original and extreme spanwise profiles of total temperature at the inlet, normalized by $T_{b}$.

The resulting temperature contours are largely dependent on dilution between gases of different properties as they flow from the combustor into the turbine. Similar trends have been observed by Povey et al. (2003) and Li et al. (2006), among others, with varying degrees of dilution and attenuation. The manufacturer has also verified that Figure 5.4 represents a fairly accurate approximation of realistic turbine inlet conditions.

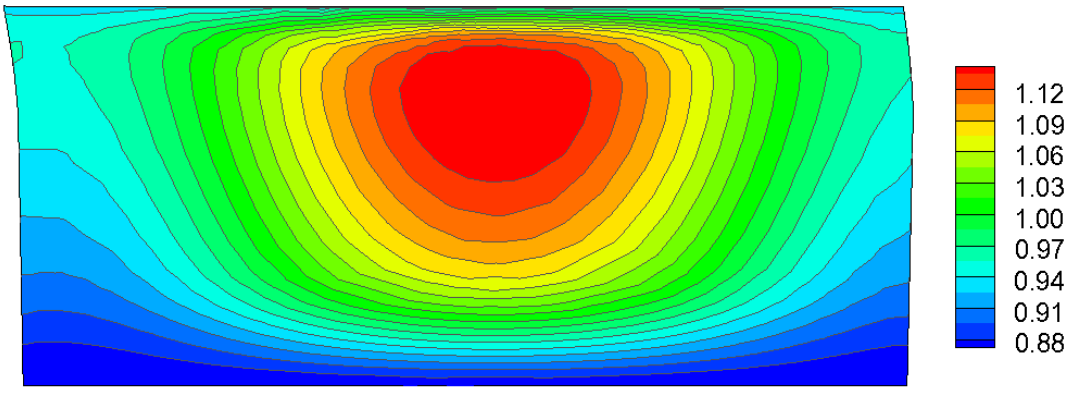

Figure 5.4: Contours of total temperature on the inlet, normalized by $T_{b}$. Dotted line indicates downstream position of vane leading edge. 


\subsection{Circumferential Positioning of Hot Streaks}

For cases involving non-integer ratios of combustor igniters (or similar features) to stator vanes, the resulting hot streak pattern will interact with different locations on the stator vanes, requiring simulation of the entire turbine stage in order to determine the full effect of the temperature profile on the operation of the turbine. In the case of integer ratio geometry, the hot streaks can be clocked or aligned with respect to the stator vanes to determine the optimum geometry for turbine performance for the operating conditions. For our choice of a ratio of 1 , the simulation of only a single geometrically periodic section is required, as the alignment of each hot streak and a corresponding stator vane is identical for all hot streaks.

Although geometric alignment of the inlet field is quite simple (see Figure 5.4), it may not result in the desired impingement on the stator vane leading edge further downstream. The rotation of the upstream flow field induced by the stator is of sufficient strength to deflect the hot streak by nearly a quarter of the periodic circumferential domain, toward the suction side of the corresponding stator vane. This is illustrated in Figure 5.5, which shows flow pathlines originating near the spanwise location of peak hot streak total temperature. A circumferential phase shift of the inlet field alignment is therefore necessary in order to achieve the desired downstream alignment between the hot streak and the vane leading edge.

In one of the two configurations of interest, the hot streak was aligned such as to impinge onto the leading edge of the vane, so that it was effectively split by the vane. This configuration will be referred to as the vane-impinging (VI) case. In the other configura- 


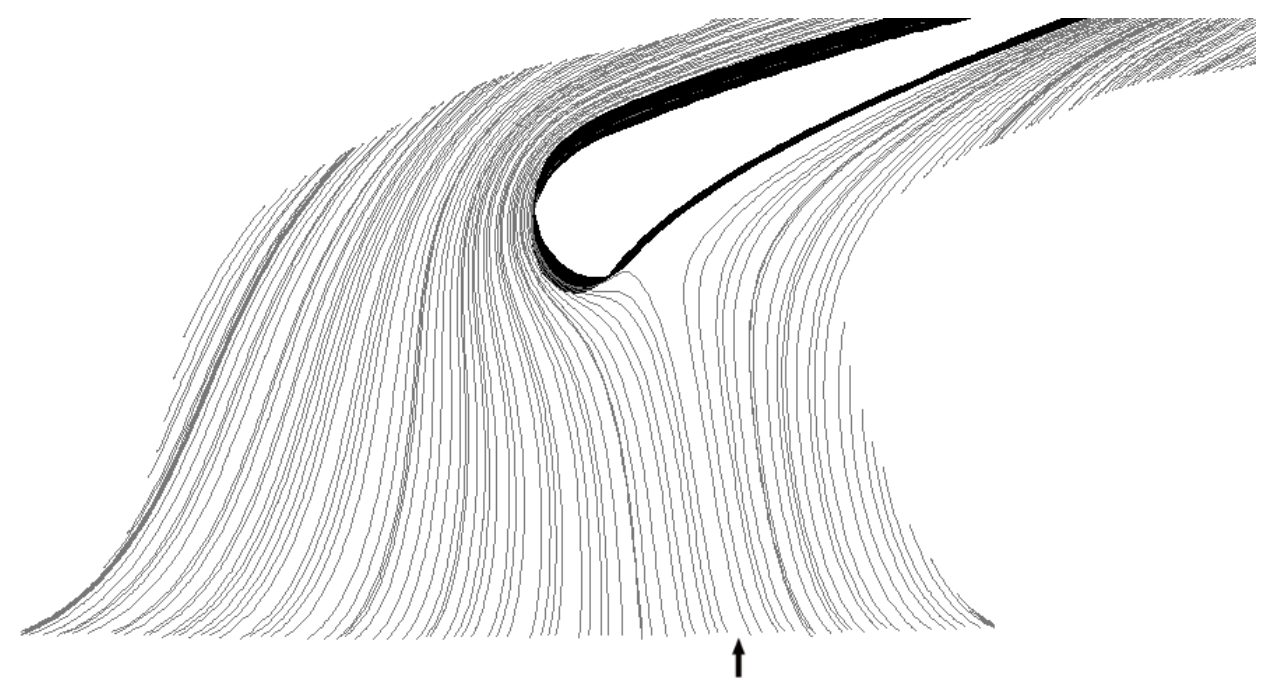

Figure 5.5: Flow pathlines approaching stator vane. Arrow indicates adjusted location of aligned hot streak.

tion, the hot streak passed through the middle of the passage between stator vanes. This configuration will be referred to here as the mid-pitch (MP) case. This allows the hot streak to interact directly with the rotor blades, as it is largely unchanged by the stator section. These two configurations are deemed to be the extreme cases of turbine performance, as far as hot streak alignment, in isolation of other effects, is concerned. Similar configurations have been examined by previous authors.

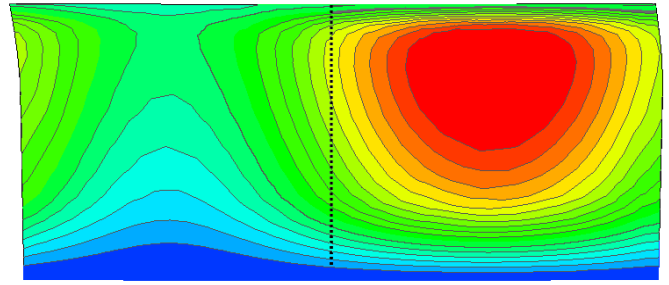

(a)

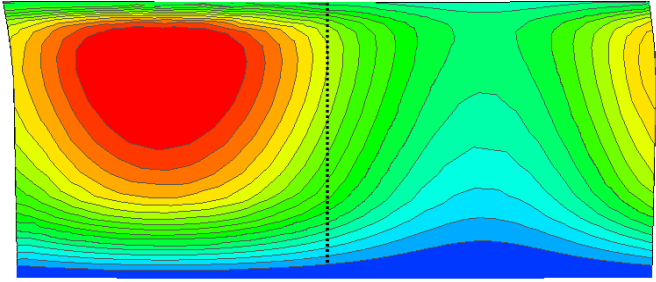

(b)

Figure 5.6: Contours of inlet total temperature, normalized by $T_{b}$, for the (a) $V I$ and (b) $M P$ configurations. Dotted lines indicate downstream position of vane leading edge. 


\subsection{Implementation}

The total temperature field must be applied as the thermal condition for the inlet boundary of the computational domain. Fluent 6.3 .26 provides a simple method of specifying non-uniform boundary conditions. A program in $C$ language must be used as a User-Defined Function (UDF) to provide the code with the desired temperature variation by specifying a value for each queried location, depending on the parameters written into the program (such as an equation or interpolated table of values).

For the purposes of this study, it was decided to use the compiled method of defining a UDF. The program is built into an object code library in the same way as the Fluent executable code itself, allowing Fluent to dynamically load and directly access the program. A compiled UDF is therefore executed faster than an interpreted UDF, which is accessed through an intermediate preprocessor. Interpreted UDFs are also limited in the use of the $C$ language and access to Fluent data, though they are more portable to other platforms and systems.

The program used in this study obtains the position of the current cell from Fluent, then uses quadratic interpolation to determine the circumferentially-averaged total temperature for that spanwise position, based on the manufacturer's data set. The circumferential modulation, as defined by Equation 5.1, is then applied to this average in order to achieve the desired two-dimensional inlet field. This information is then used by Fluent to define the inlet boundary, and can be selected for use when defining boundary conditions. 


\section{Chapter 6}

\section{Numerical Procedures and}

\section{Conditions}

The 3D gas turbine model used in of this study was developed using geometry and inlet specifications provided by the manufacturer. This chapter summarizes the simulation geometry, its discretization, and the numerical conditions and schemes used for the simulation of the turbine.

\subsection{Hardware and Software}

The simulations were performed on four different computer systems: a) an inhouse personal computer (PC), b) an in-house cluster of Sun Fire servers, c) an in-house cluster of Dell servers, and d) the High Performance Computing and Virtual Laboratory (HPCVL). The PC used for preliminary computations and some pre- and post-processing of the data is an HP Pavilion a1730n workstation with a $2.6 \mathrm{GHz}$ AMD Athlon $64 \mathrm{X} 2$ 
Dual Core 5000+ processor and 3 GB of RAM. The local Sun Fire cluster consists of six Sun Fire X2200 M2 x64 servers, each with $2 \times 2.2 \mathrm{GHz}$ AMD Opteron 2214 dual core processors and 8 GB of RAM. The local Dell cluster consists of six PowerEdge SC1435 x64 servers, each with $2 \times 2.6 \mathrm{GHz}$ AMD Opteron 2218 dual core processors and 8 GB of RAM. The resources available at HPCVL consisted of six Sun Fire 25000 nodes, each with $72 \times$ dual-core UltraSPARC-IV+ 1.5 GHz processors and 576 GB of RAM.

Post-processing was performed on an additional in-house personal computer, a Dell Studio 540 workstation with a $2.33 \mathrm{GHz}$ Intel Core 2 Quad Q8200 processor and 8 GB of RAM. In-house data storage and backup resources consisted of a PowerVault MD1000 with $15 \times 500$ GB RAID-controlled hard drives.

The software package GAMBIT 2.3.16 was used for computer model generation and mesh generation. TGrid 4.0 was used for enhanced mesh control and for 3D structured wall meshing. The commercial code FLUENT 6.3.26 was used for all CFD simulations, and Matlab and Tecplot 360 were used for post-processing and visualization.

\subsection{Computational Domain and Grid Generation}

The original turbine stage to be analyzed consisted of a stator with 13 vanes and a rotor with 38 blades. Because the number of rotor blades is not an integral multiple of the number of stator vanes, the numerical analysis would require both large computing resources and long simulation times. To reduce the required time and resources to more practical levels, the simulations were conducted with the rotor section appropriately scaled, using the approach discussed in Section 4.4. Scaling the rotor section increased the number 
of rotor blades from 38 to 39 . The numerical model therefore has a vane count to blade count ratio of $1: 3$, which permitted the implementation of rotationally periodic boundary conditions.

The geometry data obtained by the manufacturer consisted of a section of transition duct, the first stage of a high pressure turbine, and a short outlet duct. The inlet plane was located in the middle of the $180^{\circ}$ transition duct, which connected the combustor with the high-pressure transonic turbine stage. The outlet duct was included in order to facilitate visualization and analysis downstream of the rotor, as well as to avoid possible convergence problems associated with the unsteady simulations and to provide some separation between the rotor and the outlet's reflecting boundary condition (see Section 6.3). The numerical domain used for the simulations is shown in Figure 6.1.

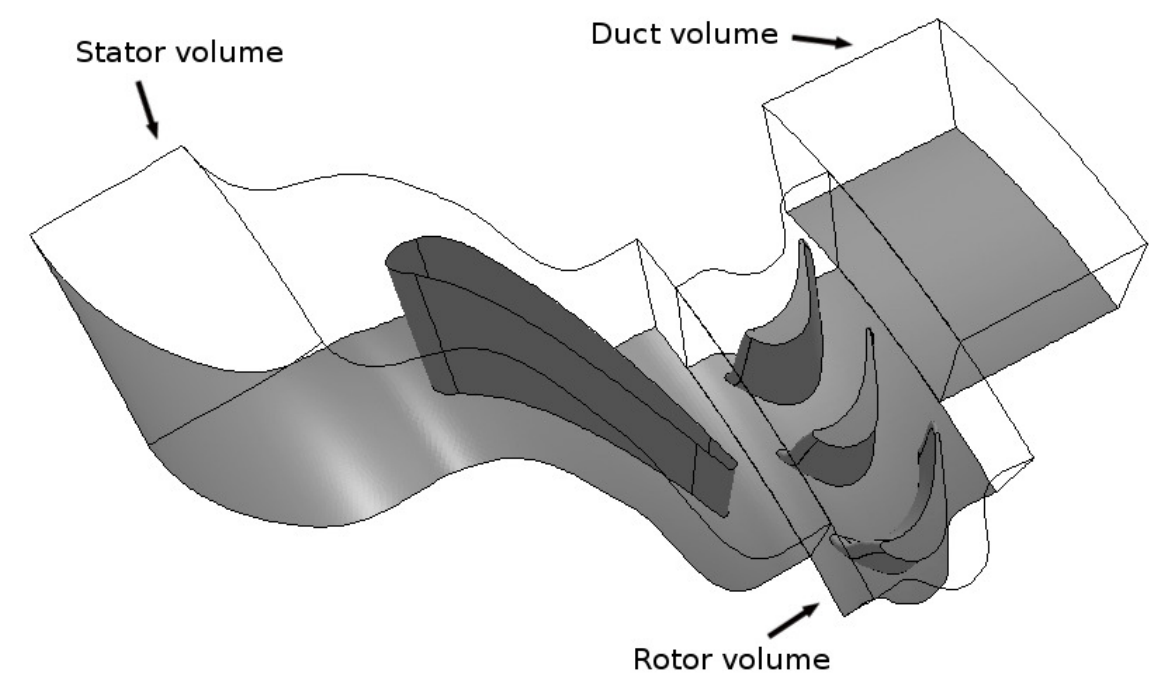

Figure 6.1: Numerical domain, separated into three volumes; the stator and duct volumes are fixed, whereas the rotor volume is rotating.

The numerical domain was split into three separate domains, namely the stator, 
rotor and duct sections. The rotor domain also includes a gap region between the blade tips and the casing. The stator and duct domains were kept fixed, whereas the rotor domain was rotating. The rotational periodicity was determined from the ratio of the number of rotor blades to the number of stator vanes, which for the present scaled model was

$$
\Lambda=\frac{39}{13}=\frac{3}{1}
$$

Accordingly, the period of the numerical geometry contained three rotor blade passages and one stator vane passage. The angle of rotational periodicity can be determined by

$$
\theta_{r p}=\frac{360^{\circ}}{\left(N_{r t} / N_{r t, s i m}\right)}
$$

where $N_{r t}$ and $N_{r t, s i m}$ represent the number of rotor blades in the full and periodicallymodelled turbines, respectively. In this case, the rotational periodicity angle was approximately $27.7^{\circ}$. This resulted in a $27.7^{\circ}$ sector for all numerical volumes, or a reduction of the full (scaled) turbomachine to $1 / 13^{\text {th }}$ of its original size.

The numerical domain was created using GAMBIT 2.3.16 and model data provided by the manufacturer. The surface of each blade was generated from 23 profiles from the hub to the tip, and the surface of each vane was constructed from 25 profiles. A hybrid mesh was desired to discretize the turbine's volume in order to achieve the desired resolution near the domain walls. However, GAMBIT 2.3.16 was unable to generate the required 3D structured wall mesh. Therefore, an initial unstructured mesh of tetrahedral elements was generated in GAMBIT. This mesh was imported into TGrid, which generated a 3D structured wall mesh. 
The 3D structured wall mesh was generated by following procedures developed in past studies. TGrid was used to create 5 layers of prism mesh elements over the walls of the model providing the desired resolution. The height of the first layer was $0.007 \mathrm{~mm}$, with a geometric growth rate of 1.3. This resulted in having 10 prism elements across the tip gap. For the remainder of the volumes, an unstructured mesh was used. The mesh density was distributed using size functions such that the density was greater near walls and in the gaps between the blade tips and the rotor casing. Thus, the hybrid mesh is comprised of tetrahedral elements in the core region and prismatic hexahedral elements in the boundary regions. The mesh generated for the numerical domain and used to complete this study is shown in Figure 6.2.

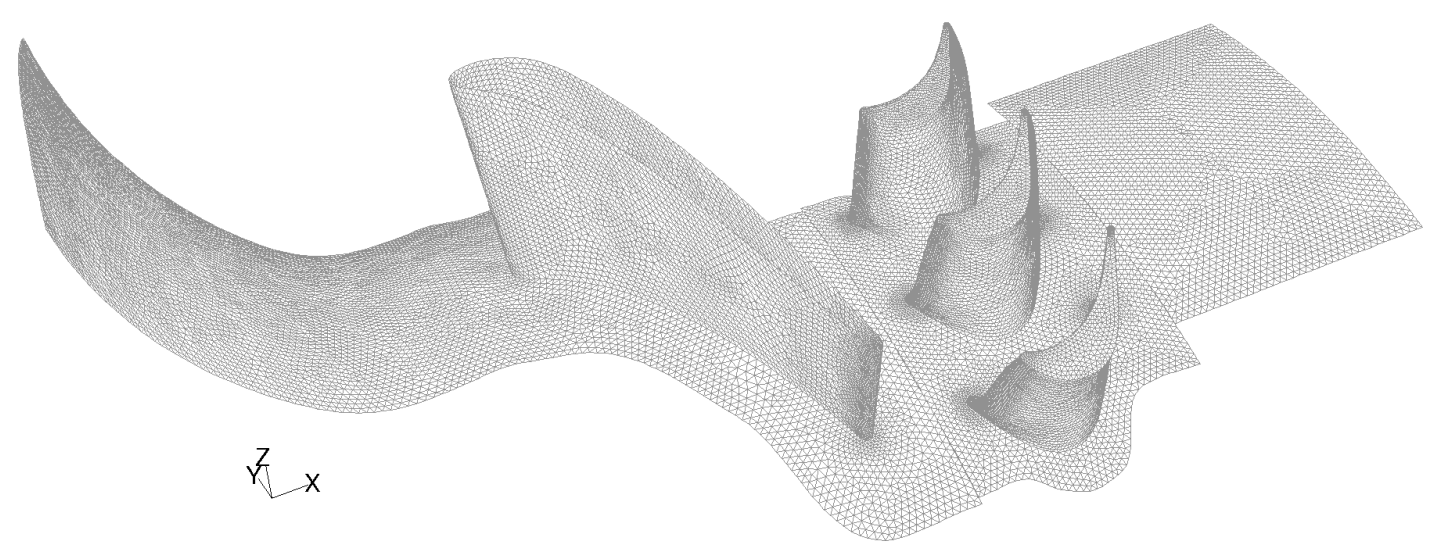

Figure 6.2: Surface mesh for computational grid. 


\subsubsection{Grid Verification}

To reduce the necessary computing time and resources, an initial numerical verification study was conducted on the reference case. This study used the mixing plane approach. The mixing plane model circumferentially averages the flow variables between ordinarily rotating and non-rotating domains, allowing the user to run a steady solver and obtain an approximation of the time-averaged solution. Three different meshes, termed coarse, medium, and fine, were created, with 1.2, 1.7, and 2.2 million cells, respectively.

The total-to-total pressure ratio across the turbine stage and the degree of reaction (defined as the ratio of the static enthalpy change across the rotor to the change across the turbine stage) were computed once simulations with each mesh had converged. The difference between the coarse and the medium meshes was 0.03 , and between the medium and fine meshes it was 0.01. Based on this study, the medium mesh was assessed to provide sufficient accuracy for the computation of mean properties, although this verification was not conclusive concerning the accuracy of prediction of unsteady effects.

A second mesh resolution study was subsequently conducted using the sliding mesh approach (that is, the rotor domain was slid past the stationary domains in a realistic manner, and simulations were conducted using an unsteady solver). Unfortunately, this unsteady study used only the two smaller meshes mentioned previously, as the available resources did not allow for full unsteady simulations using the fine mesh within a realistic time frame. The time-averaged total-to-static pressure ratios across the turbine stage were found to be essentially the same for the two meshes. However, the temporal standard deviation of pressure fluctuation ratios, averaged spatially across the rotor outlet, was $0.06 \%$ 
for the coarse mesh and $0.5 \%$ for the medium mesh. Although this indicates some sensitivity of unsteadiness to the mesh size, the medium mesh with 1.7 million cells was selected for the main simulations, as it combines fairly high accuracy with relatively low computing time. This decision was supported by the turbine's manufacturer, namely their department most experienced in CFD.

\subsection{Simulation Conditions}

In the present study, the interest focuses on the behaviour of a steady-state periodic solution, and disregards the start-up flow field. Therefore, initial conditions did not need to match the actual initial conditions of a real turbine. However, the choice of initial condition is important, as it affects the convergence rate and the overall stability of the simulation. The initial conditions were computed by FLUENT from the conditions set at the inlet boundary. The turbine manufacturer provided data specifying the total pressure and total temperature of both the inlet and outlet boundaries. The specified pressures at the inlet and outlet of the domain were $56 \%$ above and below the operating pressure, respectively. This results in a pressure ratio $\left(P_{\text {in }} / P_{\text {out }}\right)$ of approximately 3.5 . The backflow total temperature at the outlet was set to $76 \%$ of the inlet total temperature. The turbulence was specified at the inlet and for the outlet backflow as well, by specifying the fluid turbulent intensity as $3 \%$ and the hydraulic diameter as $0.48 \mathrm{~m}$ at both boundaries.

To reduce round-off errors, operating pressure for the domain was set at a value equal to the average between the inlet static pressure and the outlet static pressure. Pressure values for inlet and outlet were set as gauge pressures. No-slip and adiabatic conditions 
were applied at the walls of the geometry.

The reflecting boundary condition (RBC) was selected to specify the static pressure at the outlet boundary; this results in a closer match to actual turbine conditions, compared to a non-reflecting boundary. In order to improve the flexibility of the RBC as the outlet condition, the static pressure was specified on the hub line of the outlet plane, rather than on the entire outlet plane, by activating the radial equilibrium relation. The outlet duct domain, with a length of two blade axial chords, was implemented to eliminate any possible interactions of the flow in the rotor with reflected pressure waves generated on the blades at the turbine outlet.

The material properties of the working fluid, specifically the products of combustion, were defined for FLUENT as varying with temperature. The specific heat $\left(\mathbf{C}_{\mathbf{p}}\right)$, thermal conductivity $(\kappa)$, and viscosity $(\mu)$ were determined from cubic polynomials. In modeling the highly-compressible flow, the density was assumed to follow the ideal gas law

$$
\rho=\frac{P}{\frac{\mathbf{R}}{M W} T}
$$

where $\mathbf{R}$ is the gas constant and $M W$ is the molecular weight of the gas. The values of various properties for the working fluid are listed in Table 6.1.

Table 6.1: Gas properties for air as used in simulations

\begin{tabular}{lll}
\hline $\mathbf{C}_{\mathbf{p}}$ & $(\mathrm{J} / \mathrm{kg} \mathrm{K})$ & $942.359 \cdot T+0.1931728 \cdot T^{2}-9.042406 \times 10^{-7} \cdot T^{3}$ \\
$\kappa$ & $(\mathrm{W} / \mathrm{m} \mathrm{K})$ & $0.01234207 \cdot T+5.1994401 \times 10^{-5} \cdot T^{2}+3.580143 \times 10^{-9} \cdot T^{3}$ \\
$\mu$ & $(\mathrm{kg} / \mathrm{ms})$ & $7.058711 \times 10^{-6} \cdot T+4.195762 \times 10^{-8} \cdot T^{2}-6.355986 \times 10^{-12} \cdot T^{3}$ \\
$\mathbf{R}$ & $(\mathrm{J} / \mathrm{K} \mathrm{mol})$ & 8.314 \\
$M W$ & $(\mathrm{~kg} / \mathrm{mol})$ & 28.966 \\
\hline
\end{tabular}

The main objective of this study is to accurately predict the effects of changing inlet temperature fields, in order to apply the findings to actual turbine operation. Therefore, 
the boundary conditions in the simulations were required to match the actual boundary conditions found in a working turbine. Nevertheless, some simplifications and assumptions were made to reduce computational costs.

For the reference case, the temperature was set as constant over the entire inlet. For the two cases with hot streaks, the inlet fields describing the VI (vane-impinging) and MP (mid-pitch) hot streak configurations were used, respectively. Following the substitution principle, as mentioned in Section 3.5.1, the total pressure was uniformly specified over the inlet boundary, to prevent extraneous secondary flow in the stator section.

The model was split into three domains in order to accommodate a rotating rotor, a stationary stator and a stationary duct. All domains were meshed separately and were connected together with a sliding mesh interface (or a mixing plane in some preliminary stages of simulations). The stationary domains were those of the stator and duct, while the rotor domain rotated with an angular velocity equal to that of the actual turbine.

Three periodic boundary sets were defined, one pair for each domain volume. These periodic boundaries were defined as rotational periodic boundaries with an axis of rotation coincident with the turbines axis. Figures 6.3 and 6.4 show the periodic boundaries with respect to the rest of the model.

In order to simulate the motion of the rotor, wall boundaries were assigned specific rotational speeds. The stator and duct domains were given a rotational speed of $0 \mathrm{rad} / \mathrm{s}$, with the associated walls following the respective domains. The rotor domain was assigned a rotational speed equal to that of the design speed of the actual turbine, along with most of the boundaries. The rotor's casing wall boundary was assigned a global rotational speed 


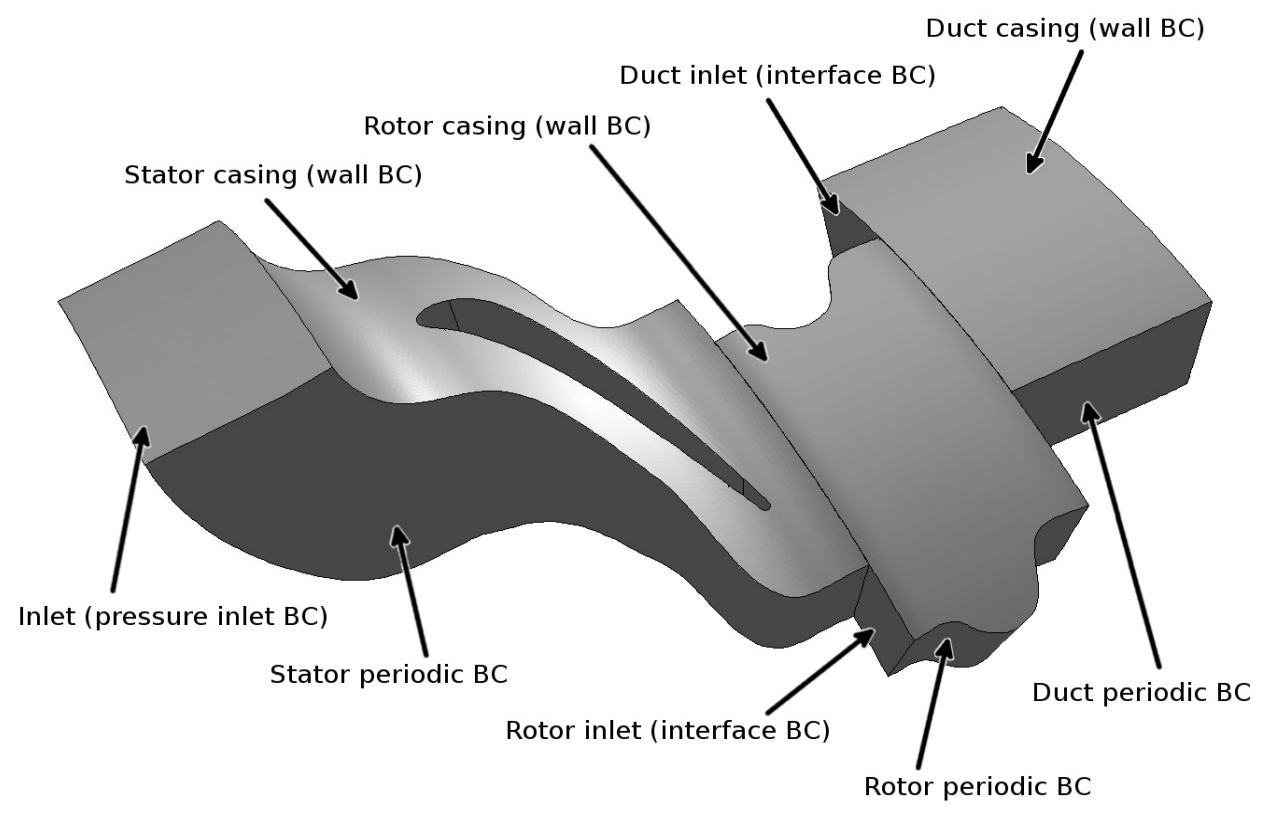

Figure 6.3: Boundary conditions in computational domain.

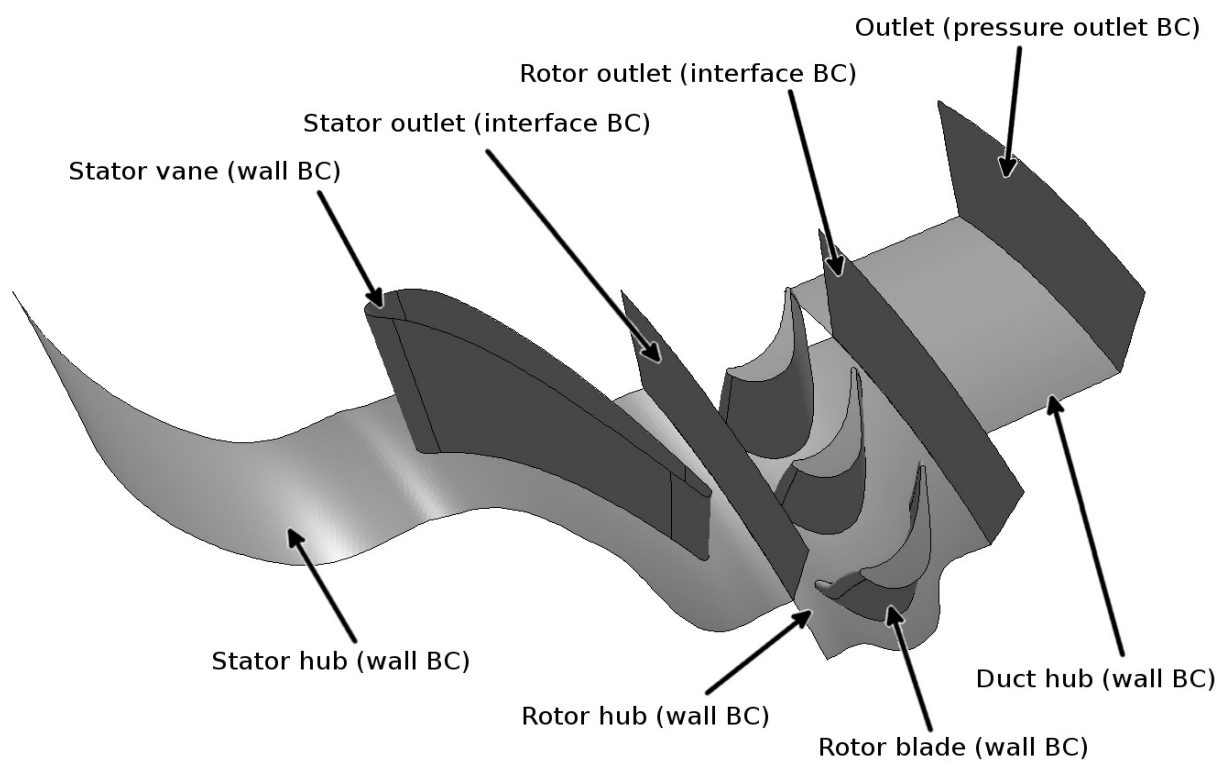

Figure 6.4: Boundary conditions in computational domain. 
of $0 \mathrm{rad} / \mathrm{s}$, to agree with the production engine.

In addition to the previously mentioned conditions, the walls were assigned an energy equation boundary condition. Due to the focus on temperature and its effects, it is important to select appropriate thermal boundary conditions for the surfaces of the stator vanes, rotor blades, and hub and casing endwalls. Sondak and Dorney (2000) found that the use of boundary conditions incorporating a conductive model resulted in surface temperature variations which were significantly lower than those obtained with adiabatic boundaries. The simulated conduction resulted in different temporal variations and timeaveraged temperature results, and would thus be undesirable when time-variant thermal details are required.

Adiabatic boundary conditions were also used by Sondak et al. (2002) and GundyBurlet and Dorney (2000). It is recognized that the use of adiabatic boundaries is not entirely realistic and introduces some error in the calculated temperature distributions for the surfaces. If available, more realistic surface conditions would be preferred, such as constant temperature fields or specified (non-zero) heat flux distributions, as used by Dorney and Davis (1993) for film cooling. However, as noted by He et al. (2004), the use of adiabatic surfaces is not expected to significantly affect the comparative characteristics of thermal loading produced by the distinct hot streak configurations of current interest. All things considered, it was decided to specify adiabatic wall surfaces (zero heat flux) in this study. 


\subsection{Modelling Rotation}

FLUENT 6.3.26 has several methods to model flows with rotating components. The model under study contains two stationary sections and one rotating section. This problem can be managed by using the mixing plane approach, the multiple reference frame approach, or with a sliding mesh model. However, because the interactions between the rotor blades and stator vanes are expected to be strong, the multiple reference frame approach is not recommended (Ansys (2006a)). Therefore, only the mixing plane approach and the sliding mesh model will be considered.

The conservation equations are slightly different in a rotating domain, compared to those in a stationary domain. Figure 6.5 shows a schematic diagram of a rotating frame with respect to a stationary frame. If the rotating frame is rotating around the rotation axis with an angular velocity $\omega_{i}$, then the relative velocity $u_{r e l, i}$ (the velocity with respect to the rotational frame) is given by

$$
u_{r e l, i}=u_{i}-u_{s p i n, i}
$$

where $u_{i}$ is the absolute velocity (the velocity with respect to the stationary frame) and $u_{\text {spin }, i}=\epsilon_{i j k} \omega_{j} r_{k}$ is the spinning velocity (the velocity due to the motion of the rotating frame; $\epsilon_{i j k}$ is the Levi-Civita tensor, which is 0 if any two indices are the same, +1 if $i, j, k$ have an even permutation of $1,2,3$, or -1 if $i, j, k$ have an odd permutation of $1,2,3$.

If the density based solver is used for the simulations, FLUENT 6.3.26 modifies the momentum equations to use the absolute velocity, rather than the relative velocity. If the pressure based solver is used, then the user has a choice between the absolute velocity and relative velocity formulations of the momentum equations. 


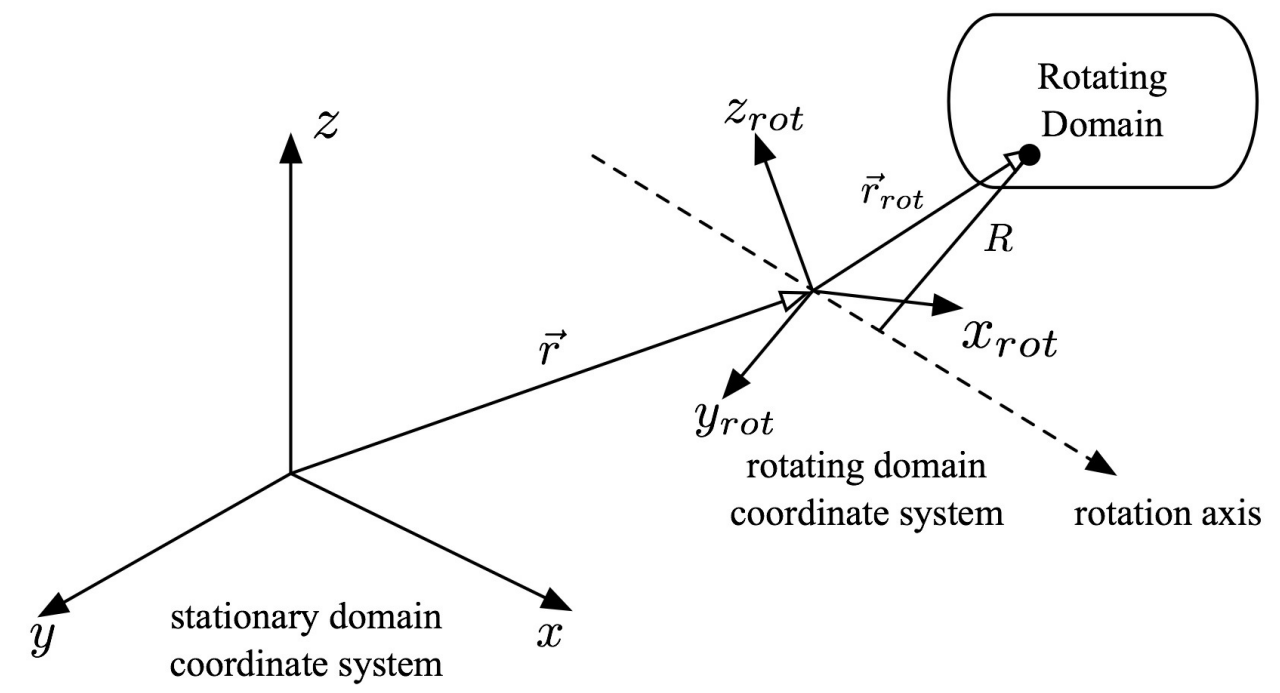

Figure 6.5: Schematic diagram of a rotating domain (based on Ansys (2006a)).

\subsubsection{Mixing Plane Model}

The mixing plane model is the preferred choice for gas turbine simulations when computational resources are limited. This model does not require the interface to be identical on both sides, so only one rotor blade and one stator vane from each stage need to be modelled. This can greatly reduce the computational domain. In addition, the mixing plane model employs a steady solver, reducing the computational time required to obtain a solution. However, this also means that the mixing plane model cannot model unsteady effects, which makes it impossible to determine unsteady interaction effects, as well as unsteady blade loading and surface properties. The mixing plane model was used for some preliminary computations and to provide initial conditions for the final unsteady simulations.

The mixing plane model artificially mixes the flow at the interface regions by 
circumferentially averaging the flow variables. The flow variables passed from the upstream domain to the downstream domain are the total pressure, flow direction cosines, total temperature, and turbulence properties. The variables passed from the downstream domain to the upstream domain are static pressure and the flow direction cosines.

\subsubsection{Sliding Mesh Model}

A sliding mesh was implemented to allow the rotor domain to rotate with respect to the stationary stator domain. As a result, unsteady simulations could be run and the rotor-stator interactions could be analyzed with minimal assumptions.

Two interface planes are required to employ the sliding mesh model. The planes must be geometrically identical, within a certain tolerance. However, the meshes on each of the faces need not be identical or conformal (if two meshes are conformal, then they are identical in all aspects, including size, shape, node locations, and connectivity between nodes). FLUENT 6.3.26 is capable of dealing with non-conformal interfaces when passing information from one domain to the next. An example of a sliding mesh interface is shown in Figure 6.6. When implementing the sliding mesh model, an interior zone (a new zone with fluid cells in both domains) and periodic zones are generated. In this example, a sliding mesh interface would consist of an interior zone $(d-b-e-c)$, where the two domains overlap, and a periodic zone pair $(a-d$ and $c-f)$, where the domains do not overlap. The fluxes of flow properties are calculated using the faces that result from the intersection of domain cell faces, such as $d-b$, rather than the actual cell zone face, such as $A$ - $B$. Therefore, in Figure 6.6, the flux into cell 4 would be determined using information from cells 1 and 3. This information would be passed through faces $d-b$ and $b$-e, in the interior zone, in the 

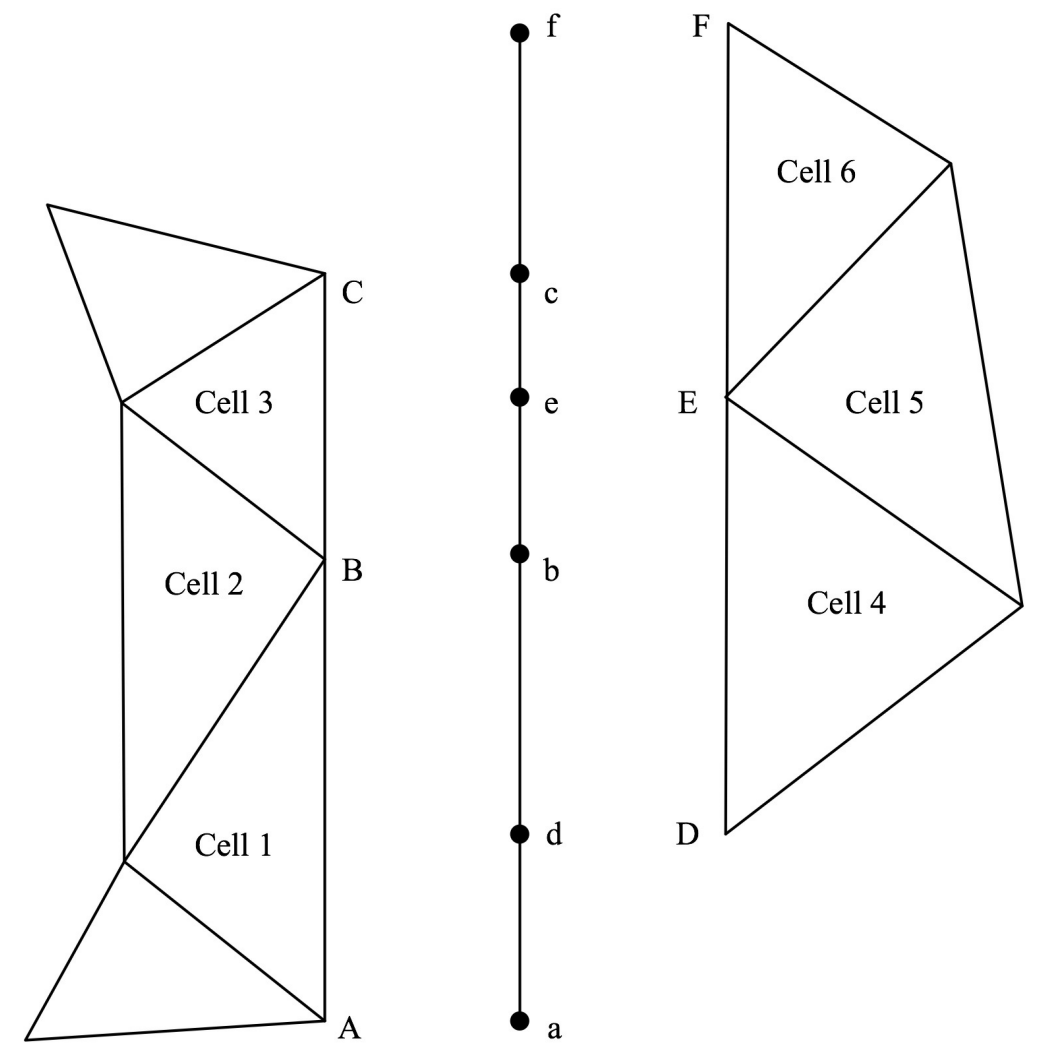

Figure 6.6: Schematic diagram of a sliding mesh interface (based on Ansys (2006a)).

same manner as if the problem were steady. Faces $a-d$ and $c-f$ are used to form a periodic pair of faces and information is transferred in a similar manner as with the interior zone.

\subsection{Simulation Methodology}

After configuring all simulation parameters and boundary conditions and initializing the solution, the reference case was initially solved by FLUENT's steady state solver. Once the steady-state configuration has sufficiently converged, it was then switched to the unsteady solver. The unsteady simulation was run with settings of increasing accuracy and 
CFL number, until the results showed sufficient convergence and periodic trends. This reference simulation was used as the initial condition for the other two cases, as the geometry and nearly all of the boundary conditions are identical.

Second order temporal and spatial discretization schemes were used, for their higher accuracy and stability. Following the procedure developed by Lastiwka (2008), a lower-accuracy turbulence model was used during the initial stages of the simulation before switching to the SST model. This provides a quick intermediate estimation for the slower, more accurate model. For the solution to converge within a small number of iterations (approximately 20) in one time step, the time step was initially set to the very small value $5 \times 10^{-6} \tau_{r}\left(\tau_{r}\right.$ is the rotor blade passing period, which is $1 / 39^{t h}$ of the rotor period). The time step was gradually increased to reduce the computational time while maintaining the solution's convergence within each time step. A time step of $2 \times 10^{-3} \tau_{r}$ (i.e. 500 time steps per blade passing period, or 1500 time steps per rotor domain pass) was found to be sufficient for resolving the desired time varying flow features. An under-relaxation factor of 0.6 for both turbulent kinetic energy and specific dissipation rate was also found to improve the stability of the simulation, most noticeably during the initial stages of simulation.

The temporal fluctuations of mass flow rate and spatially averaged static pressure were continuously monitored at the computational outlet to visually verify that the simulations reached a temporally periodic state. In addition, quantitative tests based on the discussion by Clark and Grover (2007) were performed to determine the level of periodic convergence. Figure 6.7 shows plots of convergence levels for the mean pressure, crosscorrelation factor $(\mathrm{CCF})$ at zero lag, and fractional power of the power spectrum, as well 
as the overall convergence level (OCE, i.e., the minimum value of the previous convergence levels). All examined convergence levels reached values much closer to 1 (perfect periodicity) than the convergence threshold (CT) of 0.95, as suggested by Clark and Grover (2007) to be sufficient for overall convergence.

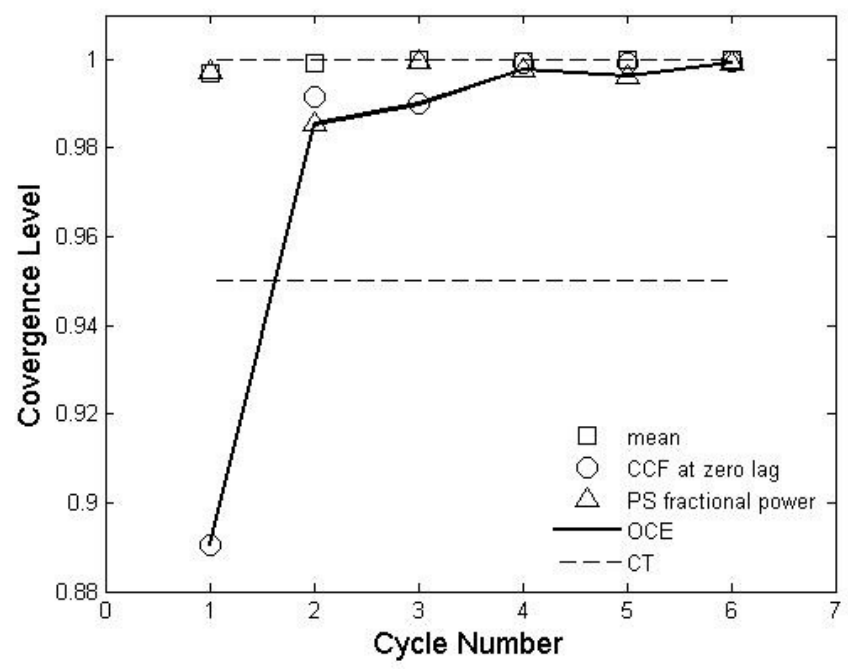

Figure 6.7: Convergence levels for reference simulation

Once the reference case had converged, the simulation files were copied and the inlet boundary conditions were set to reference the UDFs which describe the inlet total temperature fields of the VI and MP cases. These two simulations were then set to run under FLUENT's steady solver, and progressed in much the same way as the reference case, though the computation time was much reduced, as the initial conditions were much closer to the solution than those produced by FLUENT's initialization algorithms.

The procedure for the unsteady simulations was comparable to the reference, although there was a significant increase in resources required for the VI and MP cases to 
reach convergence and a periodic steady-state. Several variables were monitored for temporal periodicity, including inlet and outlet mass flow rates, mass-weighted static pressure at the rotor and duct outlets, and the drag coefficient of the stator vane. The final stages of simulation required approximately 30 to 50 iterations per time step to reach an absolute convergence level of $10^{-3}$ for continuity, velocities, and energy; the convergence levels for $k$ and $\omega$ were set to $10^{-6}$. These criteria were chosen because further reduction within the required time constraints and $10^{-3}$ is the maximum scaled residual required for a converged solution (Menter (2002)). All data used in post-processing were collected during the final cycle of simulations (one cycle period is equal to three blade passing periods, or 1500 time steps).

\subsection{Simulation Uncertainty}

In all CFD studies, the inevitable problems of uncertainty and error are strongly dependent upon the numerical code, computer architecture, and defining models. To clarify the issue, the American Institute of Aeronautics and Astronautics (AIAA) has defined uncertainty and error (AIAA (1998)) as

Uncertainty A potential deficiency in any phase or activity of the modeling process that is due to a lack of knowledge

Error A recognizable deficiency in any phase or activity of modeling and simulation that is not due to a lack of knowledge

The uncertainties and errors of any given simulation can be divided into two basic groups: acknowledged and unacknowledged. Acknowledged uncertainties and errors can 
be identified by known methods, and can possibly be reduced, eliminated, or otherwise compensated for. Unacknowledged uncertainties and errors are those which cannot be traced. The simulation uncertainties can be further broken down as (Faragher (2004))

- Acknowledged uncertainties and errors

- Physical approximation uncertainty

- Computer round-off error

- Iterative convergence error

- Discretization errors

* Spatial discretization error

* Temporal discretization errors

* Truncation errors

- Unacknowledged uncertainties and errors

- Computer programming errors

- Usage errors

Physical approximation uncertainty deals with uncertainties due to assumptions that are made during the generation of the model. Simplification to the geometry used for the simulations, turbulence modeling, simplifications to boundary conditions and neglecting heat transfer are all examples that introduce physical approximation uncertainty. An estimate of physical approximation uncertainty is possible through validation of the simulation using experimental results (Faragher (2004)). However, quantitatively validating all 
local flow parameters in an unsteady simulation, such as in turbomachinery, is very difficult due to the large volume of data. It is more appropriate to quantitatively validate global flow parameters, such as efficiency and power. Some reasons for physical approximation uncertainty are (Mehta (1996)):

- Simulation flow is not fully understood.

- Model parameters are not known to a sufficient degree of certainty.

- Models are overly simplified.

- Validation of the simulation is not possible due to lack of experimental data.

Computer round-off error develops as a result of a computer's inability to represent floating point numbers to an infinite number of decimal places. In the case of a computer that stores floating point numbers with 32 bits and single precision, which is typical of those used for the simulations in this study, data are calculated to an accuracy of $10^{-7}$. Round-off error is typically neglected because it is considered to be insignificant in comparison to other uncertainties.

Iterative convergence error exists due to the nature of the iterative process; a perfect solution will only be found after an infinite number of iterations. A criterion for stopping the simulation must therefore be adopted, in order to obtain results within a realistic time frame, and to ensure that the resulting solution is of sufficient quality. In FLUENT 6.3.26, the default stopping criterion is 


$$
\begin{aligned}
\frac{\Phi(g)}{\Phi_{\max }(g)} & <10^{-3} \\
\Phi(g) & =\sqrt{\frac{\sum_{\text {cells }}\left(\frac{\partial g}{\partial t}\right)^{2}}{m_{\text {cells }}}}
\end{aligned}
$$

where $\Phi(g)$ is the residual for a given flow parameter $g$. For the density based solver in FLUENT 6.3.26, the residual is an RMS average, over all cells, of the rate of change of a given flow parameter during one internal iteration (the iterative process during one time step). The stopping criterion is then determined by scaling the residual by the maximum residual in the first 5 iterations $\Phi_{\max }(g)$. The FLUENT 6.3.26 default scaled residual is $10^{-3}$, and the choice for this study was discussed in Section 6.5.

Discretization errors result from applying the governing equations on discrete spatial and temporal points, and are often considered the most significant errors in CFD simulations. Spatial discretization error exists because the numerical domain is meshed with a finite number of cells, whereas temporal discretization error similarly results from the application time steps which specify the time between instantaneous solutions in unsteady simulations. Spatial and temporal discretization errors can be reduced by increasing the number of grid points or by reducing the time step size, respectively. As the spatial and temporal discretizations are refined, the solution becomes progressively less sensitive. Once the sensitivity reaches 0 , the discretizations are said to be converged. If both the spatial and temporal discretizations were refined such that the respective intervals were infinitely small, then the resulting solution should, theoretically, have no discretization error. However, this is not practically possible due to the complexity of the governing equations and 
computational resource limitations. Therefore, an important aspect of any simulation is an appropriate grid convergence study to determine extent of discretization error.

A more indirect aspect of discretization error is truncation error. Truncation error is the difference between the exact non-discretized governing equations, and the finite or discretized governing equations, which are numerically solvable. The truncation error is affected by the quality of the grid in relation to localized flow features (such as shocks and boundary layers), and the order of the method used to discretize the governing equations. For example, if the lowest order term that is truncated is of first order (namely, contains $\Delta x$ as in Equation 6.7), the method is first-order accurate. Similarly, if the lowest order truncated term is of second order (contains $\Delta x^{2}$ as in Equation 6.8), the method is secondorder accurate (Faragher (2004)).

$$
\begin{aligned}
& \frac{d g}{d x}=\frac{g_{i+1}-g_{i}}{\Delta x}+\underbrace{\frac{d^{2} g}{d x^{2}} \frac{\Delta x}{2}-\frac{d^{3} g}{d x^{3}} \frac{\Delta x^{2}}{6}+\text { higher order terms }}_{\text {truncated terms }} \\
& \frac{d g}{d x}=\frac{g_{i+1}-g_{i-1}}{2 \Delta x}-\underbrace{\frac{d^{3} g}{d x^{3}} \frac{\Delta x^{2}}{6}+\text { higher order terms }}_{\text {truncated terms }}
\end{aligned}
$$

With first-order accurate methods, the leading truncated term is an even order derivative and the resulting solution will be affected by numerical dissipation or artificial viscosity (Faragher (2004)). Numerical dissipation acts to artificially increase the fluid's viscosity and smoothen out gradients. Although numerical dissipation results in inaccuracies, the fact that it also tends to stabilize the solution can be a positive attribute. Second-order accurate methods have a truncation error beginning with an odd-order derivative, meaning that the solution will show effects of numerical dispersion, which can generate oscillations 
in the solution. A combination of numerical dispersion and numerical dissipation is commonly referred to as numerical diffusion (Faragher (2004)). The final results of this study were found using simulations with second-order discretization methods in both the spatial and temporal domains to achieve high accuracy. However, first-order methods were used in the initial stages of the simulation to take advantage of their stabilizing characteristics and lower required resources.

Computer programming errors have not been studied intensively, as they are hard to quantify. These are errors that result from mistakes made while writing the code for a program used by the simulation. These errors can therefore only be addressed by the programmer. In an established commercial code, such as FLUENT 6.3.26, these errors are assumed to be negligible. Any errors remaining in the current user-defined UDF program have also been deemed to be negligible, as this program produced inlet total temperature distributions that matched expectation.

Usage errors can arise through the improper use of a particular CFD code or modelling method. This may occur during geometry or grid generation, or during the simulation itself. For example, usage error would affect a simulated turbulent flow if a laminar model were used by mistake, or a simulation of compressible flow if density were assumed to be constant. The likelihood of such errors occurring increases with the number of options in a CFD code.

In view of the experience and knowledge of the individuals and supervisors involved in this study, the probability of usage error effect on the results has been deemed negligible. Furthermore, the purpose of this study is to compare the results of different simulations 
with different inlet conditions, all of which have been conducted using otherwise-identical settings and models. This should ensure that any deviations from the actual gas turbine operation are present at the same level in all cases, which acts favourably towards the validity of a comparative evaluation of the particular effect of interest. 


\section{Chapter 7}

\section{Reference Simulation Results}

In order to study the effects of the presence and location of hot streaks on the gas turbine, it was necessary to establish a baseline for comparison. To this end, a simulation of the turbine section was performed with a uniform temperature applied to the inlet boundary, representing the common practice in turbine simulation. This uniform temperature is equal to the mass-weighted average of the hot streak temperature fields, as described in Chapter 5. All other parameters and settings were kept the same in the final, high-accuracy stages of all simulations. These conditions and the simulation procedure are explained in detail in Chapter 6. Some steady/unsteady effects are compared as well. All results refer to final unsteady simulations, unless otherwise indicated.

Many of the results presented in this chapter have been published previously (see Chang and Tavoularis (2009b) and Chang and Tavoularis (2009a)). This material has been adapted in order to document the results of the reference case so that they can be compared with the results of the hot streak simulations. 


\subsection{Simulation Verification}

Of the several methods that were found to estimate the uncertainty in simulation results, two were considered for use in the current study: the Grid Convergence Index (GCI) method of Roache (1998) and the Approximate Error Spline (AES) method of Celik and Li (2005). The GCI method is based on the Richardson Extrapolation and estimates the relative error of the numerical solution by comparison to the estimated true value. This method provides a $90 \%$ confidence estimated error band within which the true solution lies and requires that the solutions on consecutive grids achieve monotonic convergence. The AES method is based on the GCI method and also gives a $90 \%$ confidence estimated error band, but it can be implemented in cases in which the solutions on consecutive grids follow either monotonic or oscillatory convergence. It uses an approximate error to estimate the true error, which is used to find the estimated exact value of a simulation parameter and determine the GCI.

The main advantage of the AES method is that it can be used to estimate the GCI in cases for which a simulation parameter shows non-monotonic convergence as the grid is refined. Celik and Li (2005) also carried out a comparison using flow parameters which exhibited both monotonic and oscillatory convergence behaviour. The results showed that the AES method was the best performing among four different uncertainty estimation methods. The AES method was also found advantageous when the simulation parameter exhibited oscillatory convergence and three grids were used for the simulation verification. The AES method was chosen over the basic GCI method for this study. For a more in-depth description of these two verification methods, see Lastiwka (2008). 
The AES results of the steady grid convergence study is shown in Figure 7.1, for three grids of varying cell counts $(1.2,1.7$, and 2.2 million), as described in Section 6.2.1. The dashed line indicates the estimated exact value computed using the AES method, and the error bars show the GCI for each of the grids. The GCI for each grid was determined to be $14.73 \%, 3.36 \%$, and $1.25 \%$, respectively, using the AES-estimated exact value.

Note that the GCI and AES data have been contributed by the author, and have not been published previously.

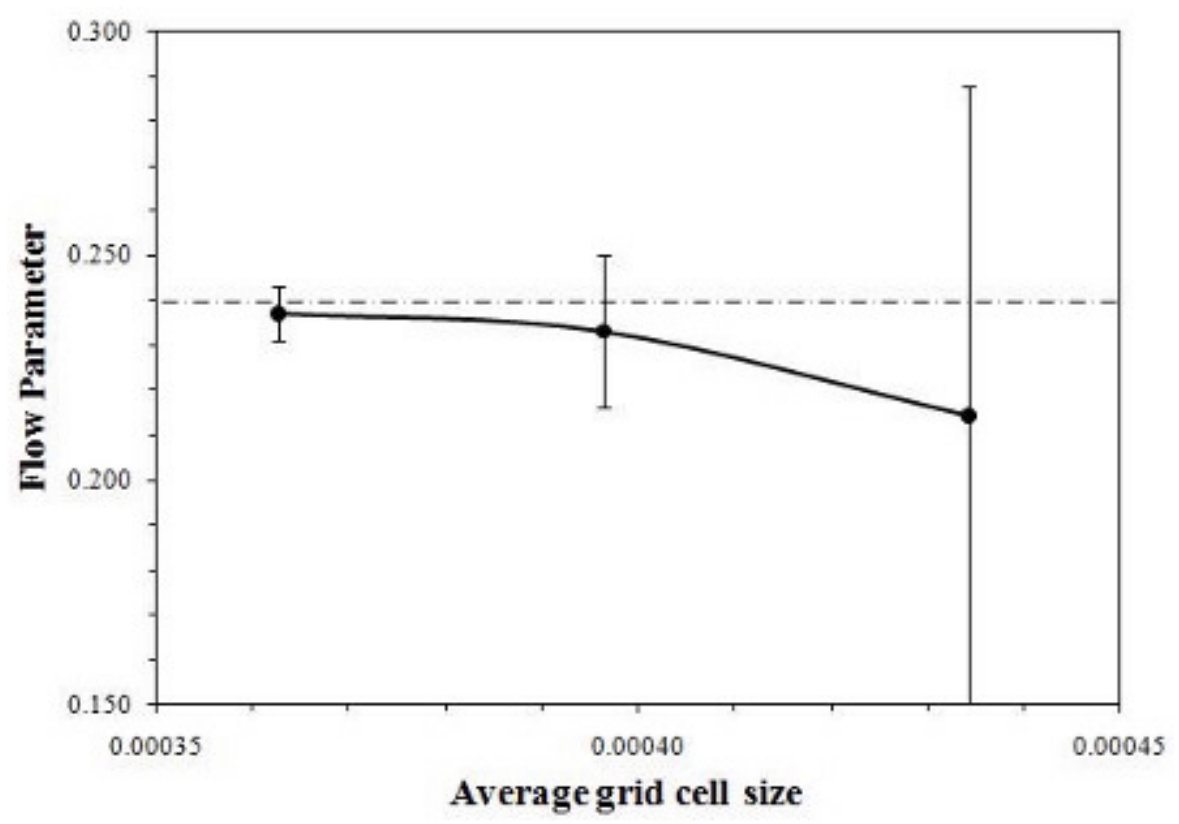

Figure 7.1: Flow parameter computed using three grids of decreasing cell size. Error bars show GCI for each grid, and dashed line indicates exact value as estimated by the AES method.

\subsubsection{Comparison Between Steady and Unsteady Simulations}

Coherent vortices identified in the rotor using RANS (Reynolds-averaged NavierStokes) and URANS (unsteady Reynolds-averaged Navier-Stokes) are presented in Fig. 7.2. 
The sizes and the locations of the rotor casing passage vortices and the rotor hub passage vortices were comparable in both simulations. However, the horseshoe vortices and the tip leakage vortices in RANS were smaller than those in URANS. The tip leakage vortices in the URANS simulation started rolling up near the mid-chord section, while the tip leakage vortices in RANS first appeared between the mid-chords and the trailing edges of blades. Finally, the axial gap vortex observed in URANS was not at all identified using RANS.

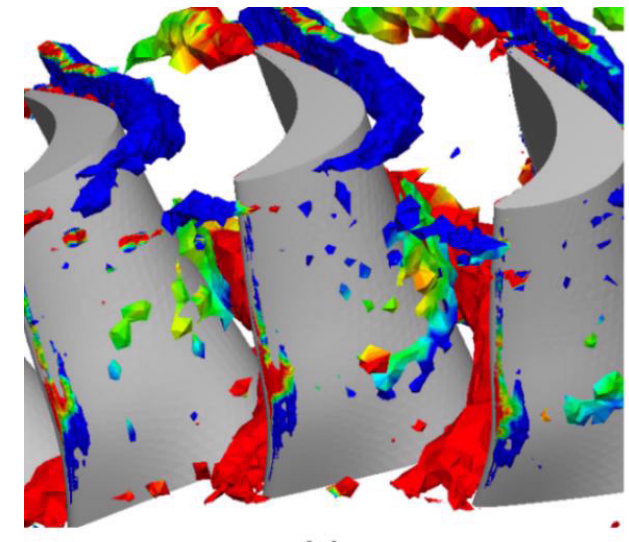

(a)

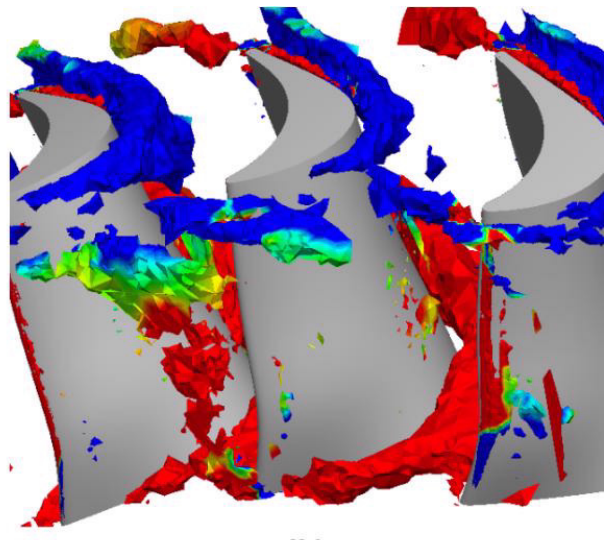

(b)

Figure 7.2: Vortices in the rotor section, coloured by axial vorticity: (a) steady contours from RANS, (b) instantaneous contours from URANS. From Chang and Tavoularis (2009a).

Contours of the time-averaged static pressure at 50\% span are depicted in Fig. 7.3 for the steady and unsteady simulations. The static pressure in the aft-midsection on the vane suction sides from RANS was lower than the corresponding time-averaged pressure from URANS. There was also a sharp increase in static pressure near the trailing edges of the vanes in RANS but not in URANS. These differences in static pressure were mainly due to the unsteadiness, which is not considered in RANS.

Figure 7.4 shows entropy distributions at $50 \%$ span for the steady and unsteady simulations. Relatively high entropy indicates high viscous losses and marks boundary 


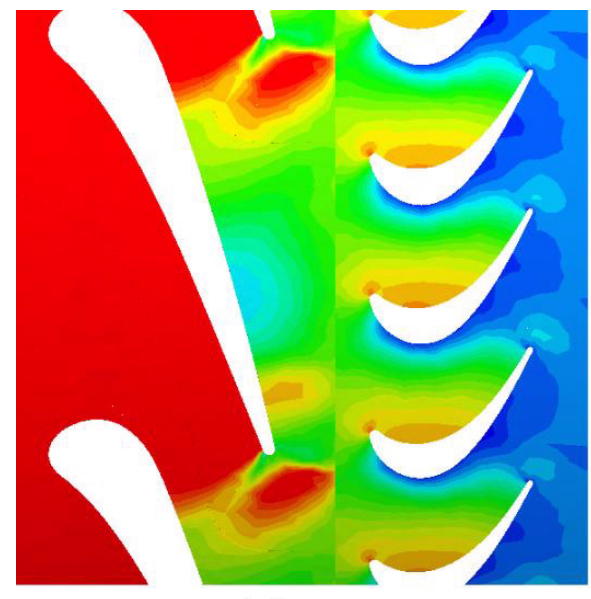

(a)

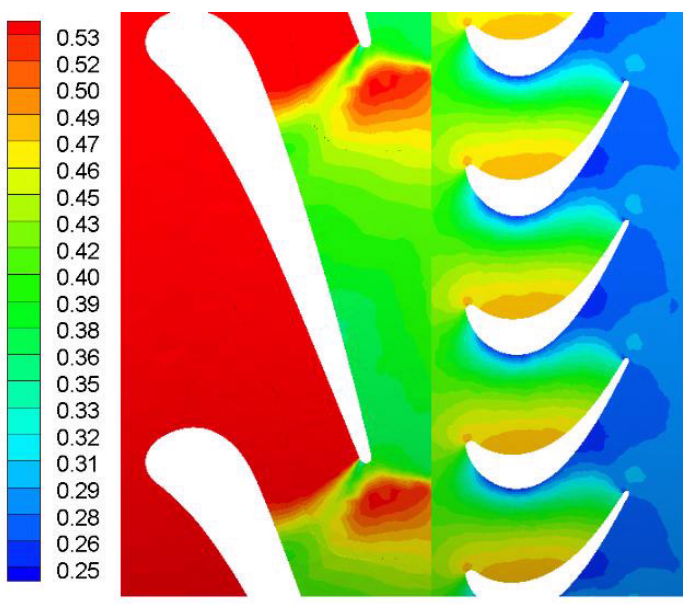

(b)

Figure 7.3: Contours of static pressure, normalized by $P_{o 1}$, at $50 \%$ span: (a) steady contours from RANS, (b) time-averaged contours from URANS. From Chang and Tavoularis (2009a).

layers, wakes, and other viscous regions in the flow. While there were very few differences in the vane wakes within the stator section for both URANS and RANS, the wakes of the blades were appreciably stronger in the URANS simulation, and the losses within the blade passages were also higher in the URANS.

Figure 7.5 shows contours of the total pressure loss coefficient in the rotor reference frame. The relative total pressure loss coefficient at the rotor outlet is defined as

$$
Y_{r}=\frac{\widehat{P}_{o 2 r}-P_{o 3 r}}{\widehat{P}_{o 3 r}-\widehat{P}_{3}}
$$

where the mass-weighted pressure and relative total pressure are, respectively,

$$
\widehat{P}=\frac{\int P \rho V \cdot d A}{\int \rho V \cdot d A} \quad \text { and } \quad \widehat{P}_{o r}=\frac{\int P_{o r} \rho V \cdot d A}{\int \rho V \cdot d A}
$$

The total pressure loss from URANS was found to be highest near the midspan of the rotor outlet, and decreased towards the endwalls. This high loss may be related to the rotor blade wakes shown in Fig. 7.4. In contrast, the total pressure losses from RANS 


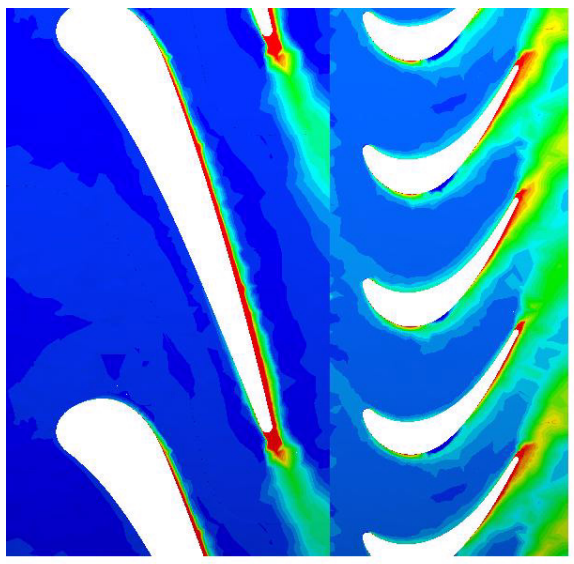

(a)

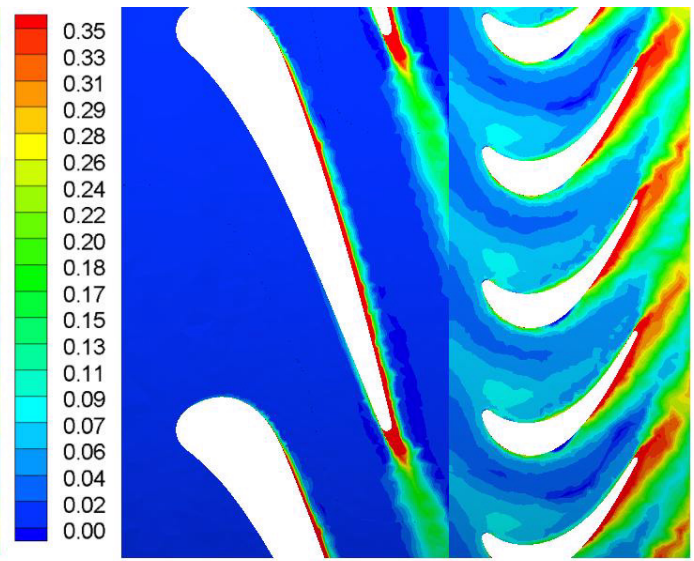

(b)

Figure 7.4: Contours of entropy, normalized by $\mathbf{R}$, at 50\% span: (a) steady contours from RANS, (b) time-averaged contours from URANS. From Chang and Tavoularis (2009a).

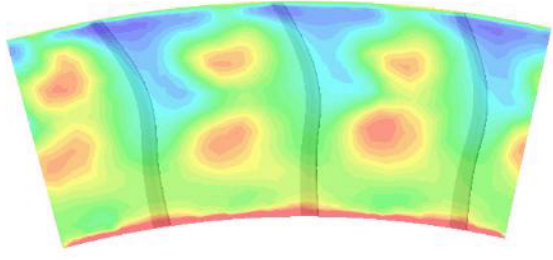

(a)
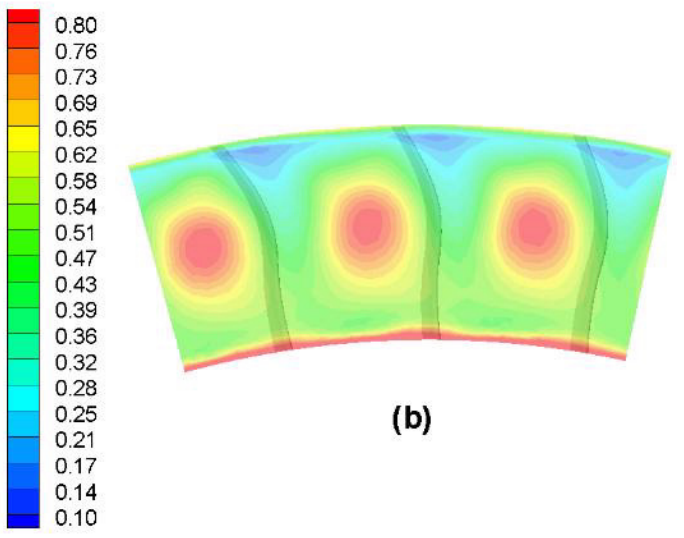

(b)

Figure 7.5: Contours of relative total pressure loss coefficient at the rotor outlet plane: (a) steady contours from RANS, (b) time-averaged contours from URANS. View is from downstream of the rotor outlet plane. From Chang and Tavoularis (2009a). 
showed two peaks.

The differences in mass-weighted total pressure coefficients are shown in Table 7.1, indicating that the total pressure losses from URANS were measurably higher than those from RANS. The percent difference of the relative total pressure loss coefficients at the rotor outlet was $10.9 \%$, which is comparable to the $10 \%$ value reported by Pullan (2006) for rotor-only simulations.

\begin{tabular}{|l|c|c|}
\cline { 2 - 3 } \multicolumn{1}{c|}{} & $\begin{array}{c}\text { Total pressure loss }(Y) \\
\text { at stator outlet }\end{array}$ & $\begin{array}{c}\text { Relative total pressure loss }\left(Y_{r}\right) \\
\text { at rotor outlet }\end{array}$ \\
\hline \hline RANS & 0.6053 & 0.4477 \\
\hline URANS & 0.6257 & 0.5025 \\
\hline Difference (\%) & 3.3 & 10.9 \\
\hline
\end{tabular}

Table 7.1: Comparison of mass-weighted total pressure loss coefficients.

Figure 7.6 shows the static pressure distributions on the blade suction side, as determined using RANS and URANS. The URANS results indicated the formation of a local low-pressure region near the mid-span toward the leading edge of blades, which was isolated from the low-pressure regions formed by the two passage vortices. In the RANS results, the three low-pressure regions were more strongly connected. It was reported by Chang and Tavoularis (2009b) that the local low-pressure regions were highly correlated to the locations of blade passage vortices, tip leakage vortices, and crown vortices. Another visible difference is that the pressure near the forward part of the blade tips was stronger in the URANS simulation than under RANS.

In addition to the observed differences in time-averaged properties, unsteady simulations have the obvious advantage of resolving the temporal fluctuations of the pressure, velocity, and other properties, which can then be used in vibratory stress analyses, an es- 


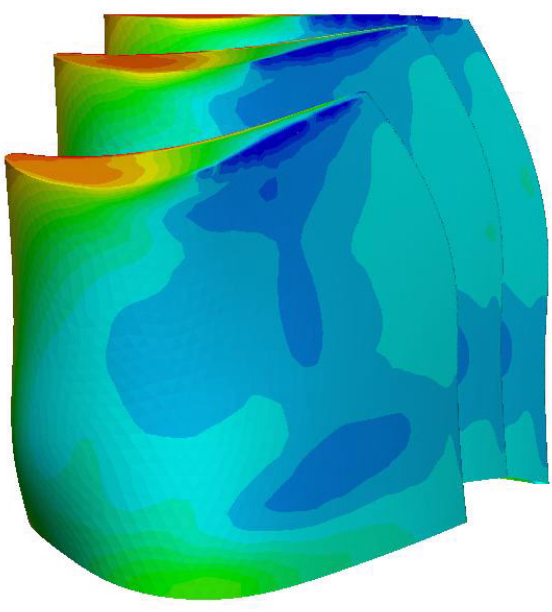

(a)

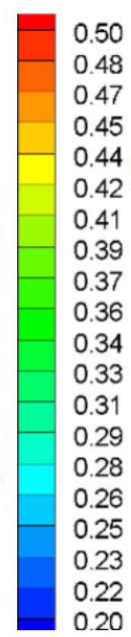

0

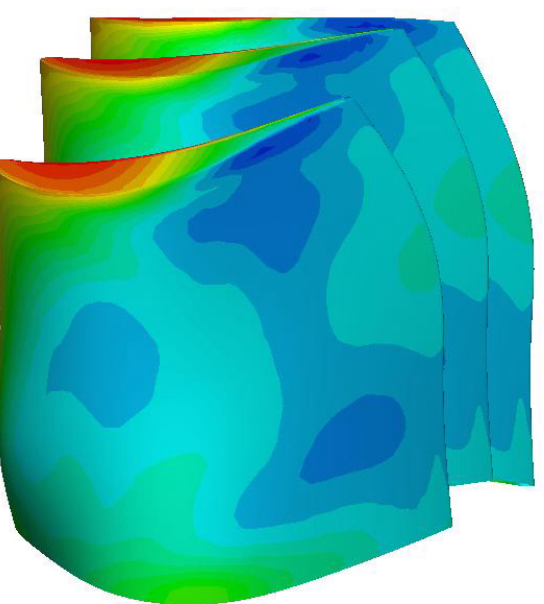

(b)

Figure 7.6: Contours of static pressure normalized by total inlet pressure: (a) steady contours from RANS, (b) time-averaged contours from URANS. From Chang and Tavoularis (2009a).

sential aspect of high-pressure turbine design. Consequently, the periodically-forming axial gap vortices and crown vortices were identified in the unsteady simulations, but could not be detected in the steady ones.

\subsection{Coherent Vortices}

Coherent structures reported in previous literature include casing and hub horseshoe vortices in the stator and rotor sections, tip leakage vortices in the rotor section, and vortices shed by the trailing edges of vanes and blades (Payne (2001)). Vortices associated with the stator section are quasi-stationary, whereas vortices related to the rotor section are quasi-stationary in the rotating frame of reference. Note that, in the present context, the term stationary is used to indicate that the position of the vortex is fixed in space and not that the vortex characteristics are statistically independent of time origin, as stationarity 
signifies in turbulence theory.

For the most part, the vortical coherent structures showed very little differentiation between the reference simulation and those using non-uniform hot streak inlet conditions. Unless otherwise noted, the configuration and characteristics of the coherent structures were essentially identical for the three inlet configurations of interest.

\subsubsection{Coherent Structure Identification}

Due to the highly complex and turbulent flow that exists in turbomachines, it is quite difficult to distinguish coherent structures (CS) from non-coherent turbulence and mean vorticity patterns (Chang and Tavoularis (2007)). Although there is no currently accepted universal method for identifying coherent vortical structures, most of the popular criteria are based on pointwise kinematic analysis of the velocity gradient tensor, thereby making them Galilean invariant (Chakraborty et al. (2005)). Pointwise identification methods define a function to be evaluated point-by-point in order to classify each point as part of or outside a vortex, based on certain criteria. The complex, turbulent nature of turbomachine flow can prove a difficult environment in which to distinguish coherent structures from non-coherent turbulence and mean vorticity patterns (Chang and Tavoularis (2007)). For this reason, it is important to use an identification method with the capability to discern between the large-scale structures of interest and the extraneous turbulent fluid.

The $\lambda_{2}$ criterion (Jeong and Hussain (1995)) is based on the observation that the concept of a local pressure minimum in a plane fails to identify vortices under strong unsteady and viscous effects. By neglecting these effects, the symmetric part of the incom- 
pressible Navier-Stokes equation can be expressed as

$$
S^{2}+\Omega^{2}=-\frac{1}{\rho} \nabla(\nabla P)
$$

where $P$ is the pressure and the equation is a representation of the pressure Hessian. To capture the region of local pressure minimum in a plane, Jeong and Hussain (1995) define the vortex core as a connected region with two positive eigenvalues of the pressure Hessian. If the eigenvalues of the symmetric tensor $S^{2}+\Omega^{2}$ are ordered as $\lambda_{1} \geq \lambda_{2} \geq \lambda_{3}$, this definition is equivalent to the requirement that $\lambda_{2}<0$ at every point inside the vortex core. It has been shown that, while the $\lambda_{2}$ criterion measures the excess of rotation rate over the strain rate magnitude only on a specific plane, the following $Q$ criterion looks for this excess in all directions.

The $Q$ criterion (Hunt et al. (1988)) identifies vortices as flow regions with positive second invariant of $\nabla u$. This method allows for the capture of large-scale vortical structures whose core fluid has small, if any, strain. Positive values of $Q$ indicate regions where the vorticity overcomes strain. Although intended for use in incompressible analysis, this identification method remains valid for many compressible flows (Dubief and Delcayre (2000)). The advantage of this method is that the value of $Q$ is not affected by wall-generated shear because the latter has equal components of rotation and strain rate. CS have been identified as surfaces on which $Q$ is maintained above a particular value, called the threshold (Chang and Tavoularis (2007)). However, in some flows, the original $Q$ criterion is sensitive to the choice of threshold.

An adaptation of the $Q$ criterion was used for this study. The use of the basic method to analyze the results of the present simulation identified a large number of CS in 
the near-wall regions but did not clearly identify the tip-gap vortices or the passage vortices. To resolve this issue, the parameter $Q$ was first modified by Chang and Tavoularis (2007), such that

$$
Q_{m}=\frac{1}{2}\left(\Omega_{i j} \Omega_{i j}-c_{q} S_{i j} S_{i j}\right)
$$

where $c_{q}$ is an empirical factor near 1 that reduces the effect of the rate of strain on the coherent structure identification. Using values of $c_{q} \neq 1$ increases or decreases the effect of strain rate on the coherent structure identification process, which can help to identify surfaces of realistic coherent structures with less sensitivity to threshold choice. By selecting $c_{q}=1.25$ and $Q=1 s^{-2}$, the tip-gap vortices and the passage vortices were identified, while the small-scale, near-wall CS were filtered out.

Another method of identifying coherent vortical structures has been developed by Zhang and Choudhury (2006), which is based on the widely-used helicity density $(H)$ scheme. Their eigen helicity density $\left(H_{e}\right)$ scheme is Galilean invariant (unlike the original $H$ scheme) and attempts to incorporate advantages of existing methods by considering the full $\nabla u$ eigen system. Zhang et al. (2007) demonstrate the suitability of their scheme for use in turbomachinery analyses, which seems to improve upon the previous popular schemes. However, this scheme is not readily implemented in the current version of Fluent, making it unsuitable for use in this study. 


\subsubsection{Stationary Vortices}

Several large-scale vortices are known to form in high-pressure turbine stages and significantly contribute to the flow field and therefore the time dependent loading of the vanes and blades (Payne (2001)). The present simulations have successfully identified the formation of various types of quasi-stationary vortices by employing the modified $Q$ criterion (Fig. 7.7). Most of the previously-listed structures have been identified, but not the stator hub horseshoe and passage vortices. These vortices are dependent on the upstream duct geometry and it is possible that, in this computational geometry, hub vortices in the stator were relatively weak. Emphasis was not placed on the identification of these vortices, as this study is more focused on effects in the more sensitive rotor section.

The rotor passage vortices started to develop near the leading edges of the rotor blades and grew in size while moving downstream. In addition, the axis of each passage vortex shifted from the rotor hub and casing endwalls toward the mid-span region of the blades. This is illustrated by vortices 5 and 7 in Fig. $7.7 \mathrm{~d}$ as they moved from left to right. The tip leakage vortices (number 8 in Fig. 7.7) were closer to the suction sides of the rotor blades than any other vortices (Lakshminarayana (1996)). They began rolling up near the mid-chord region and were continuously visible until they reached the trailing edge. Their identification was then intermittent over a short distance, and were identified again just downstream of the blade trailing edges.

The modified Q criterion clearly identified the locations and sizes of the observed vortices, but did not discern direction of rotation. The rotational directions of vortices were identified in Fig. 7.7b-d by colouring the vortex surfaces according to the local value of 

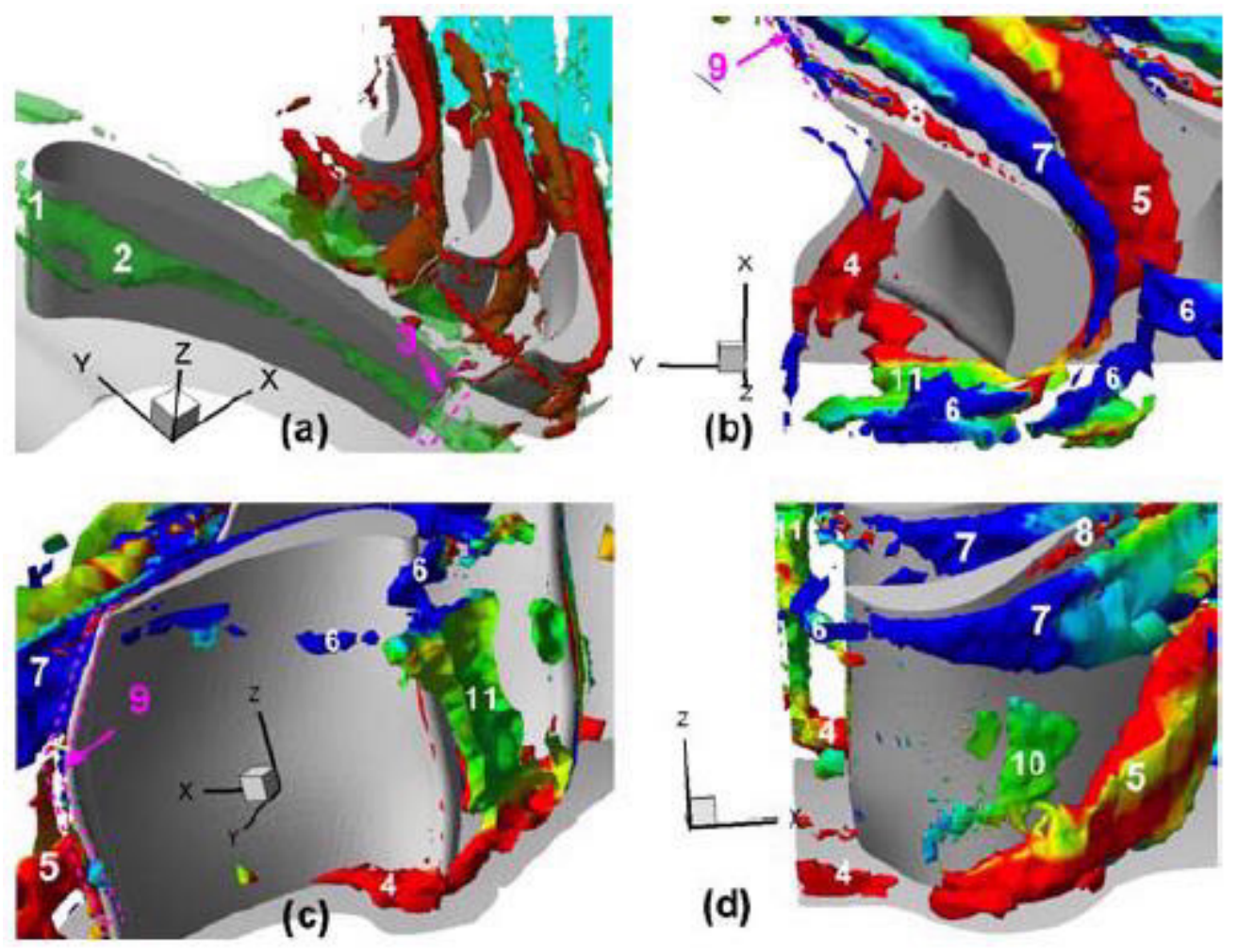

Figure 7.7: Vortices identified by the modified Q criterion: (a) vortices in the stator (green), the rotor (red) and the extended duct (light blue); (b) top view of vortices in the rotor coloured by axial vorticity value; (c) left view of vortices in the rotor; (d) right view of vortices in the rotor. Vortex types: stator casing horseshoe (1), stator casing passage (2), stator trailing edge (3), rotor hub horseshoe (4), rotor hub passage (5), rotor casing horseshoe (6), rotor casing passage (7), rotor tip leakage (8), rotor trailing edge (9), rotor crown (10), axial gap (11). From Chang and Tavoularis (2009b). 
the axial component of vorticity. Within the rotor passage, streamwise vortices tended to move downstream as counter-rotating pairs. For example, shortly after the formation of the tip-leakage vortex, it was drawn closer to the counter-rotating casing passage vortex. Furthermore, the casing passage vortex and the hub passage vortex were initially quite distant, then grew in size and approach each other as they passed over the surface of the rotor blade.

The effects of the vortices over the rotor blade can be seen by comparing Fig. 7.8a to the rest of Fig. 7.8. Figure 7.8b shows pathlines as they flowed over the surface of the rotor blade, after being released from different surface locations. The paths show that the casing passage vortex induced near-wall fluid to move away from the casing, while the hub passage vortex caused near-wall fluid to move away from the hub. Both fluid paths reached near the midspan region of the blade by the time they approached the trailing edge. Three separation lines are easily identified, and are indicated by dashed black lines: the uppermost between the casing passage vortex and the tip leakage vortex; a second between the casing passage vortex and the core flow over the suction surface; and a third between the suction core flow and the hub passage vortex (the latter was also reported by Miller et al. (2003)). Contours of surface pressure and wall shear stress are shown in Fig. 7.8c and Fig. 7.8d, respectively. The contour patterns illustrate that the presence of all three vortices on the suction surface of the blade were indicated by local regions of low pressure and high wall shear stress.

Figure 7.9 shows cross-sections of vortices as identified at two different times downstream of the rotor blade trailing edges. The contours are of the modified $Q$ criterion on 


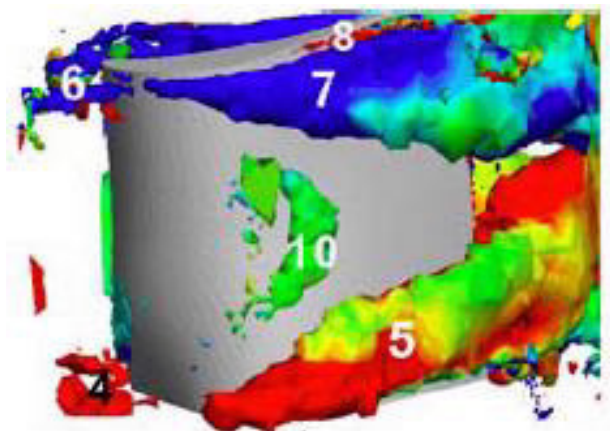

(a)

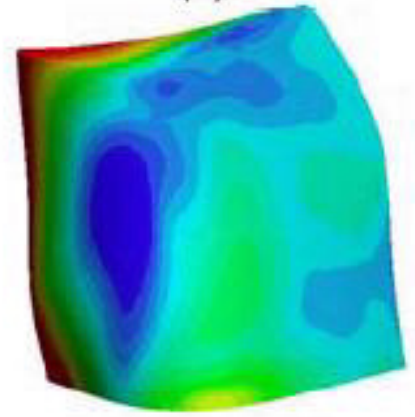

(c)

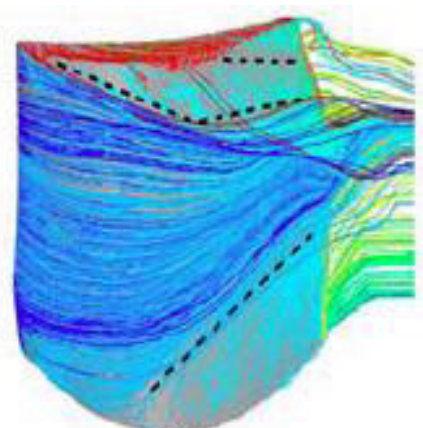

(b)

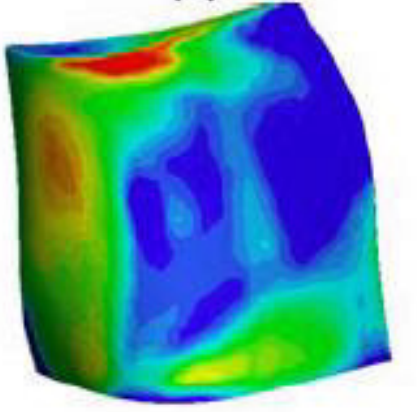

(d)

Figure 7.8: Effects of vortices on the suction side of rotor blade at $t / \tau_{r}=0.5$ : (a) vortices coloured by axial vorticity (red for counterclockwise rotation, blue for clockwise); (b) pathlines released from the blade surface (red lines from the blade tip, dark blue from the leading edge, light blue from the suction side, and yellow from the trailing edge of the blade; dashed black lines mark separation lines); (c) surface pressure isocontours, normalized by the total inlet pressure (d) wall shear stress. From Chang and Tavoularis (2009b). 
a plane which is normal to the turbine axis, and is downstream of the rotor blade trailing edges by a distance of $10 \%$ of the axial blade chord. The two passage vortices and the tip leakage vortex were quasi-stationary with respect to the rotor reference frame, although their shapes and sizes varied somewhat with the rotor's movement. The distance between the casing passage vortex and the hub passage vortex downstream of the indicated blade $M$ was reduced as time passed from $t / \tau_{r}=0.0$ to $t / \tau_{r}=0.5$. This resulted from the combined effects of size increase of both passage vortices and a spanwise shift of the hub passage vortex. One may also observe that three different portions of the hub passage vortex may be identified behind blade $\mathrm{R}$ at $t / \tau_{r}=0.5$. This can be attributed to the vane trailing edge shock, to be discussed in Section 7.3.1.

\subsubsection{Axial Gap Vortices}

In addition to the well known quasi-stationary vortices mentioned previously, the reference simulation identified the formation and disappearance of unsteady vortices located in the axial gap between the stator and rotor sections. The axes of these axial gap vortices were roughly parallel to the stator trailing edge and the rotor leading edge, as seen in Fig. 7.10.

Observation of the motion of these vortices, showed that they were nearly stationary in space with respect to the vane reference frame, but strongly time dependent, as they appeared and dissipated with a period equal to that of a the passing blade $\tau_{r}$. The axial gap vortices were initially identified near the rotor hub and grew in length toward the rotor

casing as time passed, until some time at which they ceased to be identified by the modified $Q$ criterion. The rotation direction of an axial gap vortex was such that, when viewed along 

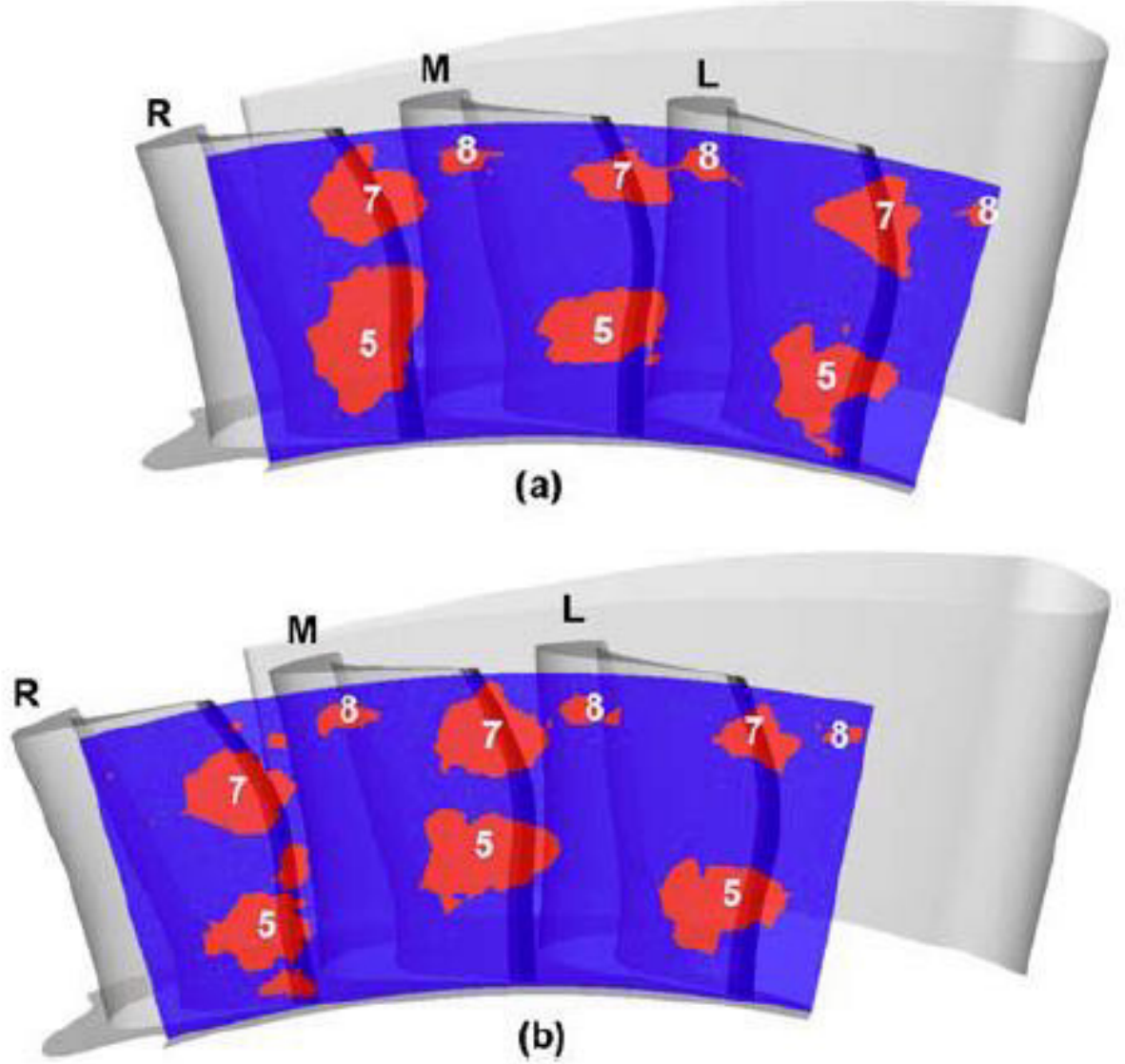

Figure 7.9: Modified Q contours 10\% of $c_{r}$ (blade axial chord) downstream of rotor trailing edge at (a) $t / \tau_{r}=0.0$, and (b) $t / \tau_{r}=0.5$. Identified cross-sections include that of the rotor hub passage vortex (5), the rotor casing passage vortex (7), and the tip leakage vortex (8). View is from downstream of the rotor section in the upstream direction. From Chang and Tavoularis (2009b). 
its axis from the casing towards the hub, it would rotate clockwise. Thus, the vorticity of an axial gap vortex was approximately in the same direction as the vorticity on the suction side of the vane (clockwise) and in the opposite direction to the vorticity on the pressure side of the vane (counterclockwise).

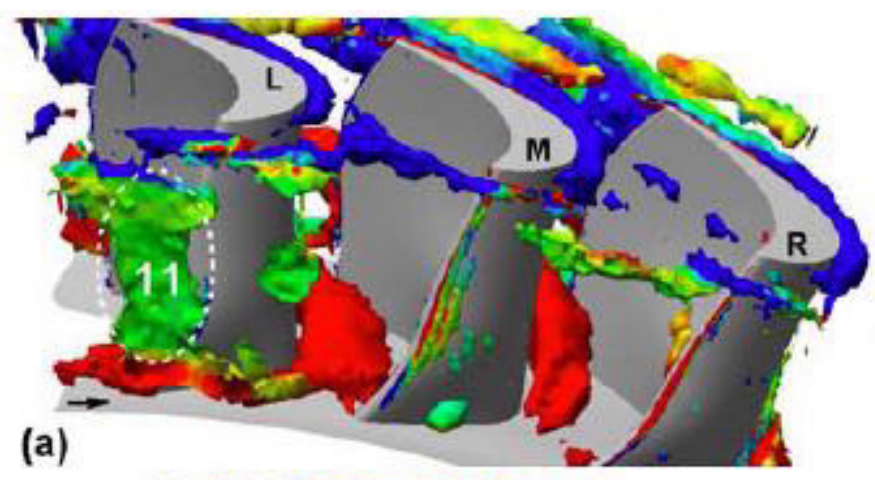

\section{(a)}

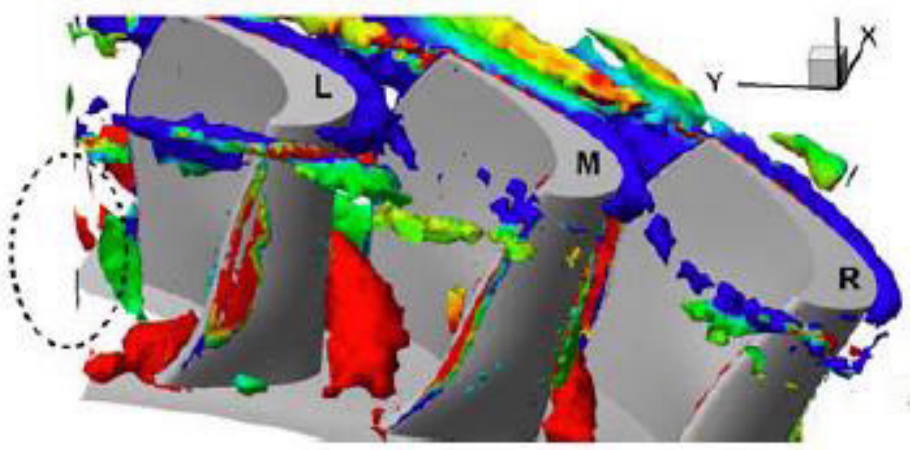

Figure 7.10: Illustration showing the location of the axial gap vortex (11), remaining axially upstream of the leading edge of the rotor blade, (a) as the vortex forms at $t / \tau_{r}=0.0$, and (b) as it disappears at $t / \tau_{r}=0.5$ (see 7.11 for the relative vane/blade positions at these times). From Chang and Tavoularis (2009b).

Based on this observation, the following scenario for the generation and disintegration of the axial gap vortex was devised:

- When there is no blade in the path of the vane wake, the wake convects downstream with both edges maintaining their respective vorticities, which are opposite in direction. 
- When a blade passes nearby such that its suction side is near the edge of the wake, it strengthens the clockwise vorticity on one side of the vane wake to the point that it develops a standing axial gap vortex.

- When the blade's pressure side passes by, with its clockwise vorticity, the vortex dissipates.

To help visualize the effect of an axial gap vortex on the unsteady flow pattern past the blade, Fig. 7.11 shows instantaneous contours of specific entropy, which clearly mark the location of the vane wake (Miller et al. (2003)), at two representative times during one blade passing period.
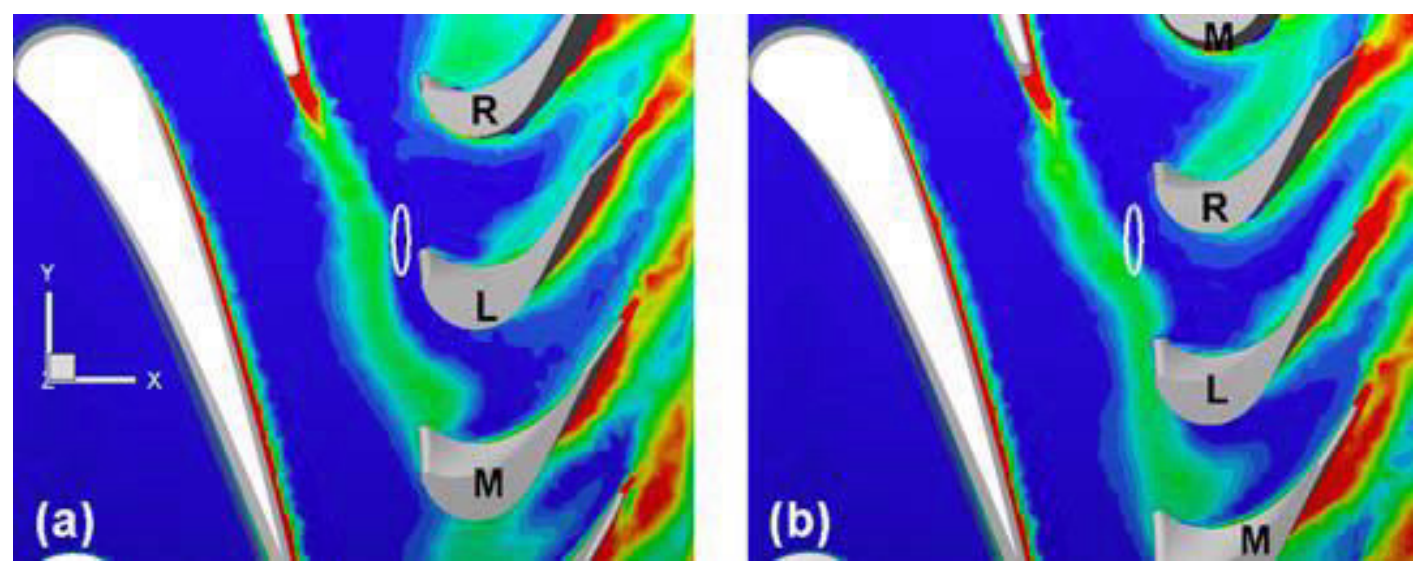

Figure 7.11: Instantaneous entropy contours at $50 \%$ blade span; a white ellipse marks the location of the axial gap vortex; (a) $t / \tau_{r}=0.0$ (strong vortex), (b) $t / \tau_{r}=0.5$ (no vortex identified). From Chang and Tavoularis (2009b).

A strong counterclockwise axial gap vortex was present upstream of the rotor (Fig. 7.11a), when the wake faced the suction side of blade $L$. No axial gap vortex was present after blade $L$ moved away (Fig. 7.11b), and the vane wake impinged on the leading edge of blade $L$. 
Figure 7.12 shows instantaneous contours of static pressure, normalized by the total inlet pressure, at $50 \%$ of the blade span. The range of contour levels was intentionally truncated, to enable one to see details in the axial gap region between the rotor and the stator. The vane trailing edge shock location was identified by inspection and indicated for clarity by a dashed line.

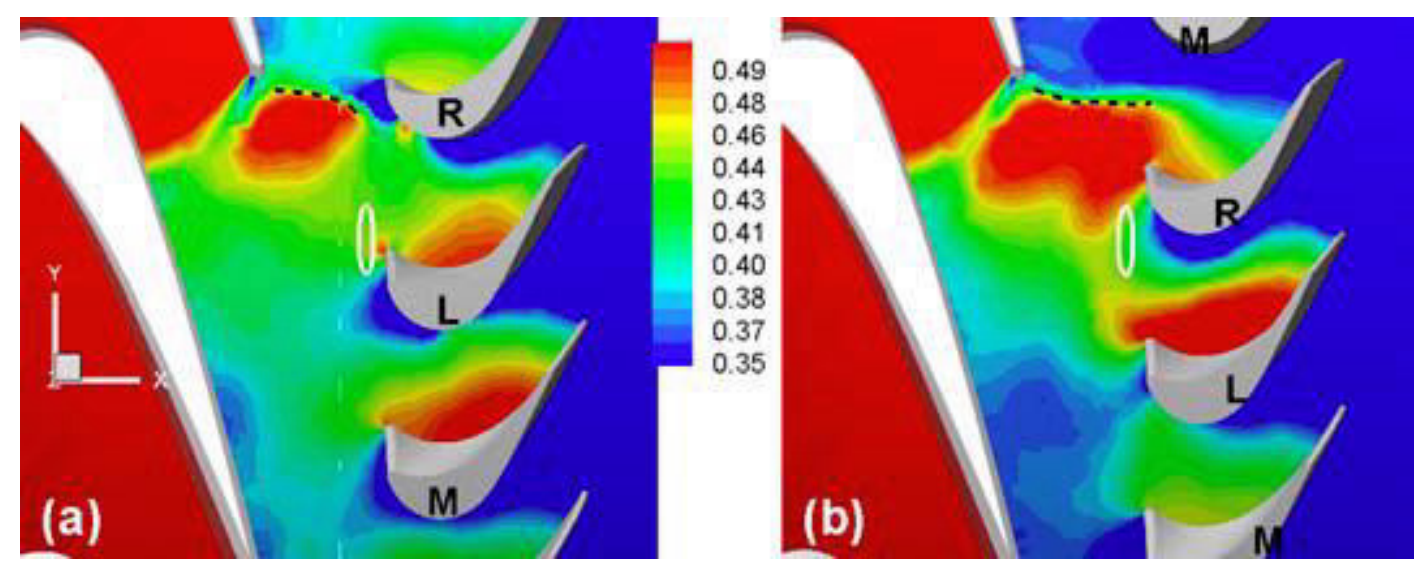

Figure 7.12: Contours of instantaneous static pressure, normalized by the total inlet pressure, at $50 \%$ blade span; a white ellipse marks the location of the axial gap vortex, whereas a hand-drawn dashed black line indicates a possible shock location; (a) $t / \tau_{r}=0.0$ (strong vortex), (b) $t / \tau_{r}=0.5$ (no vortex identified). From Chang and Tavoularis (2009b).

A change in the average shock angle is quite noticeable from $t / \tau_{r}=0.0$, when the shock impinged on the suction surface of blade $R$ (Fig. 7.12a), to $t / \tau_{r}=0.5$, when the shock passed between blades $M$ and $R$ (Fig. 7.12b). At $t / \tau_{r}=0.0$, the axial gap vortex, indicated by a hand-drawn ellipse, was quite strong and just upstream of the stagnation region of blade $L$.

The axial gap vortex was bounded by the low pressure region near the suction side of blade $L$ and the high pressure region near the leading edge of the same blade (Fig. 7.12a). As shown in Fig. 7.12b, a strong favourable pressure gradient was present near the 
forward part of the suction side of blade $R$. This promoted the following of the local flow direction along the curvature of the suction side of blade $R$, which seems to be associated with the disappearance of the axial gap vortex.

\subsubsection{Crown Vortices}

A second type of vortex, as yet undocumented in literature, is apparent in Fig. 7.7d and, even more clearly, in Fig. 7.8a. The axis of this vortex was aligned approximately to the turbine's radial direction, and was located between the casing passage vortex and the hub passage vortex near the crown of the rotor blade. In the absence of any previous nomenclature, this vortex will be called the crown vortex. The formation process of this vortex is not yet entirely clear. It appeared to form when the vane wake migrated from the pressure side of the rotor blade toward the crown on the suction side of the next oncoming blade due to the pitchwise pressure gradient (see the wake passage between the blade $R$ and $M$ in Fig. 7.11b). Therefore, the crown vortex possibly formed as a result of strong shearing between the fluid of high loss and low momentum in the stator wake and the high momentum fluid in the core of the blade passage.

\subsection{Flow Field Characteristics}

\subsubsection{Shock Waves and Potential Fields}

Figure 7.13 shows contours of blade surface pressure as well as in-flow static pressure contours on planes at $50 \%$ and $10 \%$ of the blade span at two different times. The approximate locations of shock waves originating on the trailing edge of the upstream vane 
are marked by dashed lines. At $t / \tau_{r}=0.0$, the shock impinged on the suction side of blade $\mathrm{R}$ along the entire span of the blade, but, as time passed, blade $R$ moved past the upstream vane trailing edge, moving the shock toward its leading edge.
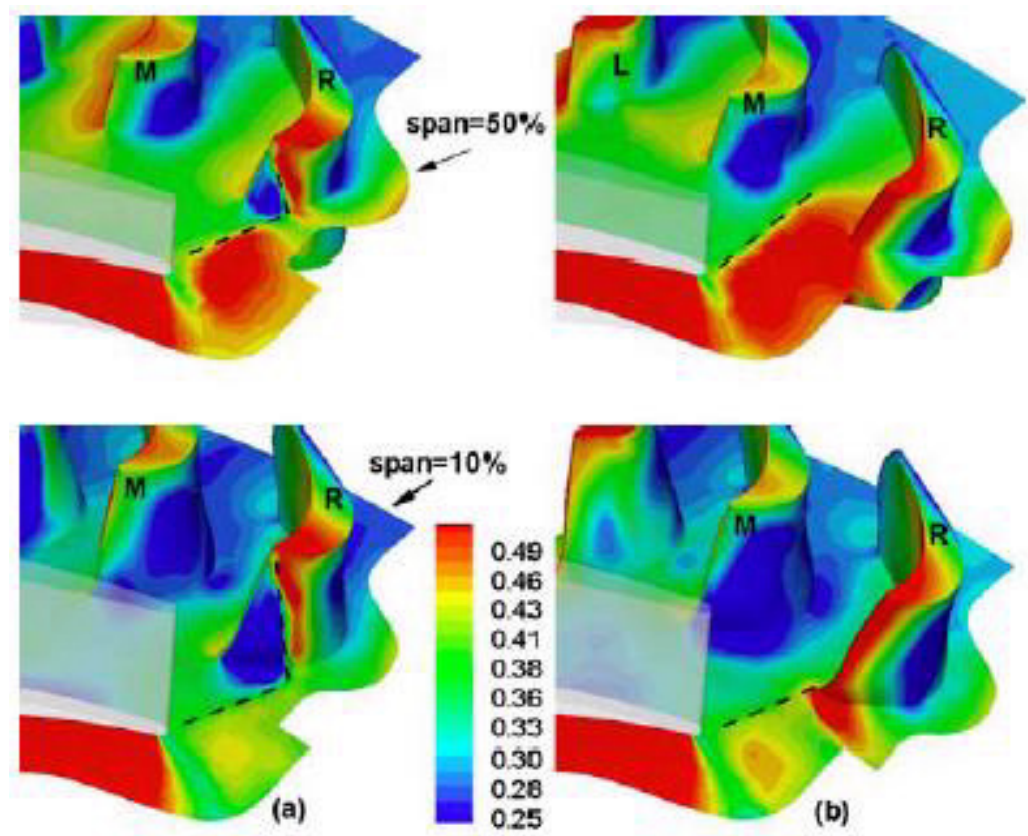

Figure 7.13: Instantaneous contours of static pressure normalized by the total inlet pressure on blade surfaces and on two planes in the flow, at $50 \%$ and $10 \%$ span; (a) $t / \tau_{r}=0.0$, (b) $t / \tau_{r}=0.5$; dashed lines indicate possible vane shock locations. From Chang and Tavoularis (2009b).

Strong temporal fluctuations of surface pressure have been observed on blade suction sides, near the leading edge. These fluctuations contributed considerably to the unsteady loading of the blade and were mainly attributed to the unsteady shock impingement (Miller et al. (2003)). Figure 7.13 also illustrates the strong spanwise variation of the impact of the vane shock on the blade surface, in both strength and relative position.

The variation of local static pressure in the downstream half of the blade passage is shown more clearly in Fig. 7.14, in which the range of contours has been truncated to 
appropriate values.

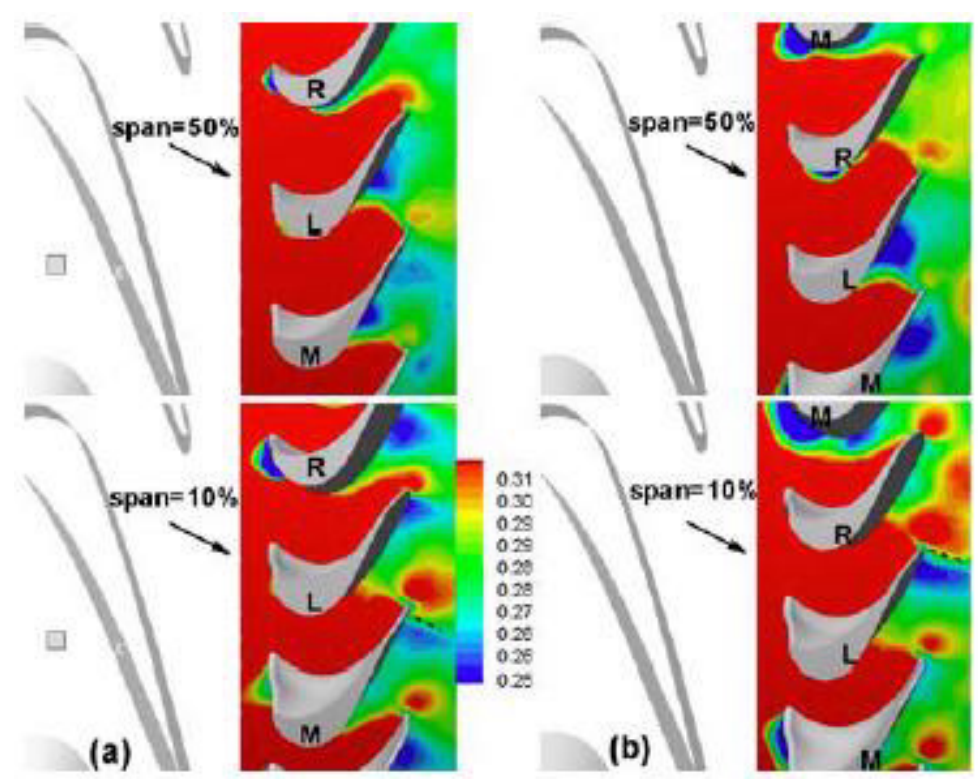

Figure 7.14: Instantaneous contours of static pressure normalized by the total inlet pressure on two planes in the flow, at $50 \%$ and $10 \% \operatorname{span}$; (a) $t / \tau_{r}=0.0$, (b) $t / \tau_{r}=0.5$; dashed lines indicate likely rotor shock locations. From Chang and Tavoularis (2009b).

The pressure contours allowed for the identification of the approximate locations of rotor trailing edge shocks: one shock is evident near the trailing edge of blade $M$ on the $10 \%$ spanwise plane at $t / \tau_{r}=0.0$ and another near the trailing edge of blade $L$ at $t / \tau_{r}=0.5$

The distribution of relative Mach number (contours shown in Fig. 7.15) clearly indicates that a shock appeared at the $10 \%$ span plane but not at the $50 \%$ span plane. This may be partially attributed to the transportation of low momentum, high loss fluid from the rotor hub to the middle span of the rotor blade by the rotor hub passage vortex (Fig. $7.8 \mathrm{~b})$, thus reducing the flow velocity near the mid-span of the rotor.

The contours of static pressure at the outlet plane of the stator, plotted in Fig. 


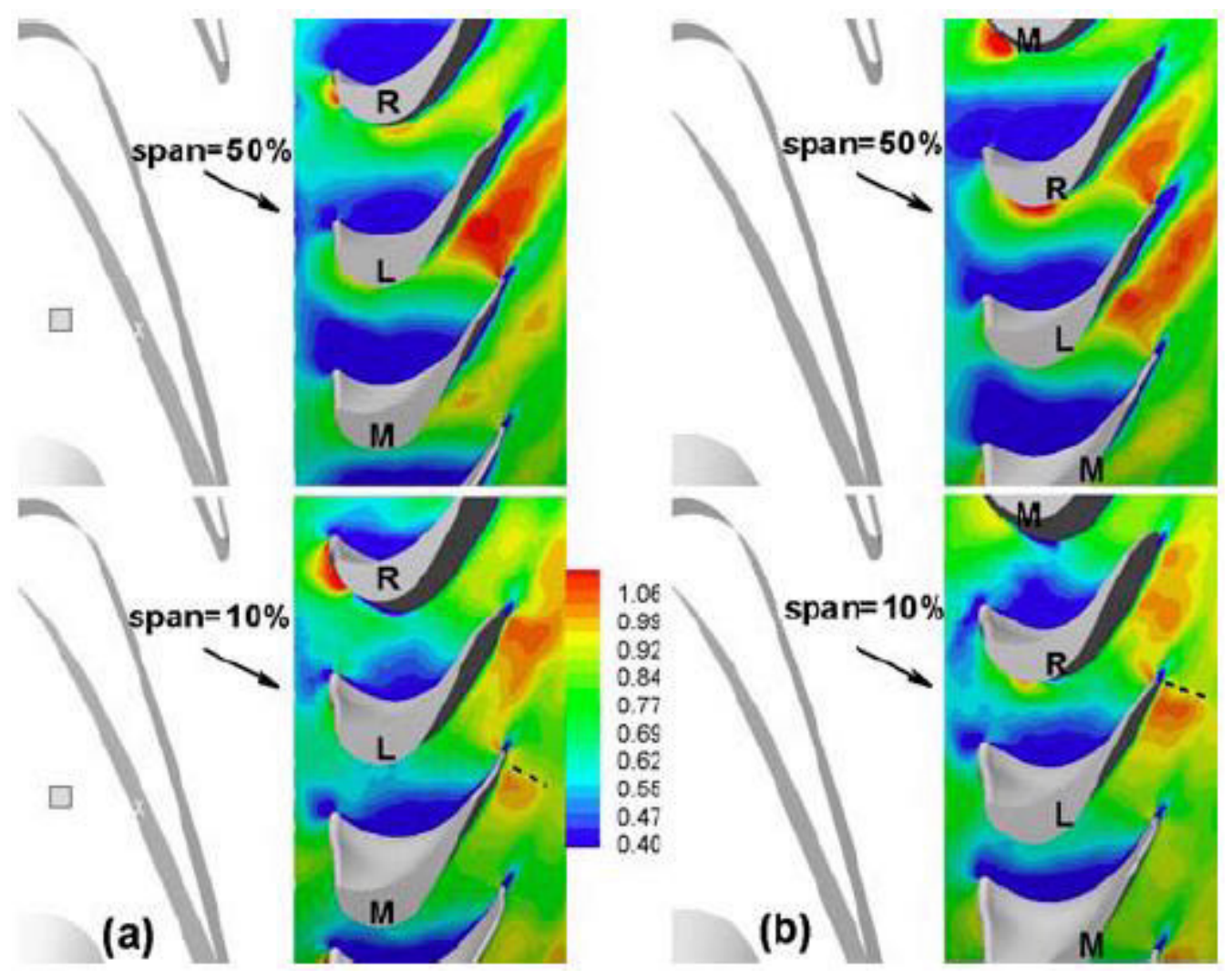

Figure 7.15: Instantaneous contours of relative Mach number on two planes in the flow, at $50 \%$ and $10 \%$ span, and at times (a) $t / \tau_{r}=0.0$, and (b) $t / \tau_{r}=0.5$; hand-drawn dashed lines indicate possible rotor shock locations. From Chang and Tavoularis (2009b). 
7.16, indicate that local low-pressure regions (marked by hand-drawn circles) were formed near the hub endwall on the vane outlet plane. These local low-pressure regions accelerated the flow, also contributing to the formation of rotor trailing edge shocks near the hub, as shown in Fig. 7.15. This may also be inferred by comparing Fig. 7.16 with Fig. 7.17, which shows the static pressure distribution on a plane normal to the turbine axis, downstream of the trailing edges of the rotor blades by a distance equal to $10 \%$ of the axial blade chord. The shocks near the hub section of the blade trailing edge, marked by dashed lines in Fig. 7.17, seem to be associated with the presence of low-pressure cores, marked by circles in Fig. 7.16, at the stator outlet.

Based on these observations, one may conclude that the potential field upstream of the blades affected the flow not only as it entered the blade passage, but also in the region downstream of the rotor passage.

\subsubsection{Wakes}

Figure 7.18 illustrates the instantaneous entropy distribution on two planes in the flow, at $50 \%$ and $10 \%$ span, and two time steps. Although the shapes of the contours changed significantly from one instant to the next, the wake patterns were consistent at both times: the wake of each vane was first compressed as it impinged on a rotor blade; then it migrated from the pressure side of one blade towards the suction side of the next on-coming blade (e.g., from blade $R$ to blade $M$ in Fig. $7.18 \mathrm{~b}$ at $50 \%$ span); finally, the vane wake joined the wake of a blade (Casciaro et al. (2000)). The strength of the vane wakes seemed to be consistent until they join the blade wakes. In contrast to vane wakes, which were stronger near the hub (10\% span) than at midspan ( $50 \%$ span), rotor wakes 

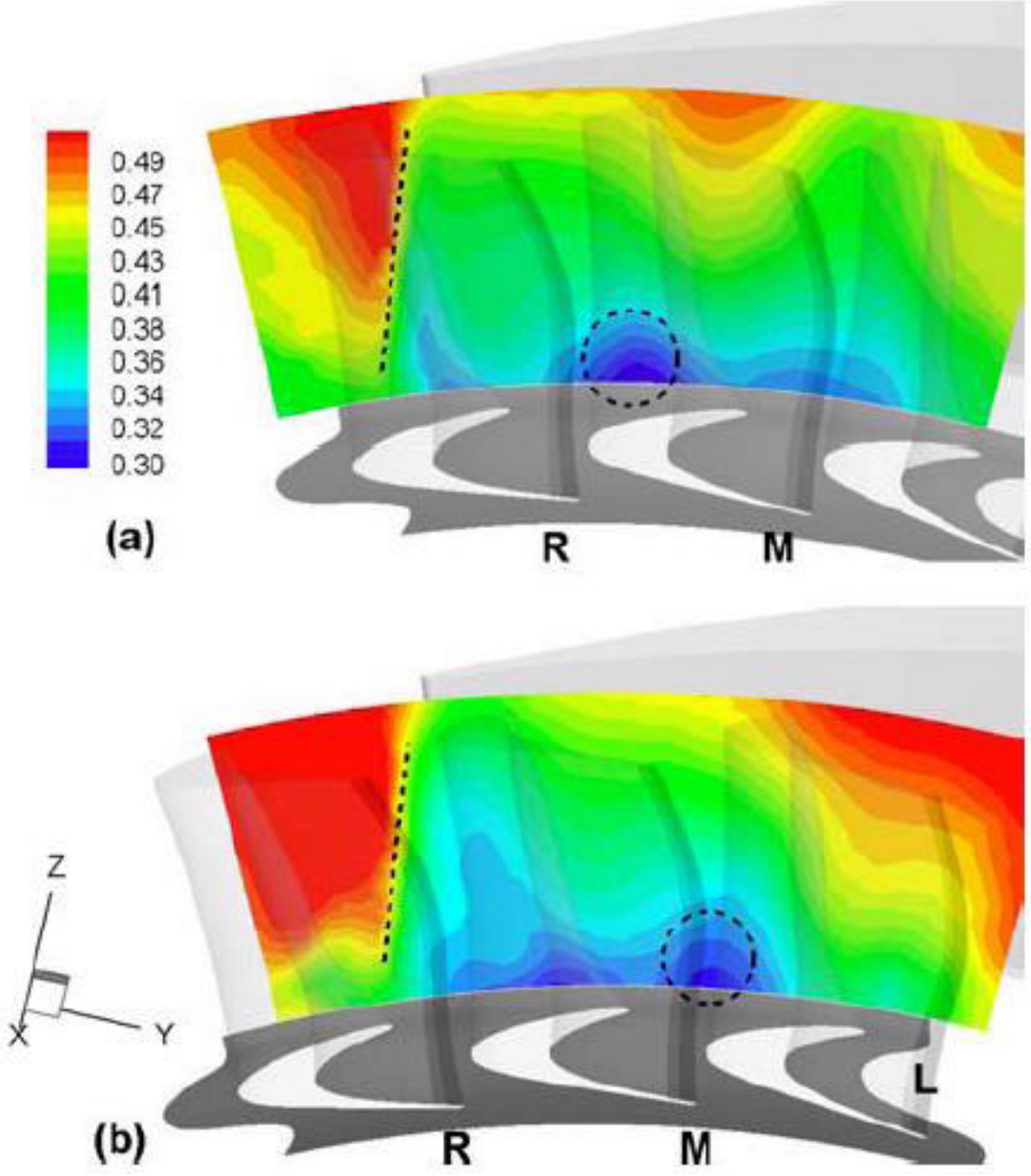

Figure 7.16: Contours of static pressure normalized by the total inlet pressure on the stator outlet plane at times (a) $t / \tau_{r}=0.0$, and (b) $t / \tau_{r}=0.5$; hand-drawn dashed circles emphasize a local low-pressure region, dashed lines mark approximate locations of vane trailing edge shocks, and view is from downstream. From Chang and Tavoularis (2009b). 

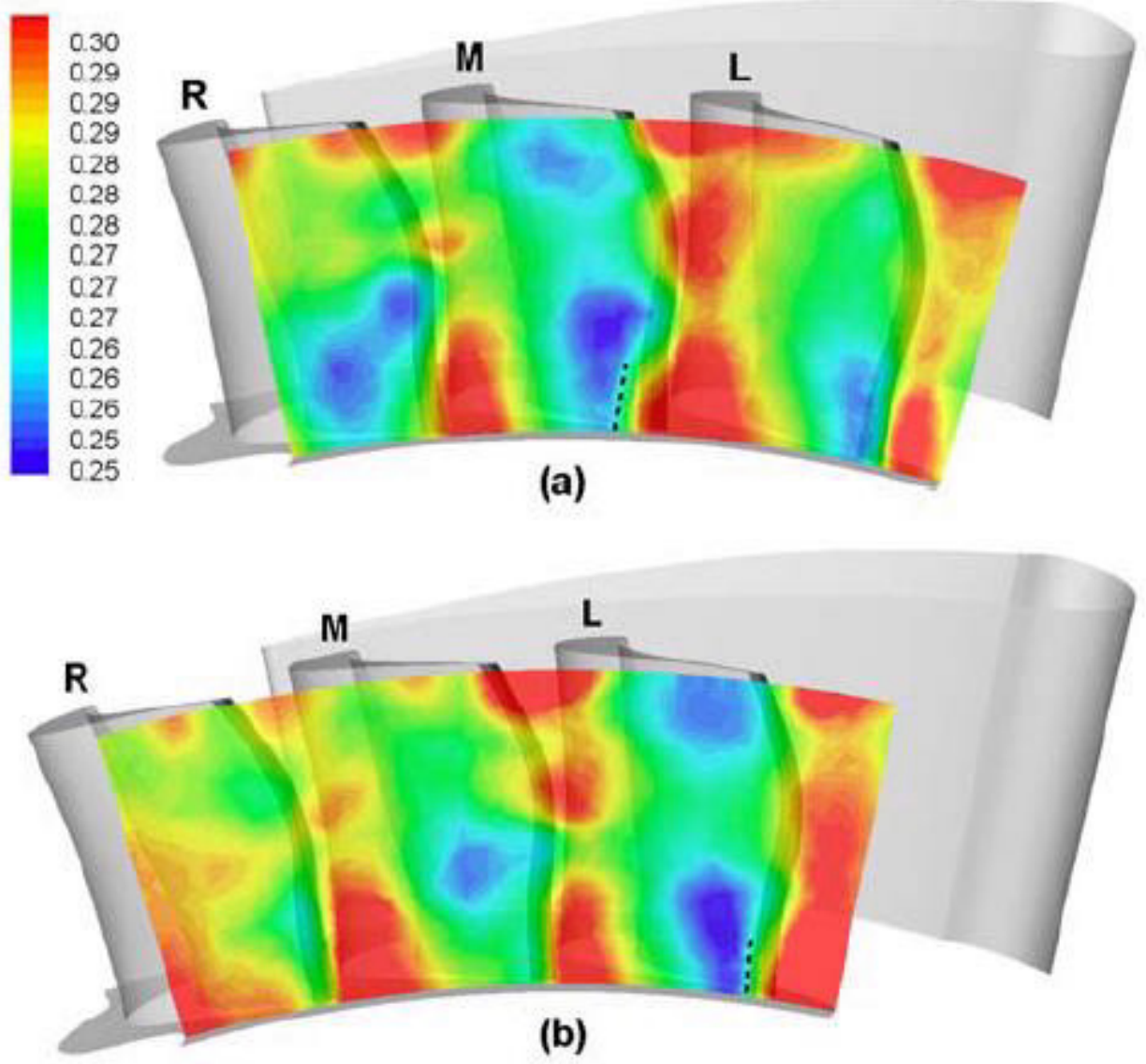

Figure 7.17: Contours of static pressure normalized by the total inlet pressure on a plane normal to the turbine axis at an axial location that is downstream of the rotor blade trailing edges by a distance equal to $10 \%$ of the axial blade chord, at times (a) $t / \tau_{r}=0.0$, and (b) $t / \tau_{r}=0.5$; view is from downstream and dashed lines mark possible blade trailing edge shocks. From Chang and Tavoularis (2009b). 
were stronger at midspan than at $10 \%$ span.
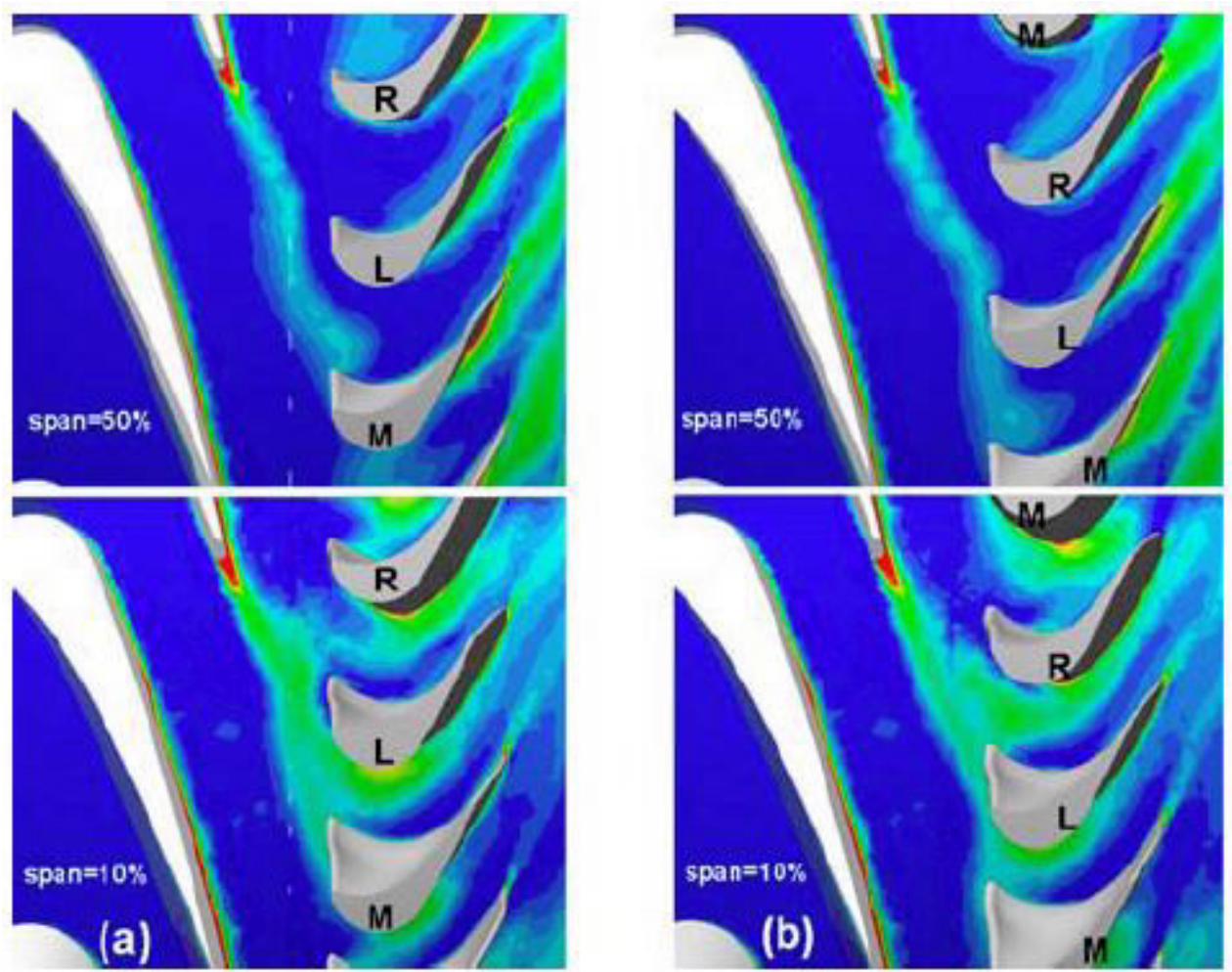

Figure 7.18: Instantaneous entropy contours on two planes in the flow, at $50 \%$ and $10 \%$ span and times (a) $t / \tau_{r}=0.0$, and (b) $t / \tau_{r}=0.5$. From Chang and Tavoularis $(2009 \mathrm{~b})$.

Inspection of total pressure maps downstream of the rotor (not shown here) revealed that pressure losses were higher near the midspan of the rotor outlet than towards the endwalls. Such losses are mainly attributed to the rotor blade wakes (Denton (1993)). This is also illustrated in Fig. 7.19, which shows contours of entropy on a plane normal to the turbine axis at an axial location that is $10 \%$ of the axial blade chord downstream of the rotor blade trailing edges. 

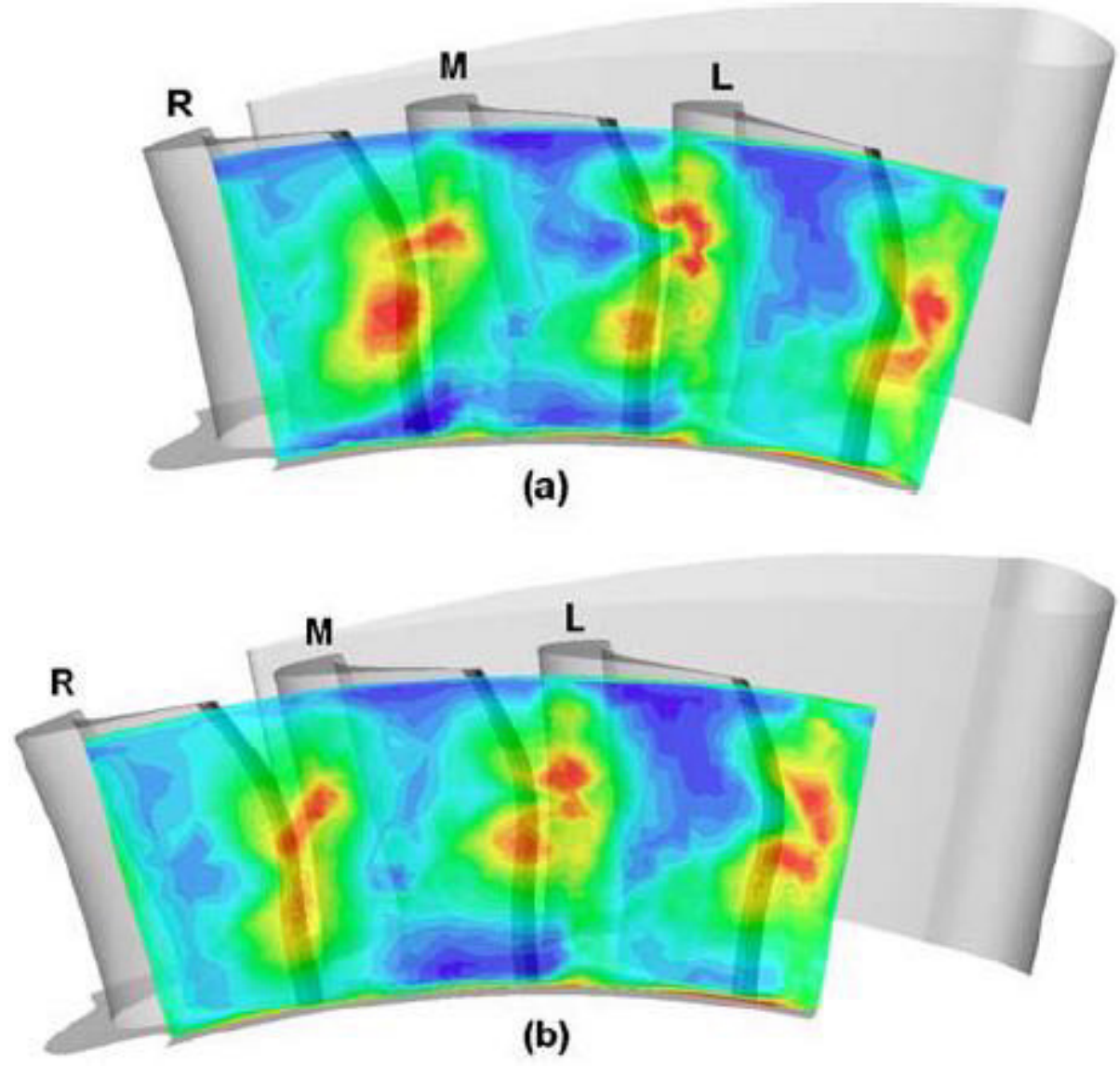

Figure 7.19: Contours of entropy on a plane normal to the turbine axis at an axial location that is downstream of the trailing edges of the rotor blades by a distance equal to $10 \%$ of the axial blade chord, at times (a) $t / \tau_{r}=0.0$, and (b) $t / \tau_{r}=0.5$; view is from downstream. From Chang and Tavoularis (2009b). 


\subsection{Summary}

In performing the simulation of the turbine stage with a uniform inlet temperature distribution, emphasis was placed on identifying and characterizing the following important flow features: large-scale vortices, which have been identified with the use of the modified $Q$ criterion; shock waves in the stator and the rotor, identified as surfaces across which pressure rises sharply; and the wakes of vanes and blades, identified as regions with relatively high entropy.

The sizes, senses of rotation, and strengths of well-known quasi-stationary vortices have been determined and clearly visualized. These structures include the vane casing horseshoe and passage vortices, blade casing and hub horseshoe and passage vortices, blade tip leakage vortices, and vortices shed by the trailing edges of vanes and blades. Blade passage vortices were shown to create low pressure regions on the suction side of the blade and to transport low momentum fluid from the end-walls toward the blade mid-section. A previously undocumented type of vortex, termed the axial gap vortex, was identified during part of the blade passing period, and has been associated with the interactions between vane wakes and passing blades. A second type of hitherto unknown vortex, termed the crown vortex, has been detected in the mid-section of the blade passage near the location of the blade crown.

Shock waves originating from the vane trailing edges were shown to be responsible for large surface pressure fluctuations on the upstream portion of the suction side of the blade. Shock waves have also been observed near the blade trailing edges, primarily near the hub; these shocks have been associated with local low pressure regions in the flow at 
the vane outlet, whereas the absence of shocks in the blade midspan has been associated with spanwise transport of low momentum fluid by the passage vortices. The observed high total pressure losses in the turbine at blade midspan have also been connected to the action of passage vortices.

The trends and phenomena discussed in this chapter were found to be consistent with those found in the subsequent simulations, which include non-uniform inlet temperature distributions. 


\section{Chapter 8}

\section{Hot Streak Simulation Results and}

\section{Discussion}

This chapter presents and discusses the results of the simulations using the vaneimpinging (VI) and mid-pitch (MP) non-uniform inlet fields. The $V I$ case the one in which hot streaks impact directly on the leading edge of the stator vanes. In the $M P$ case, hot streaks pass between the stator vanes.

\subsection{In-Flow Property Variation}

In this section, the focus is on the direct effects of the hot streaks as they progressed through the turbine at roughly $70 \%$ span. At this spanwise location, the hot streak influence was determined to be strongest for both hot streak configurations. Instantaneous contours of normalized total enthalpy on a plane slightly upstream of the vane trailing edges are shown in Fig. 8.1, and instantaneous contours of relative total enthalpy (i.e., based on the 
flow velocity relative to the rotating frame of the rotor) in a plane slightly downstream of the leading edges of the rotor blades are shown in Fig. 8.2.

As seen in Fig. 8.1a, the VI case, hot gas in the portion of the hot streak passing along the vane pressure side was entrained into the stator casing passage vortex (see section 7.2.2), whereas the rest of the hot gas shifted towards the hub, in response to the strong radial pressure gradient near the pressure surface of the vane. In the $M P$ case, no significant hot streak migration was noticeable.

For the time step shown in Fig. 8.2, the vane-impacting hot streak entered the rotor passage and impinged on the leading edge of the leftmost blade, where it was split for a second time. A patch of low-enthalpy fluid passed over the suction side of the rightmost blade, with a smaller portion passing toward the pressure side. This cool streak originated from the cooler fluid between hot streaks. In the mid-pitch hot streak case, the hot streak entered the rotor section between the middle and rightmost blades without much spanwise shifting, in agreement with the findings of Qingjun et al. (2010).

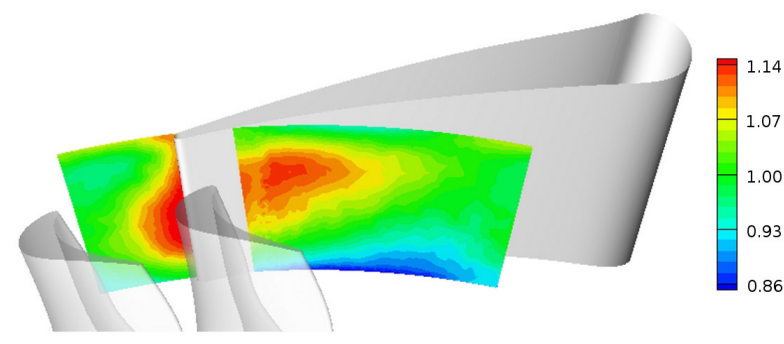

(a)

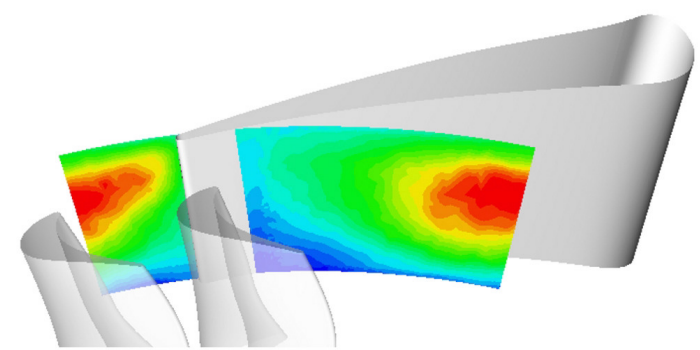

(b)

Figure 8.1: Instantaneous contours of total enthalpy in a plane slightly upstream of the vane trailing edge for the (a) $V I$ and (b) $M P$ cases, normalized by $h_{o 1}$.

Comparison of the results for the two hot streak configurations reveals significant differences in the enthalpy distributions through both the stator and the rotor. Figure 


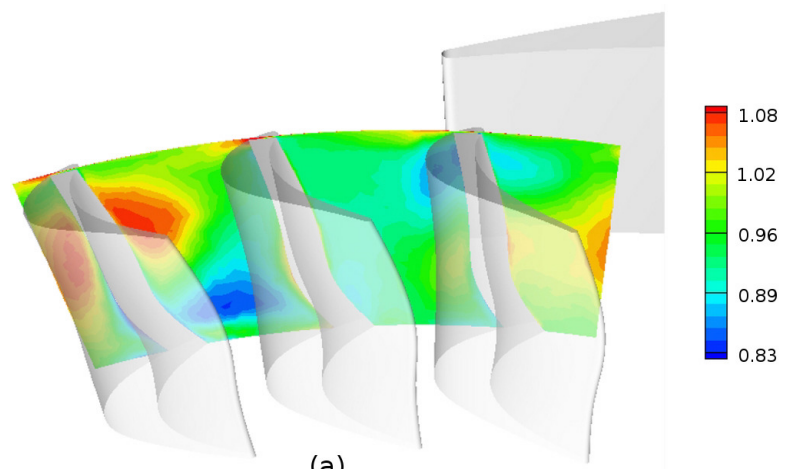

(a)

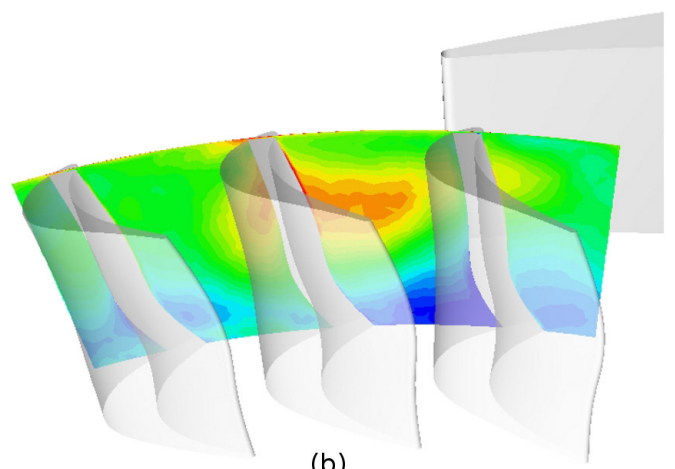

(b)

Figure 8.2: Instantaneous contours of relative total enthalpy in a plane slightly downstream of the rotor passage entrance for the (a) VI and (b) MP cases, normalized by $h_{o 1}$.

8.3 shows instantaneous total enthalpy contours for the $V I$ case at $70 \%$ span, as the gas flowed through the turbine. Figure 8.3a illustrates an instant during which the wake of a vane was aligned with a blade, whereas Fig. $8.3 \mathrm{~b}$ illustrates an instant half a blade passing period later. Contours are shown for total enthalpy in the stator section and for relative total enthalpy in the rotor section. Note that the total and relative total contour levels in Figs. 8.3 and 8.4 were chosen to provide visual continuity across the interface. The $V I$ hot streak clearly surrounded the stator vane, and through spanwise redistribution, lost much of its strength at this location by the time it reached the stator-rotor axial gap. Although a distorted hot streak visibly impinged on the blade's leading edge in Fig. 8.3a, it was effectively split by the chopping action of the rotor blades, with its strength diminishing until it was barely distinguishable at the rotor exit. Whereas the hot streaks were split by the blades, the cooler fluid was clearly drawn towards the suction sides of the blades, demonstrating the well-known segregation effect (Butler et al. (1989)). This cooler fluid did not mix thoroughly with the surrounding fluid, remaining coherent at the rotor's exit.

Figure 8.4 shows instantaneous contours for the $M P$ hot streak case, with the 


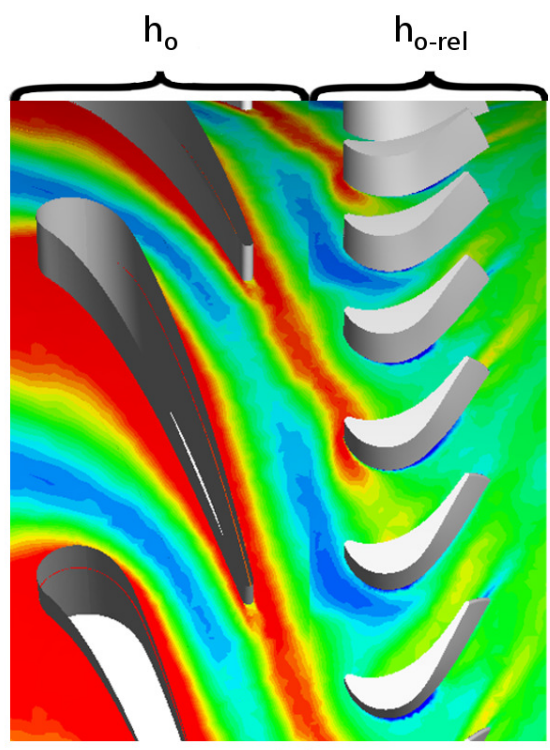

(a)

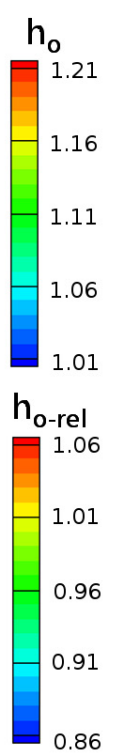

0.86

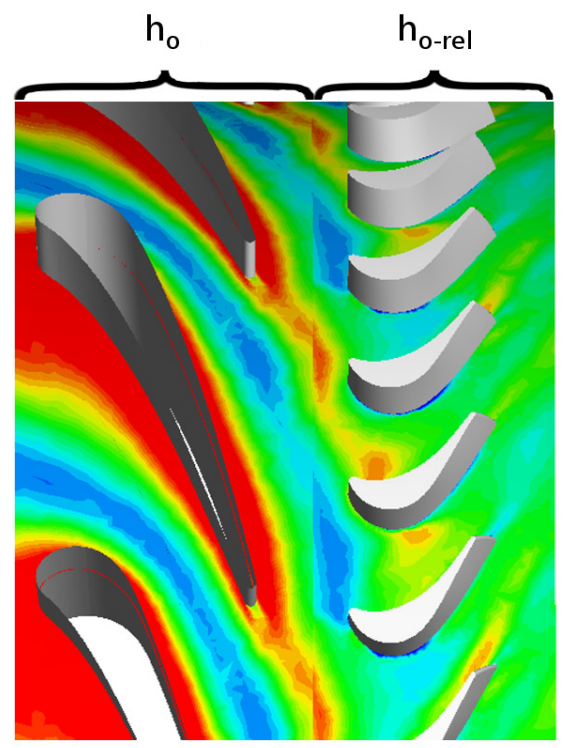

(b)

Figure 8.3: Instantaneous contours of the dimensionless total enthalpy $h_{o} / h_{o 1}$ and relative total enthalpy $h_{o-r e l} / h_{o 1}$ at $70 \%$ span for the $V I$ configuration at times (a) $t / \tau_{r}=0.0$, and (b) $t / \tau_{r}=0.5$.

same stator-rotor alignments as in Fig. 8.3. The hot streak maintained much of its strength through both the stator and the rotor, and the enthalpy in the axial gap region was distinctly non-uniform. Figure 8.4b particularly shows the accumulation of high-enthalpy fluid in the passage between two blades, before being convected downstream. The hot streaks remained fairly coherent and maintained their strength through the rotor section.

In general, the VI configuration had a more uniform enthalpy distribution in the rotor section, with a predominantly mid-range enthalpy at $70 \%$ span, whereas the rotor section in the $M P$ case had alternating regions of higher and lower enthalpy at this spanwise location. This makes the $V I$ configuration preferable for the purposes of designing the low-pressure turbine and predicting its operation, as the more even distribution would approximately conform to the commonly employed assumption of uniform inlet conditions. 


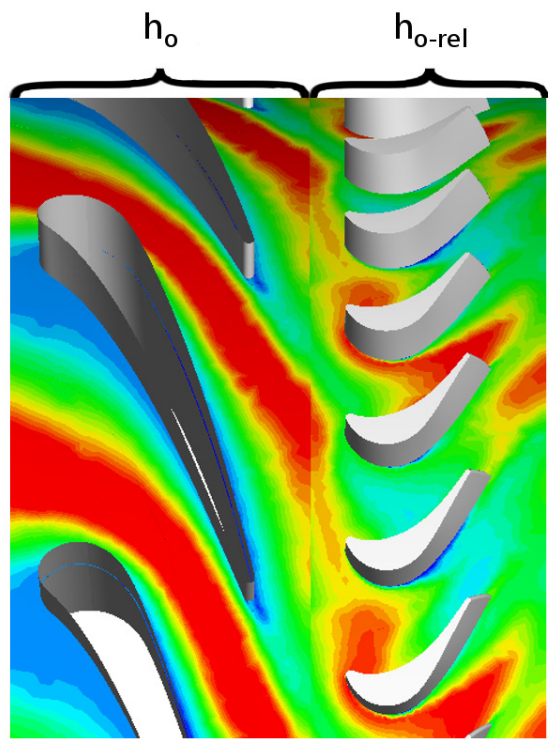

(a)

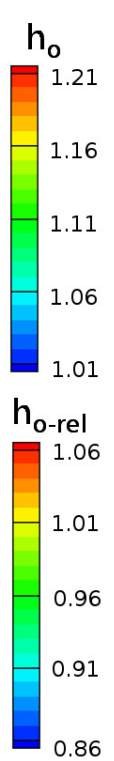

0.86

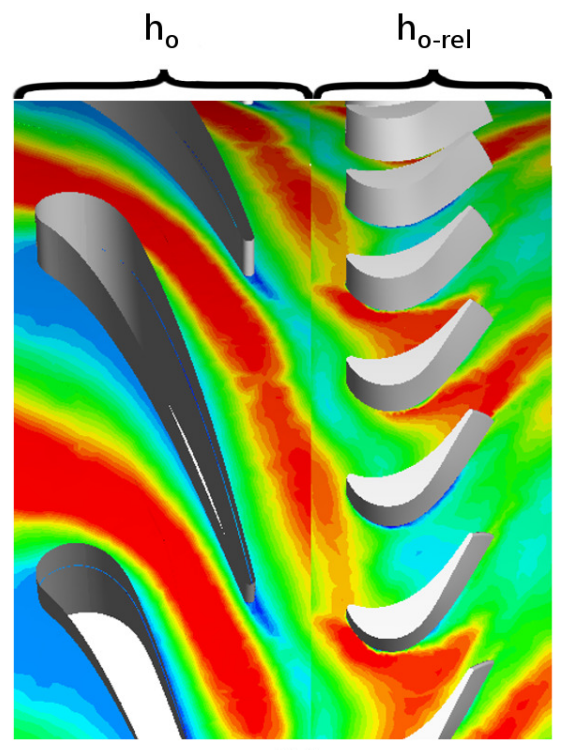

(b)

Figure 8.4: Instantaneous contours of the dimensionless total enthalpy $h_{o} / h_{o 1}$ and relative total enthalpy $h_{o-r e l} / h_{o 1}$ at $70 \%$ span for the $M P$ configuration at (a) $t / \tau_{r}=0.0$ and (b) $t / \tau_{r}=0.5$

Figure 8.5 shows pressure contours at $70 \%$ span for the $V I$ configuration. Figure 8.5 is representative of all studied cases, as the pressure contours were nearly identical for the $V I, M P$, and reference cases. The main feature of note is the location of the vane trailing edge shock, indicated by a dashed line in the figure.

In Fig. 8.5a, the downstream shock passed through the middle of a blade passage, whereas in Fig. 8.5b the shock impinged on the leading edge of the nearby blade. The behaviour and effects of pressure and shock patterns are very similar to those in the reference case (Chang and Tavoularis (2009a)). A point of note is that although the shock's effects are observed primarily near the leading edge of the blade suction side, the interaction extends further into the rotor passage, affecting the entire pressure side to some degree. Also note the presence of the vane's passage shock, indicated by the discontinuity between the upper 


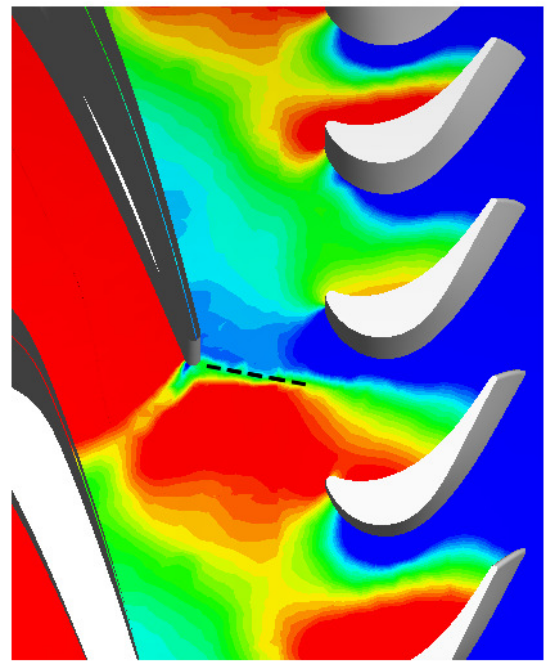

(a)

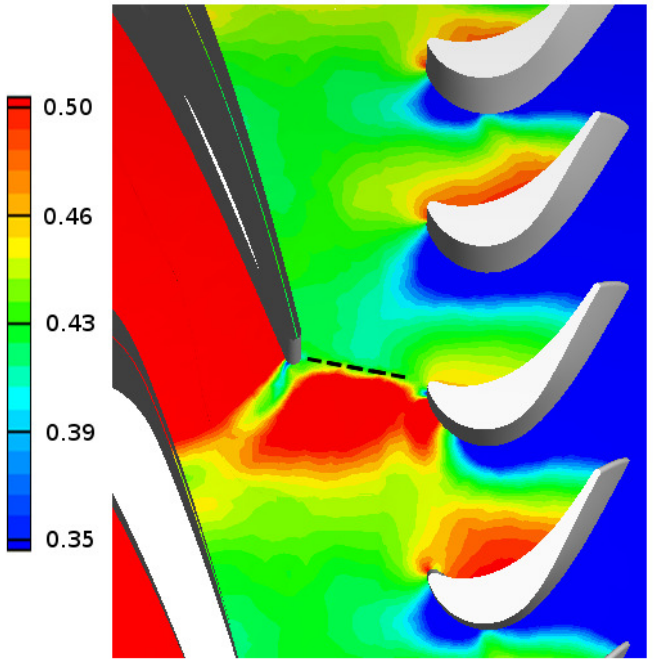

(b)

Figure 8.5: Instantaneous contours of pressure at 70\% span at (a) $t / \tau_{r}=0.0 t / \tau_{r}=0.0$ and (b) $t / \tau_{r}=0.5$, normalized by $P_{o 1}$. Dotted line indicates shock location.

vane's trailing edge and the mid-chord suction side of the lower vane.

Although the use of contours of total temperature or enthalpy to indicate the effects of the hot streak is common practice (e.g., Qingjun et al. (2010)), contours of entropy more clearly illustrate the paths of the hot streaks through the turbine. Figure 8.6 shows instantaneous contours of constant entropy for the $V I$ case, again for the same two stator-rotor alignments. The hot streak was seen clearly to move away from the vane trailing edge and enter the rotor section, where it was chopped and convected downstream, while the regions of lower entropy remained largely intact as they passed over the suction side.

Entropy contours for the $M P$ case are shown in Fig. 8.7. They indicate a comparatively wide hot streak exiting the stator section, as well as less low-entropy fluid between hot streaks. The effect of segregation on the hot streaks was evident in this case, and the 


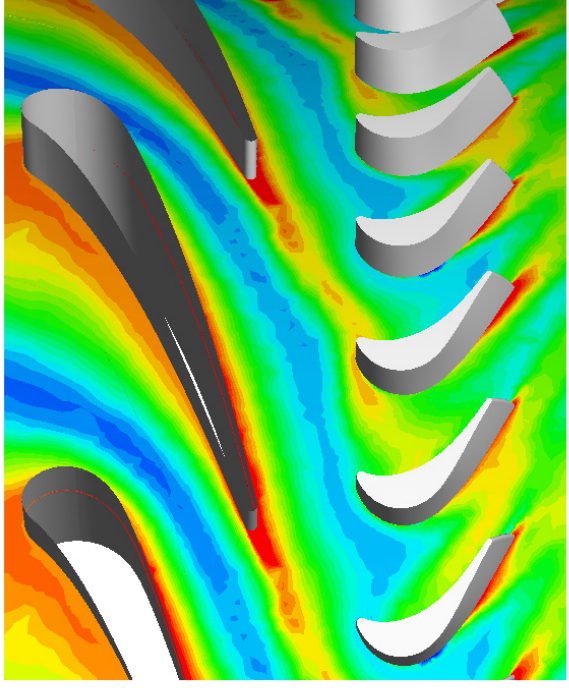

(a)

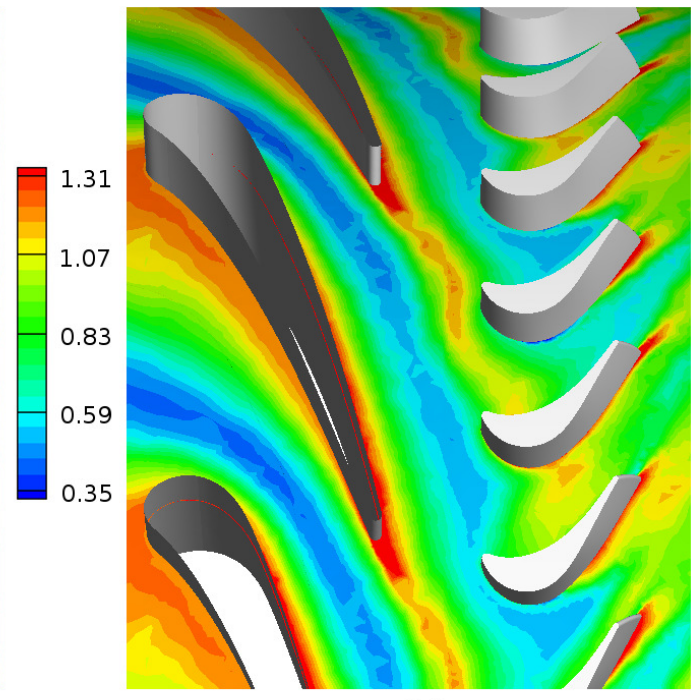

(b)

Figure 8.6: Instantaneous contours of entropy at $70 \%$ span for the $V I$ configuration at (a) $t / \tau_{r}=0.0$, and (b) $t / \tau_{r}=0.5$, normalized by $\mathbf{R}$.

regions of lower entropy were not apparent, following mixing with the stator vane's wake.

Note that, in Figs 8.6 and 8.7, the high-entropy fluid in the blade wakes should not be confused with hot streak effects, although the instantaneous contours of total enthalpy and entropy are similar in pattern for each hot streak configuration.

Traditionally, the design of gas turbines has been largely based on time-averaged flow properties. Figure 8.8 shows contours of time-averaged total enthalpy in the stator and relative total enthalpy in the rotor. The rotor section in the $M P$ case (Fig. 8.8b) clearly experienced significantly higher enthalpy than in the VI case (Fig. 8.8a). The highest levels of relative total enthalpy in the rotor section appeared near the pressure side for the $V I$ case, and over the entire forward section for the $M P$ case. The relative total enthalpy at the rotor exit was substantially higher at this spanwise location in the $M P$ case, although the mass-weighted, time-averaged values are not significantly different. 


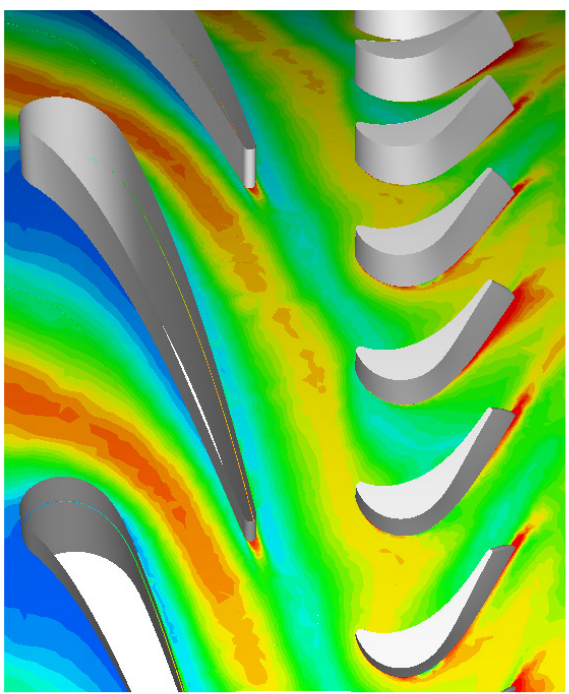

(a)

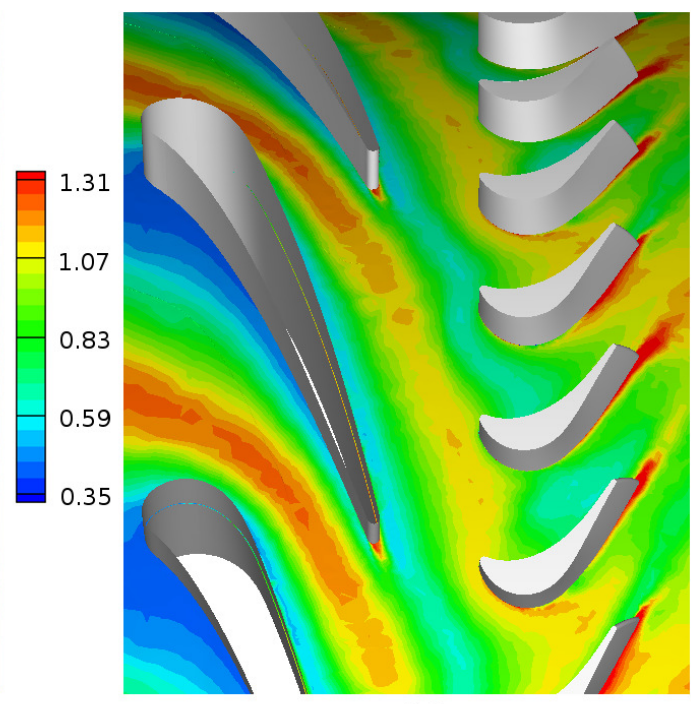

(b)

Figure 8.7: Instantaneous contours of entropy at $70 \%$ span for the $M P$ configuration at (a) $t / \tau_{r}=0.0$, and (b) $t / \tau_{r}=0.5$, normalized by $\mathbf{R}$.

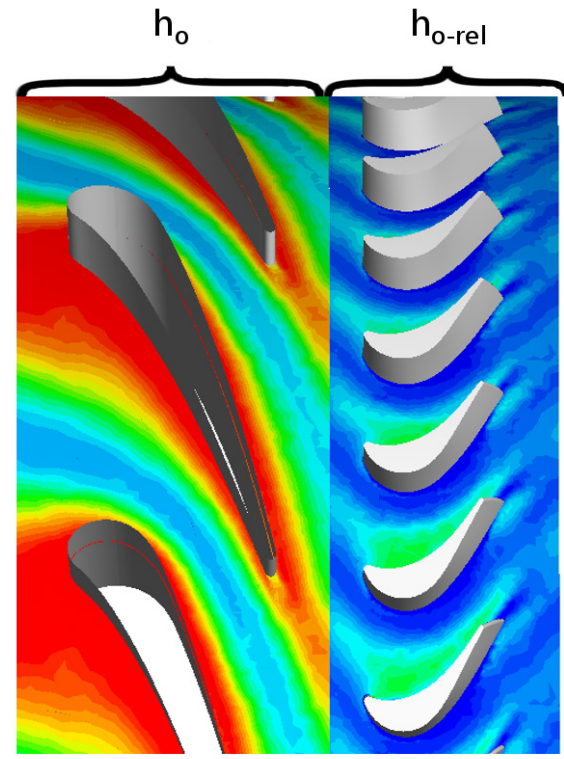

(a)

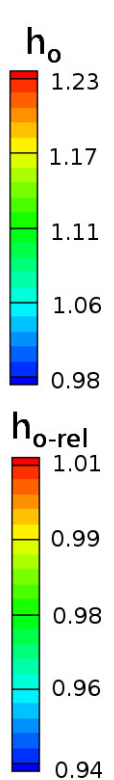

0.94

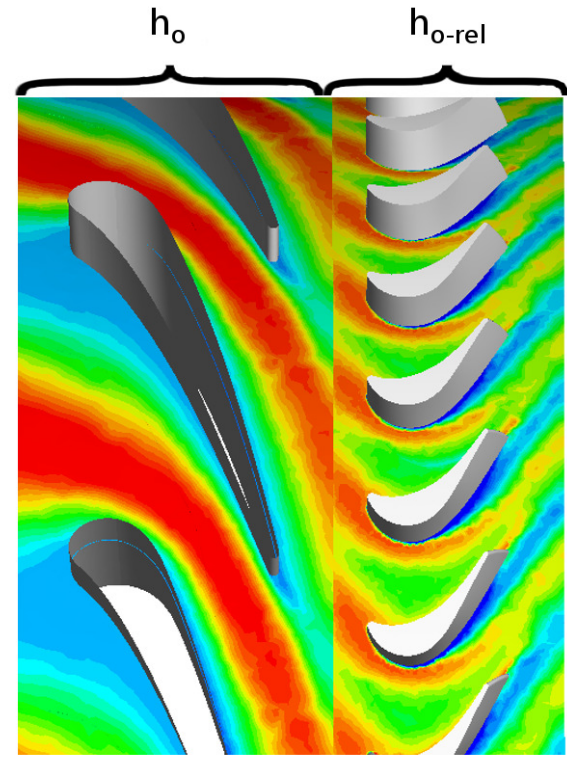

(b)

Figure 8.8: Time-averaged contours of total enthalpy and relative total enthalpy at $70 \%$ span for the (a) VI and (b) $M P$ cases, normalized by $h_{o 1}$. 
The standard deviation of the relative total enthalpy in the rotor section is shown in Fig. 8.9. Near the leading edge, the high fluctuations seen in the $V I$ case were the result of the alternating high- and low-enthalpy fluid passing through this region from the stator section (Figs. 8.2a and 8.3). The variation in the $M P$ case was lower, as the enthalpy varied from a high level to a moderate one (Figs. 8.2b and 8.4). Note that the stator section was omitted from Fig. 8.9, as it shows relatively very little deviation.

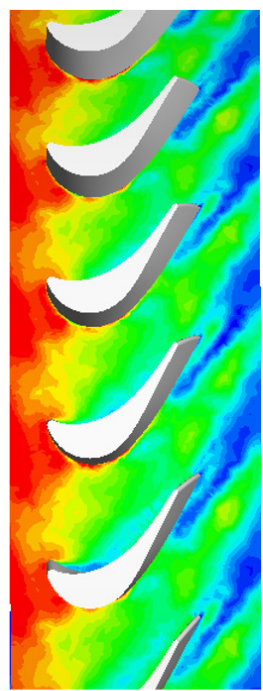

(a)

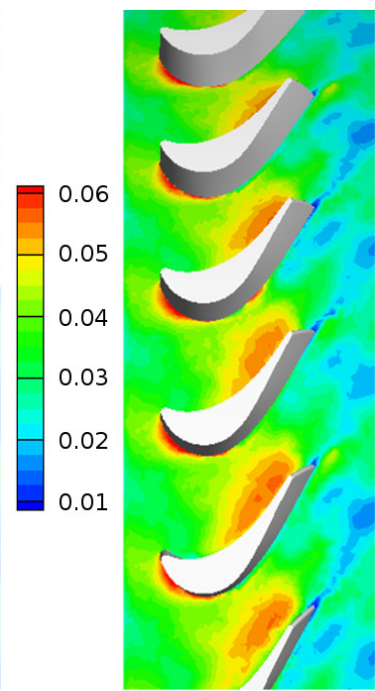

(b)

Figure 8.9: Rotor section contours of the standard deviation of relative total enthalpy at $70 \%$ span for the (a) VI and (b) $M P$ cases, normalized by $h_{o 1}$.

The strong enthalpy fluctuations near the trailing edges of the pressure sides of the blades in the $M P$ configuration can be attributed to the segregation of hot streaks and their subsequent replacement by moderate-enthalpy fluid. At the same location in the VI case, enthalpy fluctuations were significantly lower, because the strengths of the hot streaks were largely diminished, both by impact on the vanes, and through the chopping of the blades and mixing with cooler fluid. At this spanwise position, the enthalpy fluctuation levels at 
the rotor exit were also visibly lower for the $V I$ case.

Contours of time-averaged entropy in the rotor section are presented in Fig. 8.10; time-averaged contours in the stator section are not shown as they are very similar to the instantaneous contours. The fluid in the rotor for the $M P$ configuration at this spanwise position had relatively high entropy, due to its mixing with fluid from the wake of the vane. The high entropy fluid near the downstream half of the suction side for both configurations was unrelated to the hot streak, but likely associated with flow separation from the blades.

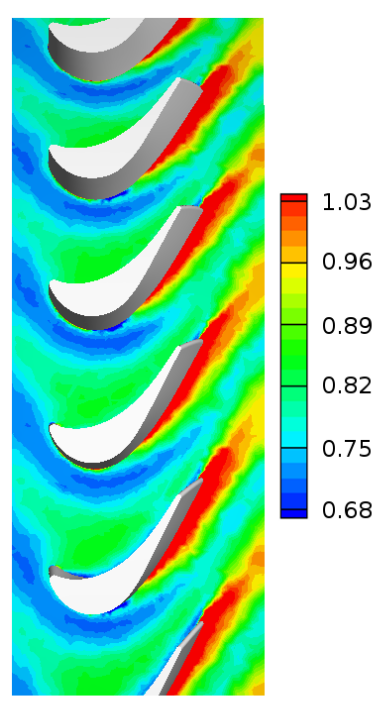

(a)

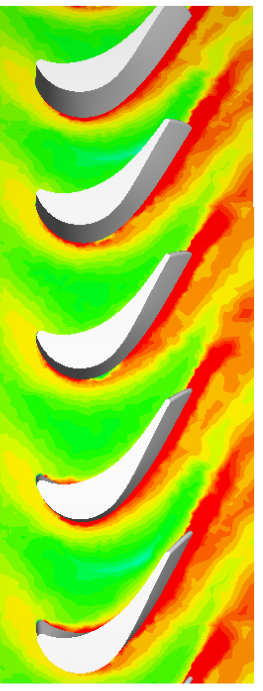

(b)

Figure 8.10: Time-averaged contours of entropy in the rotor at $70 \%$ span for the (a) $V I$ and (b) $M P$ cases, normalized by $\mathbf{R}$.

The standard deviation of the entropy at $70 \%$ span is shown in Fig. 8.11. Entropy fluctuations at the rotor inlet and throughout the blade passages were stronger in the $V I$ case, for which regions of high- and low-entropy fluid alternated in time (Fig. 8.6). In the $M P$ case, upstream mixing of high-entropy fluid from the vane wake with low-entropy, cooler fluid (Fig. 8.7) reduced entropy fluctuations in the rotor section. 


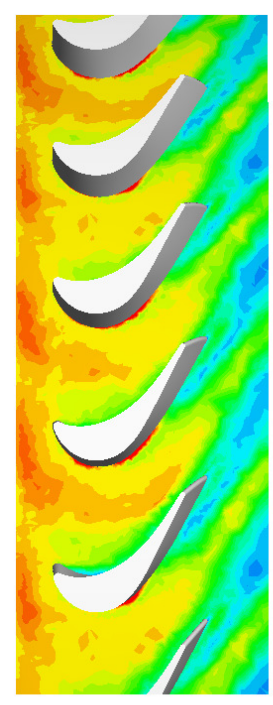

(a)

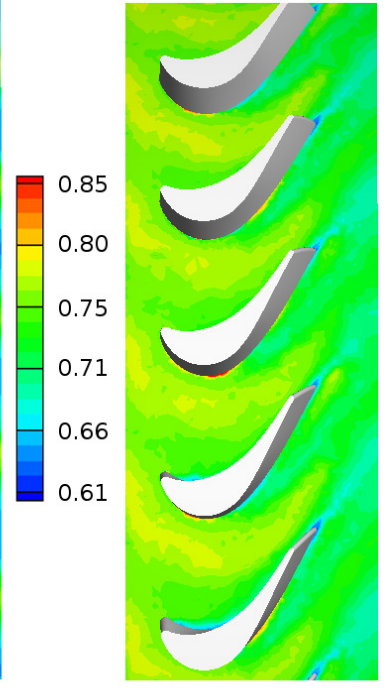

(b)

Figure 8.11: Contours of entropy standard deviation in rotor at $70 \%$ span for the (a) VI and (b) $M P$ cases, normalized by $\mathbf{R}$.

\subsection{Surface Property Variation}

\subsubsection{Vane Surfaces}

Figure 8.12 shows contours of time-averaged enthalpy on the suction and pressure surfaces of the vane. The VI case showed evidence of the hot streak flowing over both sides, though predominantly enveloping the pressure side. On the pressure side, the influence of the hot streak was very strong over most of the vane's span, except close to the hub; on the suction side this influence was more visible around the vane midspan. Spanwise spreading of the hot streak on the pressure side may be attributed to the combined actions of vane passage vortices and radial pressure gradient. In the $M P$ case, the vane was clearly cooler than in the aligned case.

The enthalpy standard deviation on the vane suction side is shown in Fig. 8.13, 

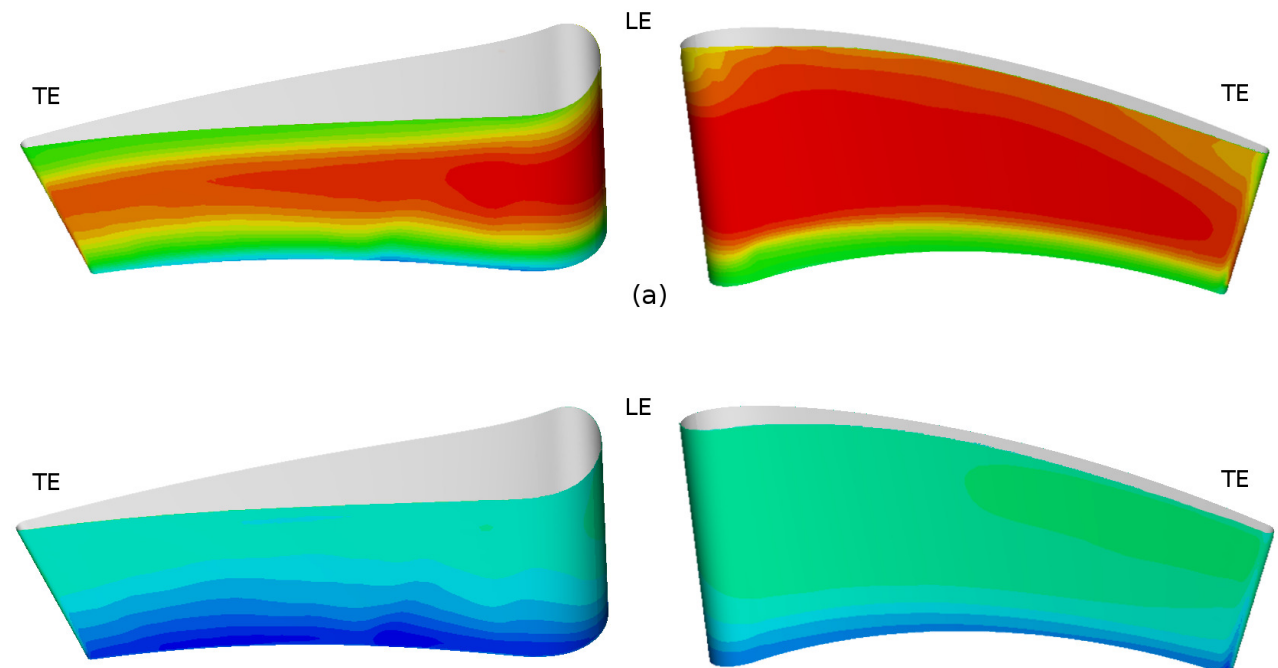

LE

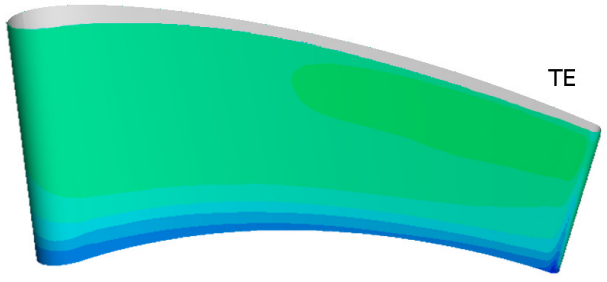

(b)

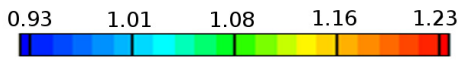

Figure 8.12: Time-averaged static enthalpy contours on the vane surfaces for the (a) VI and (b) $M P$ cases, normalized by $h_{o 1}$.

for both hot streak configurations. The fluctuation levels were much lower on the pressure side and are not shown here. Both cases had similar patterns, with the highest fluctuations occurring near the trailing edge and towards the hub, although the high-fluctuation area was wider in the $V I$ case. Fluctuations increased suddenly near the $30 \%$ chord line, which was slightly downstream of the location of impingement of the passage shock originating from the neighbouring vane's trailing edge (Fig. 8.4).

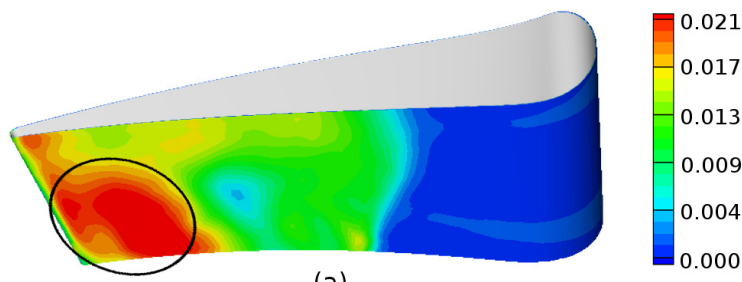

(a)

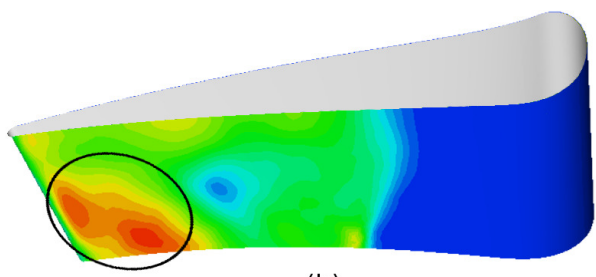

(b)

Figure 8.13: Contours of static enthalpy standard deviation on the vane suction side for (a) the $V I$ and (b) $M P$ cases, normalized by $h_{o 1}$. 


\subsubsection{Blade Surfaces}

Contours of the time-averaged blade surface enthalpy are shown in Fig. 8.14. On the pressure side, the enthalpy was higher in the $M P$ case and its peak was closer to the tip. On the suction side, enthalpy peaks occurred near the tip and towards the trailing edge for both configurations, with a stronger peak for the $M P$ case, likely due to tip leakage flow. Two pairs of small maxima and minima were seen in each case near the mid-chord of the suction side, one pair near the root and a second one near the tip. These corresponded to the locations of the casing and hub passage vortices (see section 7.2.2). In both the $V I$ and $M P$ cases, but not in the reference case (not shown here), one may notice some striations of lower enthalpy near the trailing edge of the blade pressure side. The cause of these striations is not clear.

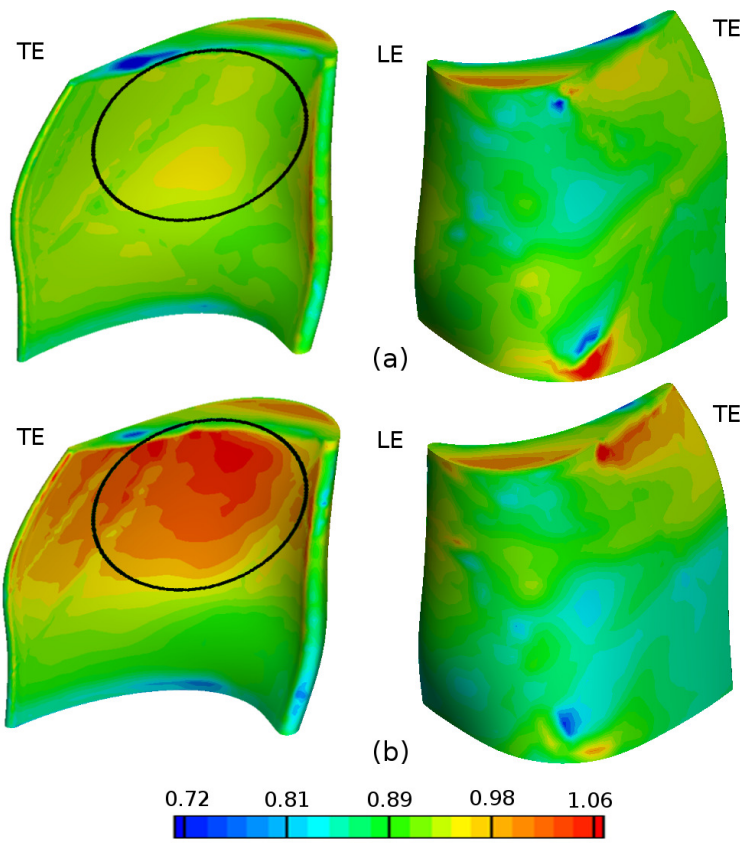

Figure 8.14: Time-averaged static enthalpy contours on the blade surfaces for (a) the $V I$ and (b) $M P$ cases, normalized by $h_{o 1}$. 
Figure 8.15 shows the enthalpy standard deviation on the blade surfaces. The suction side contours show the effects of shock interaction. The dashed lines mark locations of peak pressure fluctuations (not shown presently), which are indicative of strong influence of the vane trailing edge shock waves, also generating strong enthalpy fluctuations. Below the midspan of the blade suction side, the enthalpy fluctuations were slightly stronger in the $V I$ case, due to the spanwise spreading of the hot streak (Fig. 8.2).

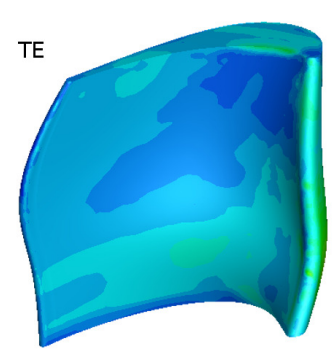

LE
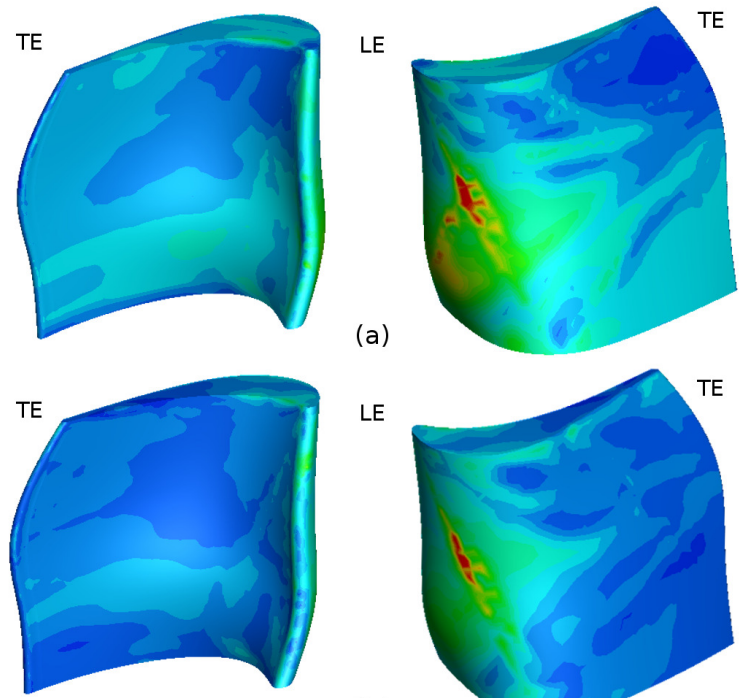

(a)
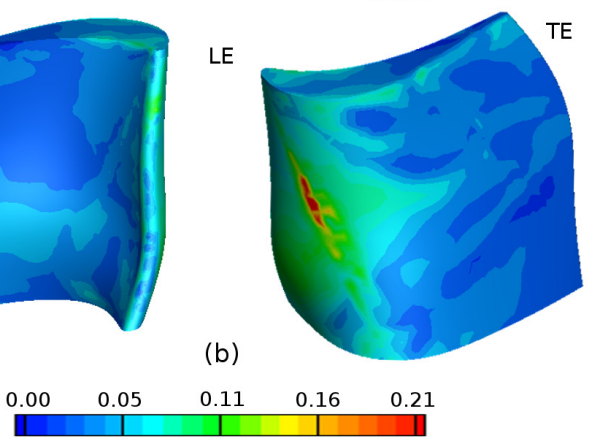

Figure 8.15: Contours of static enthalpy standard deviation on the blade surfaces for the (a) VI and (b) $M P$ cases, normalized by $h_{o 1}$.

Contours for the blade surface time-averaged pressure and its standard deviation showed very small differences between each of the two hot streak configurations and the reference case, in contrast to the finding of An et al. (2009). Thus, Fig. 8.16 is representative of the three cases of concern in this study. In a previous publication (Chang and Tavoularis (2009a)), the formation of local low-pressure regions on the suction sides of the blades has 
been described, which indicate the presence and locations of tip leakage vortices and hub and casing passage vortices, as well as the crown vortices, as mentioned in section 7.2.4.
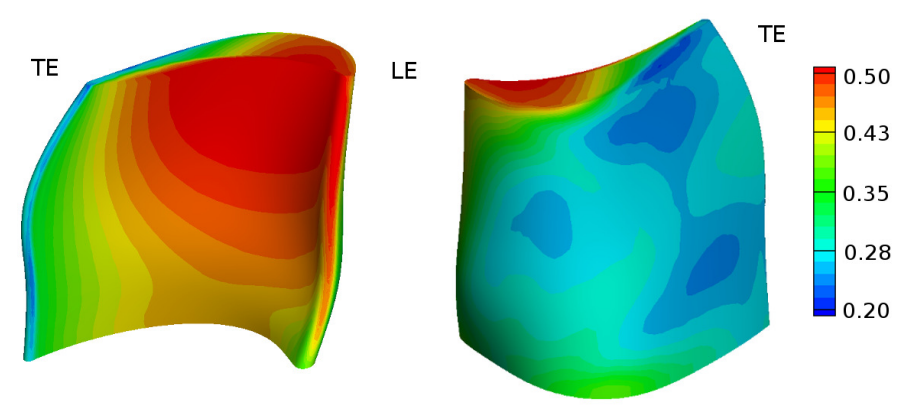

Figure 8.16: Time-averaged pressure contours on the blade surfaces, normalized by $P_{o 1}$.

\subsection{Rotor Inlet and Outlet Properties}

Contours of the time-averaged relative total enthalpy at the rotor inlet are shown in Fig. 8.17 for the two configurations. In both cases, the enthalpy peaked at approximately $50-70 \%$ of the radial span but at distinctly different circumferential locations relative to the blades. In addition to the main peaks, local maxima and minima were also observed. The non-uniformity of enthalpy distribution was generally lower in the VI case. Pitchwise spreading of the hot streaks was evident in both case, but it was much stronger in the $M P$ case.

Figure 8.18 shows time-averaged contours of relative total enthalpy at the rotor exit. The hot streaks remained distinct, although they had migrated toward the rotor casing from their spanwise locations at the rotor inlet. As at the rotor inlet, the enthalpy non-uniformity was lower in the $V I$ configuration. In the $M P$ case, radial diffusion of the 


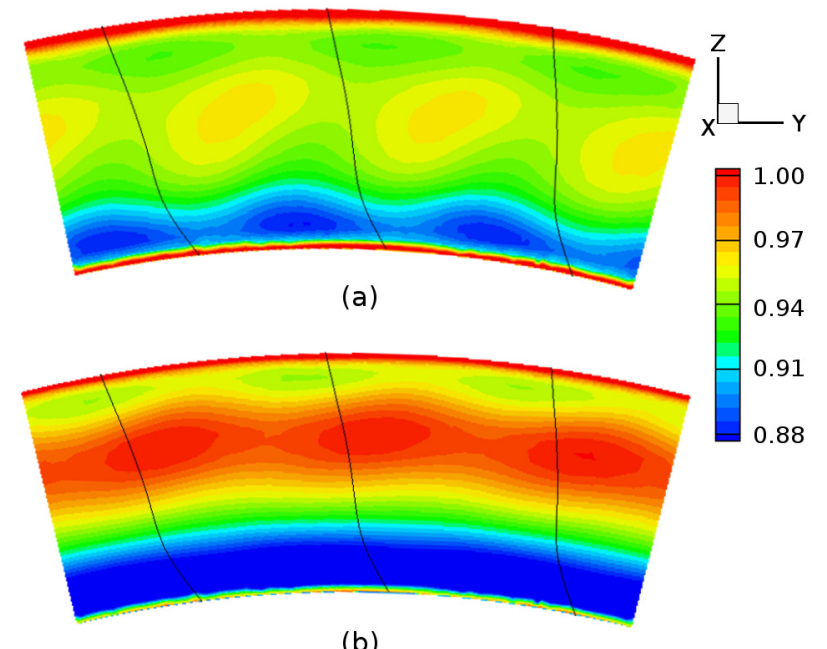

(b)

Figure 8.17: Time-averaged relative total enthalpy variation at rotor inlet for the (a) $V I$ and (b) $M P$ cases, normalized by $h_{o 1}$. The view is from downstream of the rotor, with dark lines indicating blade leading edge locations.

hot streaks was not very pronounced, but pitchwise diffusion was evident.

The fluctuations of relative total enthalpy at the rotor inlet are shown in Fig. 8.19.

The fluctuations were stronger in the VI case, particularly in the lower half of the blade span, where they were largely generated by the periodic passing of cooler fluid.

Figure 8.20 shows the fluctuations of relative total enthalpy at the exit of the rotor. In general, these fluctuations were weaker at the rotor exit than at the inlet (Fig. 8.19). This is true for both cases, but changes in the $V I$ case were larger, largely due to the significant reduction in hot streak strength (Fig. 8.3). In the $V I$ case, regions of high enthalpy had relatively weak enthalpy fluctuations. In contrast, both the enthalpy and its fluctuations in the $M P$ case were high at approximately the same spanwise position, which would have adverse effects on subsequent turbine stages.

Table 8.1 presents mass-averaged flow properties at the rotor outlet for the three 


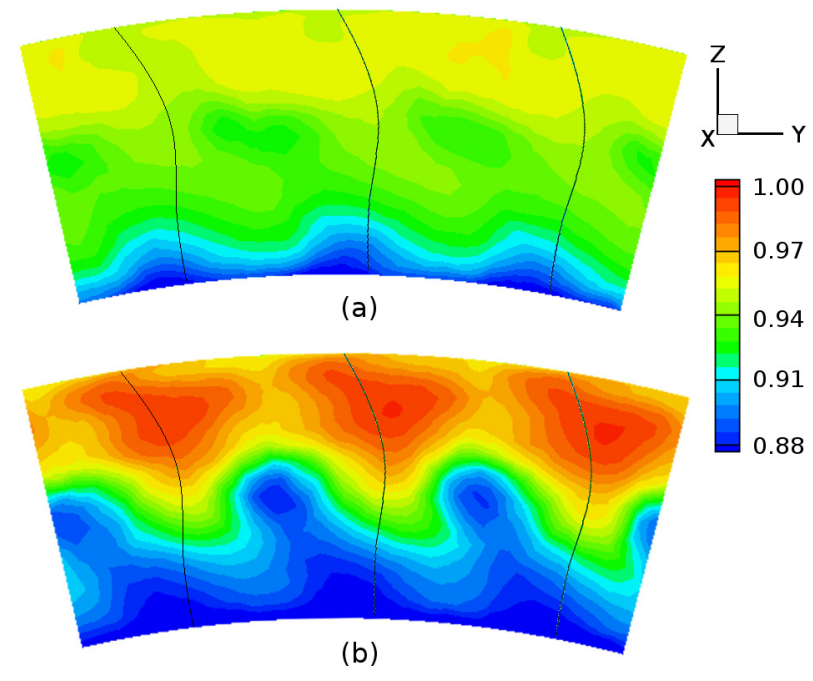

Figure 8.18: Time-averaged relative total enthalpy variation at rotor exit for the (a) $V I$ and (b) $M P$ cases, normalized by $h_{o 1}$. The view is from downstream of the rotor, with dark lines indicating blade trailing edge locations.

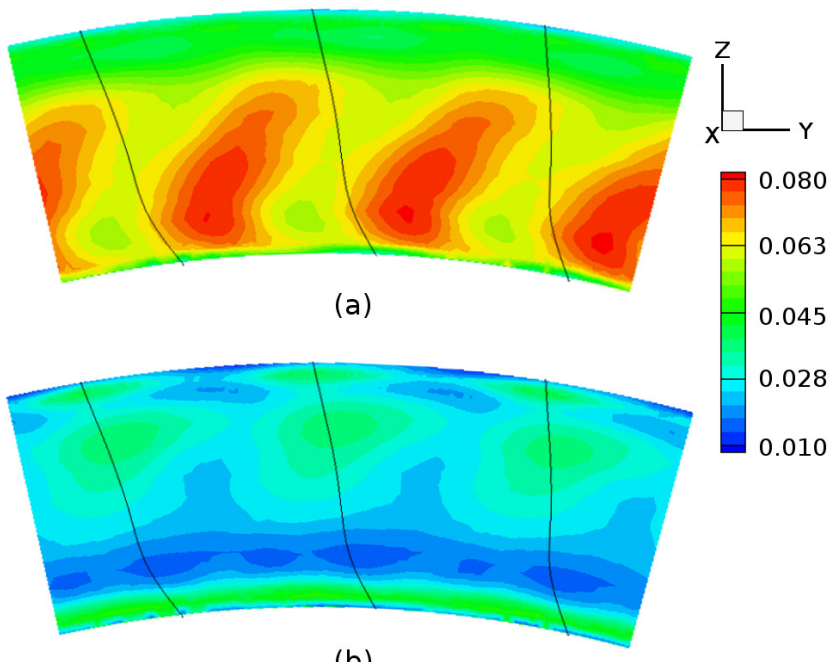

(b)

Figure 8.19: Standard deviation of relative total enthalpy at rotor inlet for the (a) VI and (b) $M P$ cases, normalized by $h_{o 1}$. The view is from downstream, with black lines indicating blade leading edge locations. 


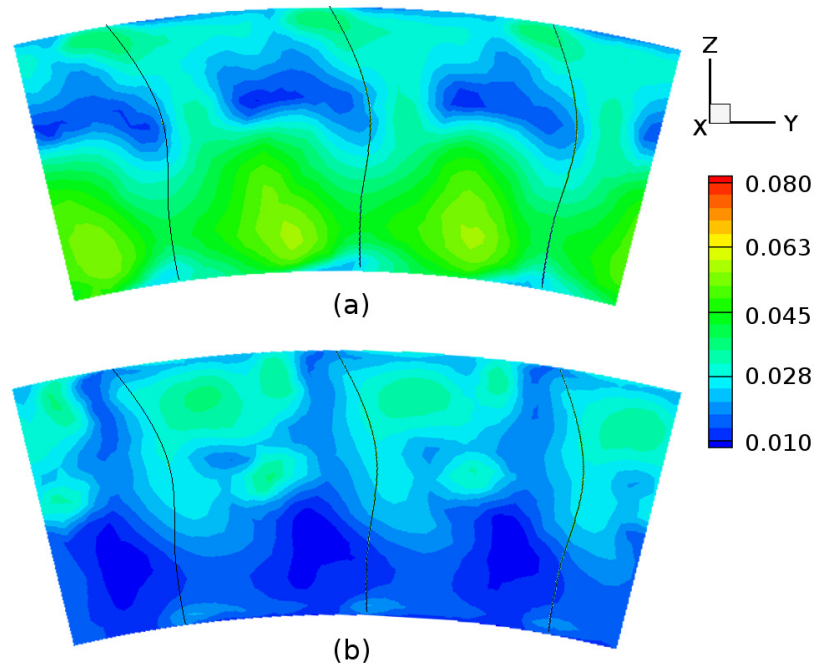

(b)

Figure 8.20: Standard deviation of relative total enthalpy at rotor exit for the (a) VI and (b) $M P$ cases, normalized by $h_{o 1}$. The view is from downstream of the rotor, with dark lines indicating blade trailing edge locations.

\begin{tabular}{|c|c|c|c|}
\hline Hot Streak Configuration & $h_{3} / h_{o 1}$ & $h_{o 3} / h_{o 1}$ & $P_{o 3} / P_{o 1}$ \\
\hline \hline Vane-impinging & 0.7331 & 0.7561 & 0.3197 \\
\hline Mid-pitch & 0.7313 & 0.7561 & 0.3195 \\
\hline Reference case & 0.7210 & 0.7440 & 0.3209 \\
\hline
\end{tabular}

Table 8.1: Mass-averaged values at the rotor exit, normalized by the corresponding massaveraged inlet value.

configurations. The differences in static enthalpy and total enthalpy were less than $0.3 \%$ between the two hot streak cases, and about $1.5 \%$ between them and the reference case. Differences in total pressure were much lower. This agrees with the finding of An et al. (2009) that hot streaks have a very small effect on the time-averaged conditions at the outlet. Note that the static enthalpy comprises more than $95 \%$ of the total enthalpy. Changes in fluid flow angle (not shown here) were found to be negligible, as were differences in the velocity and pressure distributions. 


\subsection{Spatially-Averaged Properties}

The circumferentially-averaged relative total enthalpy over the rotor domain inlet is shown in Fig. 8.21. The difference in overall average value between the two cases is less than $0.05 \%$. Although the shapes of these profiles are qualitatively similar, the enthalpy in the $M P$ case increases more toward the rotor casing than that in the $V I$ case. This is due to the hot streak spreading to a greater extent in the $V I$ case, lowering the enthalpy near the rotor casing and raising the enthalpy near the hub. The lack of spreading in the $M P$ case results in more high-enthalpy fluid near the casing, and vice-versa.

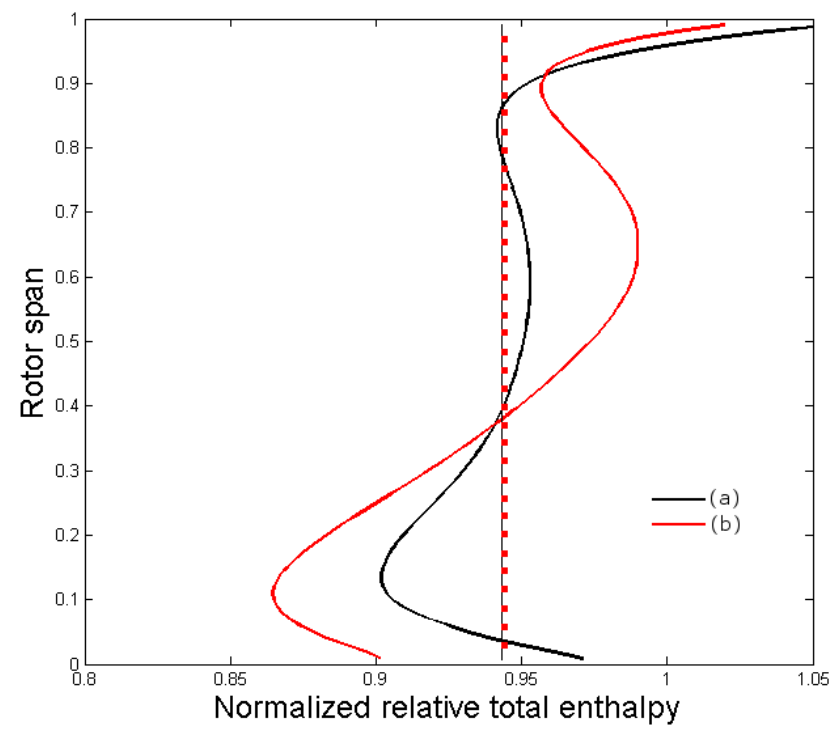

Figure 8.21: Relative total enthalpy, circumferentially averaged across the inlet of the rotor domain for the (a) $V I$ and (b) $M P$ case, normalized by $h_{o 1}$. The dashed/dotted lines indicate spanwise averages.

Figure 8.22 shows the circumferentially-averaged profile of relative total enthalpy on the leading edge surface of the rotor blades. The overall average is approximately $2 \%$ 
higher in the $V I$ case, though the peak is higher in the $M P$ case. The decreased average for the $M P$ case may be due to the increase in segregation effect, as discussed in Section 8.1, as fluid of high enthalpy tends to move past the leading edge, while the hot fluid in the $V I$ case tends to impact on the leading edge. The peak of the profile in the $M P$ case is shifted toward the blade tip, with a large portion of low enthalpy near the rotor hub. This is consistent with Fig. 8.21, and due to the reduced spreading of the hot streak in the $M P$ case.

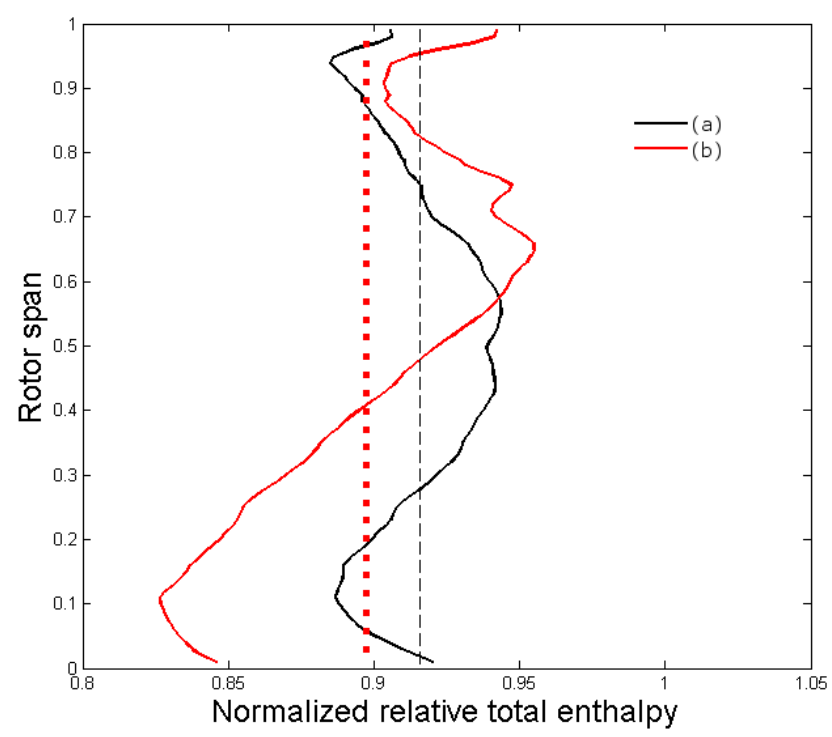

Figure 8.22: Relative total enthalpy, circumferentially averaged over the leading edge surface of the rotor domain for the (a) $V I$ and (b) $M P$ case, normalized by $h_{o 1}$. The dashed/dotted lines indicate spanwise averages.

Table 8.2 lists the geometric averages of relative total enthalpy for each of the blade surfaces (as shown in Fig. 8.23), as well as the average over the entire surface of the blade. Although the overall averages have comparable values (within 0.5\%), the averages on each surface differ by up to $5 \%$. This is indicative of the large variation in enthalpy 
distribution over the surface of the blades.

Table 8.2: Geometric average of normalized relative total enthalpy for blade surfaces.

\begin{tabular}{|c|c|c|}
\hline Surface & $\boldsymbol{V I}$ Case & $\boldsymbol{M P}$ Case \\
\hline \hline Leading Edge & 0.922 & 0.900 \\
\hline Suction Side & 0.903 & 0.883 \\
\hline Pressure Side & 0.935 & 0.966 \\
\hline Trailing Edge & 0.911 & 0.915 \\
\hline Tip & 0.909 & 0.951 \\
\hline Total Blade & 0.916 & 0.919 \\
\hline
\end{tabular}

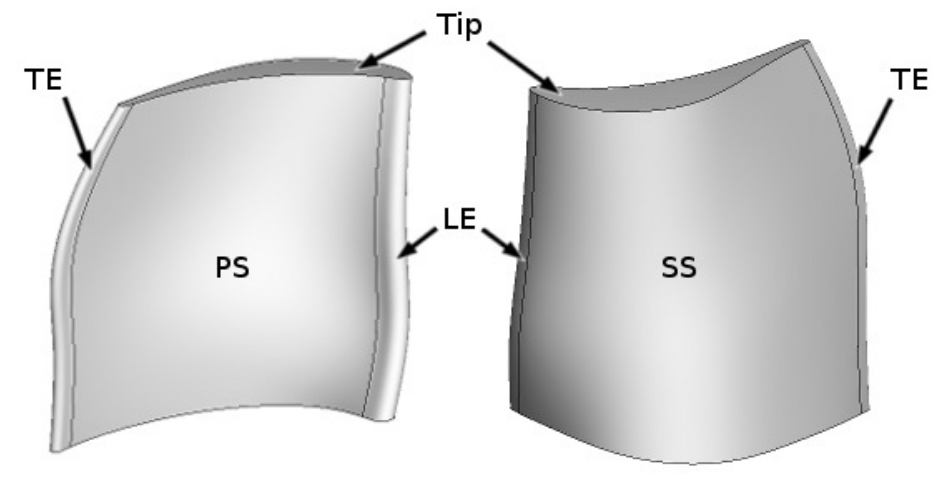

Figure 8.23: Designation of rotor blade surface geometry.

\subsection{Turbine Efficiency}

Because the specific heat ratio $\gamma$ depends on temperature, the isentropic efficiency of the turbine cannot be defined in a unique manner. For comparative purposes, we defined the total-to-total isentropic efficiency as

$$
\eta_{t t i s}=\frac{h_{01}-h_{03}}{h_{01}\left[1-\left(\frac{\mathbf{C p}_{3}}{\mathbf{C p}_{1}}\right)\left(\frac{P_{03}}{P_{01}}\right)^{\left(\frac{\gamma-1}{\gamma}\right)}\right]}
$$


where $\gamma$ was calculated as the average of the mass-weighted values at the stator inlet and the rotor outlet Zucker and Biblarz (2002). By comparison to its value for the reference configuration, the isentropic efficiency was found to be $3.8 \%$ lower for the $V I$ case and $3.3 \%$ lower for the $M P$ case.

An alternative definition of efficiency is the total-to-total efficiency defined by Denton (1993) and Pullan et al. (2006) as

$$
\eta_{t t}=\frac{h_{01}-h_{03}}{\left(h_{01}-h_{03}\right)+T_{03}\left(s_{3}-s_{1}\right)}
$$

A third definition of efficiency, although rarely used, is the rational efficiency discussed by Young and Horlock (2006), which has a form similar to Eq. 8.2 but with the dead-state temperature instead of $T_{03}$. By comparison to the reference case of uniform inlet total temperature, the total-to-total efficiency was found to be $5.2 \%$ lower for the $V I$ case and $4.7 \%$ lower for the $M P$ case. This result is qualitatively consistent with the relative values of the isentropic efficiencies. Both set of results suggest that the presence of hot streaks, irrespectively of their circumferential locations, reduces the turbine's efficiency. It is interesting to note that although Eq. 8.2 takes into account entropy changes due to the mixing of two streams of different total temperature (Denton (1993)) and Eq. 8.1 does not, both lead to the same conclusion for the relative effect of hot streaks. 


\section{Chapter 9}

\section{Conclusions and Recommendations}

\section{for Future Work}

\subsection{Conclusions}

In order to evaluate the performance effects of non-uniform inlet temperature conditions, two contrasting models with realistic total temperature distributions have been devised and applied to the inlet boundary of a simulated high-pressure turbine stage. These models correspond to the common configurations of hot streaks impinging on the stator vane leading edges (vane-impinging, $V I$ ) and of hot streaks passing between stator vanes (midpitch, MP). These distributions only differ from each other by an azimuthal offset, and are derived from experimental data of the time-averaged flow between a reverse-flow combustor and a transonic, high-pressure, axial turbine.

Unsteady numerical simulations have been performed, with the objective of com- 
paring the results of these two cases to one another and to a reference case having uniform inlet conditions. The emphasis of this comparison was on thermal effects and overall performance, as they are the most direct results of inlet temperature non-uniformity.

In the $V I$ configuration, the hot streaks produced a significantly higher timeaveraged heat load on the vanes and a lower heat load on the blades, compared to the $M P$ configuration, in which the highest heat load was on the surface of the blades.

As the vane-impacting hot streaks impinged on the stator vanes, they also spread in the spanwise direction under the influence of the casing passage vortices and the radial pressure gradient; this resulted in a stream entering the rotor with relatively low temperature variations. The mid-pitch hot streaks were convected undisturbed past the relatively cool vane section, imposing on the rotor inlet a temperature field with temporal and spatial fluctuations of much higher amplitude.

Relatively high time-averaged values of enthalpy were found on the pressure side of the blades in the $M P$ configuration. The non-uniformity of the time-averaged enthalpy on the blade surfaces was lower in the $V I$ configuration. The flow exiting the rotor section was much less non-uniform in the $V I$ case, primarily due to the low fluctuations at the rotor inlet. However, differences in calculated efficiency were not found to be significant between the two configurations.

The fluctuations in heat loading on the vane and blade surfaces were found to be comparable for the two configurations. Fluctuations in the fluid at the inlet and the exit of the rotor section were slightly higher in the VI case, as the hot streaks spread spanwise.

The orientation of the hot streaks had no significant effect on the flow angle, 
velocity, or pressure loading on the vanes and blades; these parameters were nearly identical in all studied cases.

The difference in heating of the rotor blades emphasizes that not only the existence, but also the azimuthal positioning and spacing of hot streaks need to be taken into account in gas turbine analysis and design. In general, the much stronger localization of enthalpy exiting the rotor for the $M P$ case would likely result in more intense off-design performance of the subsequent low-pressure turbine.

\subsection{Recommendations for Future Work}

As the time and resources allocated to this study were limited, only two hot streak configurations were studied. An additional non-uniform inlet temperature field was proposed by $\mathrm{P} \& \mathrm{WC}$, the simulation of which is under way at the time of writing. This field consists of the original radial profile applied across the entire inlet boundary without circumferential variation. The results of this simulation are beyond the scope of this study, as the simplified field is less realistic than the $V I$ and $M P$ cases.

It would have been desirable to study additional cases with different alignments between the hot streaks and stator vanes. More realistic cases involving non-reducing ratios of streaks-to-vanes and of vanes-to-blades would also be of interest, though they would entail simulations of the entire turbine, as there would not be any azimuthal periodicity. In the case of the studied turbine, the computational domain would increase by a factor of 13, producing a grid consisting of approximately 22.1 million cells; this would require much

more time and resources than is practically available, though it may be somewhat more 
representative of current engines.

A more useful and realizable recommendation would be to conduct a study similar to the completed one, though including analysis of the cooling system, primarily in the rotor section. The current interpretation of the present results is based on the assumption that increased cooling capacity in the rotor section is more expensive than in the stator section, in terms of initial design and manufacturing, as well as performance-reducing operating losses. Although this view is common in gas turbine design and analysis, a more detailed simulation would allow for the quantification of the requirements and potential losses associated with modification of the cooling system. The resources required for this supplemented simulation would be somewhat increased, but the primary hindrance may be in obtaining specifications for the cooling system and accurately implementing them in the numerical model. An incremental improvement may be the inclusion of more realistic model of the axial gap region between the stationary and rotating sections, such as a stepped gap and coolant injection.

This study has mainly focused on the evaluation of thermal effects and overall performance, but the scope of analysis of the results could be extended. For example, a detailed analysis of the segregation effect may illuminate slight differences in coherent structure evolution and convection that were not observed in the current study that only examined general trends.

The addition of subsequent turbine stages would allow for the analysis of the effects of the different previously-mentioned levels of rotor exit non-uniformity. While periodic non-uniformities of higher amplitude were reasoned to be detrimental to the performance 
downstream stages, conclusive results would require additional simulations. This case could be composed either by adding a second stage to the current geometry, or by implementing the time-periodic exit field as the inlet condition in a separate computational domain. 


\section{References}

Adamczyk, J. J. (2000). Aerodynamic analysis of multistage turbomachinery flows in support of aerodynamic design. Journal of Turbomachinery 122(2), 189-217.

AIAA (1998). Guide for the verification and validation of computational fluid dynamics simulations. AIAA Journal (G-077-1998).

Alkabie, H. (2000). Design methods of the ABB ALSTOM POWER gas turbine dry low emission combustion system. Proceedings of the Institution of Mechanical Engineers, Part A: Journal of Power and Energy 214(4), 293 - 315.

An, B.-T., J.-J. Liu, and H.-D. Jiang (2009). Numerical investigation on unsteady effects of hot streak on flow and heat transfer in a turbine stage. Journal of Turbomachinery $131(3), 031015$.

Ansys (2006a). FLUENT 6.3 User's Guide. Ansys Inc.

Ansys (2006b). GAMBIT 2.3 Documentation. Ansys Inc.

Bardina, J., P. Huang, and T. Coakley (1997). Turbulence modeling validation. AIAA Journal, 1-16.

Barth, T. and D. Jespersen (1989). The design and application of upwind schemes on 
unstructured meshes. Technical Report AIAA-89-0366, AIAA 27th Aerospace Science Meeting, Reno, Nevada.

Blazek, J. (2005). Computational Fluid Dynamics: Principles and Applications (2nd ed.). Elsevier Boston.

Boussinesq, J. (1877). Essai sur la theorie des eaux courantes, memoires presentes par divers savants. l'Académie des Sciences 23(24), Paris, France, 1-680.

Bradshaw, P. (1987). Turbulent secondary flows. Annual Review of Fluid Mechanics 19, 53-74.

Bradshaw, P. (1997). Understanding and prediction of turbulent flow - 1996. International Journal of Heat and Fluid Flow 18, 45-54.

Butler, T., O. Sharma, H. Joslyn, and R. Dring (1989). Redistribution of an inlet temperature distortion in an axial flow turbine stage. Journal of Propulsion and Power 5, 64-71.

Casciaro, C., M. Treiber, and M. Sell (2000). Unsteady transport mechanisms in an axial turbine. Journal of Turbomachinery 122, 604-612.

Celik, I. and J. Li (2005). Assesment of numerical uncertainty for the calculations of turbulent flow over a backward-facing step. International Journal of Numerical Methods in Fluids 49, 1015-1031.

Chakraborty, P., S. Balachandar, and R. Adrian (2005). On the relationships between local vortex identification schemes. Journal of Fluid Mechanics 535, 189 - 214.

Chang, D. and S. Tavoularis (2007). Numerical simulation of turbulent flow in a 37-rod 
bundle. Nuclear Engineering and Design 237, 575-590.

Chang, D. and S. Tavoularis (2009a). Parallel computations of unsteady threedimensional flows in a high pressure turbine. In Lecture Notes in Computer Science, Berlin, pp. 20-29. High Performance Computing Symposium: Springer-Verlag.

Chang, D. and S. Tavoularis (2009b). Unsteady vortices and blade loading in a highpressure turbine. In ASME Turbo Expo, Number GT2009-59189, Orlando, USA.

Clark, J., G. Stetson, S. Magge, R. Ni, C. Haldeman Jr., and M. Dunn (2000). The effect of airfoil scaling on the predicted unsteady loading on the blade of a 1 and $1 / 2$ stage transonic turbine and a comparison with experimental results. In ASME Turbo Expo, Number 2000-GT-0446, Munich, Germany. ASME.

Clark, J. P. and E. A. Grover (2007). Assessing convergence in predictions of periodicunsteady flowfields. Journal of Turbomachinery 129(4), 740 - 749 .

Denton, J. (1993, October). Loss mechanisms in turbomachines. Journal of Turbomachinery 115, 621-656.

Denton, J. and L. Xu (1989). The trailing edge loss of transonic turbine blades. ASME Paper (89-GT-278).

Dorney, D. J. and R. L. Davis (1993). Numerical simulation of turbine 'hot spot' alleviation using film cooling. Journal of Propulsion and Power 9(3), 329 - 336.

Dorney, D. J., R. L. Davis, D. E. Edwards, and N. K. Madavan (1992). Unsteady analysis of hot streak migration in a turbine stage. Journal of Propulsion and Power 8(2), 520 $-529$. 
Dorney, D. J. and K. Gundy-Burlet (1996). Hot-streak clocking effects in a 1-1/2 stage turbine. Journal of Propulsion and Power 12(3), 619 - 620.

Dubief, Y. and F. Delcayre (2000). On coherent-vortex identification in turbulence. Journal of Turbulence $1(1468-5248)$.

Faragher, J. (2004). Probabilistic methods for the quantification of uncertainty and error in computational fluid dynamics simulations. Technical Report DSTO-TR-1633, Australian Government Department of Defence.

Goldstein, R. and R. Spores (1988). Turbulent transport on the endwall in the region between adjacent turbine blades. ASME Journal of Heat Transfer 110, 862-869.

Gundy-Burlet, K. L. and D. J. Dorney (1997). Influence of 3D hot streaks on turbine heat transfer (97-GT-422). International Journal of Turbo and Jet Engines 14(3), $123-132$.

Gundy-Burlet, K. L. and D. J. Dorney (2000). Effects of radial location on the migration of hot streaks in a turbine. Journal of Propulsion and Power 16(3), 377 - 387.

Han, J.-C. (2004). Recent studies in turbine blade cooling. International Journal of Rotating Machinery 10(6), 443-457.

Hawthorne, W. R. (1951). Secondary circulation in fluid flow. Proceedings of the Royal Society of London, Series A (Mathematical and Physical Sciences) 206, 374-387.

He, L., V. Menshikova, and B. Haller (2004). Influence of hot streak circumferential length-scale in transonic turbine stage. In ASME Turbo Expo, Number GT2004-53370, Vienna, Austria, pp. $1117-1126$. 
He, L., V. Menshikova, and B. Haller (2007). Effect of hot-streak counts on turbine blade heat load and forcing. Journal of Propulsion and Power 23(6), 1235 - 1241.

Hinze, J. (1975). Turbulence. McGraw-Hill Book Company, New York.

Hunt, J., C. Wray, and P. Moin (1988). Eddies, streams, and convergence zones in turbulent flows. Technical Report CTR-S88, Center for Turbulence Research, Stanford University.

Jeong, J. and F. Hussain (1995). On the identification of a vortex. Journal of Fluid Mechanics 285, $69-94$.

Joslyn, H. D. and R. P. Dring (1989). Three dimensional flow and temperature profile attenuation in an axial flow turbine. Report AFOSRTR-89-0439, AFOSR.

Lakshminarayana, B. (1996). Fluid Dynamics and Heat Transfer of Turbomachinery. John Wiley and Sons New York.

Langston, L. (1980). Crossflows in a turbine cascade passage. ASME Journal of Engineering for Power 102, 866-874.

Lastiwka, D. (2008). Influence of rotor blade scaling on the numerical simulation of a high pressure gas turbine. Master's thesis, University of Ottawa.

Launder, B. and D. Spalding (1974). The numerical computation of turbulent flows. Computer Methods in Applied Mechanics and Engineering 3(2), 269-289.

Li, L., X. Peng, and T. Liu (2006). Combustion and cooling performance in an aero-engine annular combustor. Applied Thermal Engineering 26(16), 1771 - 1779.

Mårtensson, H., B. Laumert, and T. Fransson (2003). Aeromechanical aspects on un- 
steady flow in turbines. In 33rd AIAA Fluid Dynamics Conference and Exhibit, Number AIAA-2003-3997, Orlando, Florida.

Mathison, R., C. Haldeman, and M. Dunn (2010). Aerrodynamics and heat transfer for a cooled one and one-half stage high-pressure turbine-Part II: Influence of inlet temperature profile on blade row and shroud. In ASME Turbo Expo, Number GT201022718, Glasgow, UK.

Mehta, U. (1996). Guide to credible computer simulations of fluid flows. AIAA Journal 12(5), 940-948.

Menter, F. (1991). Influence of freestream values on $\mathrm{k}-\omega$ turbulence model predictions. AIAA Journal 30(6), 1657-1659.

Menter, F. (1992). Performance of popular turbulence models for attached and separated adverse pressure gradient flows. AIAA Journal 30(8), 2066-2072.

Menter, F. (1994). Two-equation eddy-viscosity turbulence models for engineering applications. AIAA Journal 32(8), 1598-1605.

Menter, F. (2002). CFD best practice guidelines for CFD code validation for reactorsafety applications. Technical Report FIKS-CT-2001-00154, Evaluation of Computational Fluid Dynamic Methods for Reactor Safety Analysis Project (ECORA Project).

Miller, R., R. Moss, R. Ainsworth, and N. Harvey (2003). Wake, shock, and potential field interactions in a 1.5 stage turbine-Part I: Vane-rotor and rotor-vane interaction. Journal of Turbomachinery 125, 33-39.

Moore, J. and R. Y. Adhye (1985). Secondary flows and losses downstream of a turbine 
cascade. Journal of Engineering for Gas Turbines and Power 107(4), 961 - 968.

Munk, M. and R. C. Prim (1947). On the multiplicity of steady gas flows having the same streamline pattern. Proceedings of the National Academy of Sciences, U.S.A. 33, 137141.

Niestroj, O. and P. Came (1998). Three-dimensional flow predictions in axial-flow turbine cascades. In ASME Paper, Number 98-GT-325, Fairfield, NJ, USA.

Payne, S. (2001). Unsteady Loss in a High Pressure Turbine Stage. Ph. D. thesis, University of Oxford.

Povey, T., K. Chana, and T. Jones (2003). Heat transfer measurements on an intermediate-pressure nozzle guide vane tested in a rotating annular turbine facility, and the modifying effect of a non-uniform inlet temperature profile. Proceedings of the Institution of Mechanical Engineers, Part A: Journal of Power and Energy 217(4), $421-432$.

Povey, T., K. S. Chana, T. V. Jones, and J. Hurrion (2007). The effect of hot-streaks on HP vane surface and endwall heat transfer: An experimental and numerical study. Journal of Turbomachinery 129(1), 32-43.

Prasad, D. and G. J. Hendricks (2000). A numerical study of secondary flow in axial turbines with application to radial transport of hot streaks. Journal of Turbomachinery 122, $667-673$.

Pullan, G. (2006). Secondary flows and loss caused by blade row interaction in a turbine stage. Journal of Turbomachinery 128(3), 484-491. 
Pullan, G., J. Denton, and E. Curtis (2006). Improving the performance of a turbine with low aspect ratio stators by aft-loading. Journal of Turbomachinery 128(3), 492-499.

Qingjun, Z., W. Huishe, Z. Xiaolu, and X. Jianzhong (2007). Numerical investigation on the influence of hot streak temperature ratio in a high-pressure stage of vaneless counter-rotating turbine. International Journal of Rotating Machinery (56097).

Qingjun, Z., D. Jianyi, W. Huishe, Z. Xiaolu, and X. Jianzhong (2010). Tip clearance effects on inlet hot streak migration characteristics in high pressure stage of a vaneless counter-rotating turbine. Journal of Turbomachinery 132(011005).

Rai, M. (1987). Navier-Stokes simulations of rotor/stator interaction using patched and overlaid grids. Journal of Propulsion and Power 3(5), 387-396.

Rai, M. and N. Madavan (1990). Multi-airfoil Navier-Stokes simulations of turbine rotorstator interaction. Journal of Turbomachinery 112, 377-384.

Roache, P. (1998). Verification and Validation in Computational Science and Engineering. Hermosa Publishers, Albuquerque, New Mexico, USA.

Roulund, A., B. Mutlu Sumer, J. Fredsoe, and J. Michelsen (2005). Numerical and experimental investigation of flow and scour around a circular pile. Journal of Fluid Mechanics 534, 351-401.

Schaub, U., E. Vlasic, and S. Moustapha (1993). Effect of tip clearance on the performance of a highly loaded turbine stage. AGARD-CP-537 Paper 29, Technology Requirements for Small Gas Turbines.

Schobeiri, M. (2005). Turbomachinery Flow Physics and Dynamic Performance. Berlin: 
Spinger-Verlag.

Shang, T. and A. Epstein (1997). Analysis of hot streak effects on turbine rotor heat load. Journal of Turbomachinery 119(3), 544-553.

Sharma, O. and T. Butler (1987). Predictions of endwall losses and secondary flows in axial flow turbine cascades. Journal of Turbomachinery 109, 229-236.

Shih, T.-H., W. Liou, A. Shabbir, Z. Yang, and J. Zhu (1994). A new k-e eddy viscosity model for high reynolds number turbulent flows-model developement and validation. Technical Report NASA TM-106721, Institue for Computational Mechanics in Propulsion and Center for Modeling of Turbulence and Transition, Lewis Research Center.

Sondak, D. L. and D. J. Dorney (2000). Simulation of coupled unsteady flow and heat conduction in turbine stage. Journal of Propulsion and Power 16(6), 1141 - 1148.

Sondak, D. L., V. Gupta, P. D. Orkwis, and D. J. Dorney (2002). Effects of blade count on linearized and nonlinear hot streak clocking simulations. Journal of Propulsion and Power 18(6), $1273-1279$.

Spalart, S. and S. Allmaras (1992). A one-equation turbulence model for aerodynamic flows. In AIAA Paper, Number 92-0439, pp. 5-21.

Speziale, C. (1996). Modeling of Turbulent Transport Equations, Simulation and Modeling of Turbulent Flow. Oxford University Press, New York.

Takeishi, K., M. Matsuura, S. Aoki, and T. Sato (1990). An experimental study of heat transfer and film cooling on low aspect ratio turbine nozzles. Journal of Turbomachinery 112, 488-496. 
Turrell, M. D., P. J. Stopford, K. J. Syed, and E. Buchanan (2004). CFD simulation of the flow within and downstream of a high-swirl lean premixed gas turbine combustor. In ASME Turbo Expo, Number GT2004-53112, Vienna, Austria, pp. 31 - 38.

Van Wylen, G. and R. Sonntag (1978). Fundamentals of Classical Thermodynamics (2nd ed.). John Wiley and Sons New York.

Wilcox, D. (2000). Turbulence Modeling for CFD (2nd ed.). DCW Industries.

Xun, Z., H. Wanjin, and L. Zhiqiang (2005). Experimental investigation of energy loss in straight and bowed cascades with aft-loaded profiles. Journal of Aerospace and Power 19, 118-125.

Yakhot, V. and S. Orszag (1986). Renormalization group analysis of turbulence. 1: Basic theory. Journal of Scientific Computing 1(1), 3-51.

Young, J. and J. H. Horlock (2006). Defining the efficiency of a cooled turbine. Journal of Turbomachinery 128(4), $658-667$.

Zhang, S. and D. Choudhury (2006). Eigen helicity density: A new vortex identification scheme and its application in accelerated inhomogeneous flows. Physics of Fluids $18(5), 058104$.

Zhang, S., S. Mereu, J. Yan, and D. Choudhury (2007). Extraction of vortical flow features in a turbomachinery simulation. In 45th AIAA Aerospace Sciences Meeting and Exhibit, Number AIAA-2007-1285, Reno, Nevada.

Zucker, R. D. and O. Biblarz (2002). Fundamentals of Gas Dynamics (2nd ed.). John Wiley \& Sons, Inc. 